\title{
DISSERTATION
}

\section{TESTING EDDY COMPENSATION AND EDDY SATURATION \\ IN THE SOUTHERN OCEAN}

\author{
Submitted by \\ Daniel C. Jones \\ Department of Atmospheric Science
}

\author{
In partial fulfillment of the requirements \\ For the Degree of Doctor of Philosophy \\ Colorado State University \\ Fort Collins, Colorado \\ Spring 2013
}

\section{Doctoral Committee:}

Advisor: Takamitsu Ito

Thomas Birner

Nicole Lovenduski

David Randall

Simon Tavener 
Copyright by Daniel C. Jones 2013

All Rights Reserved 


\section{ABSTRACT \\ TESTING EDDY COMPENSATION AND EDDY SATURATION IN THE SOUTHERN OCEAN}

The Southern Ocean (SO) is a unique and dynamic component of the climate system. Due in part to its cold temperatures and large surface area, the SO is an important region for the transfer of heat, momentum, and climatically relevant gases between the atmosphere and the interior ocean. The strong westerly winds above the SO help drive a powerful current (i.e. the Antarctic Circumpolar Current or ACC) that connects Earth's ocean basins in a global overturning circulation. In recent decades, these winds have strengthened and shifted poleward. Despite this change in surface forcing, no clear observational signal of the oceanic density structure's response has yet been detected. The eddy compensation hypothesis posits that changes in the direct wind-driven overturning circulation are balanced by changes in the eddy-induced meridional circulation, effectively rendering SO stratification insensitive to wind stress. The closely related (but not identical) eddy saturation hypothesis suggests that the ACC is also insensitive to increased wind stress, since additional energy ends up in the mesoscale eddy field instead of in the zonal mean circulation. In this work, we examine the viability of the eddy compensation and saturation hypotheses on interannual, decadal, and centennial timescales.

Using a combination of theory and idealized numerical simulations, we show that it may take the Southern Ocean many decades to centuries to fully equilibrate with the world ocean following a change in wind stress. As such, it may be difficult to detect changes in isopycnal slope using the few decades of available observational data. We also explore the characteristics of eddy-driven interannual variability and examine how this variability may affect the decadal-scale adjustment of the global ocean. Our results suggest that departures from the eddy compensation regime may be important on decadal and centennial timescales,

on which the interaction between regional Southern Ocean circulation and global ocean 
circulation is significant. As such, we suggest that Southern Ocean overturning circulation is likely to strengthen in response to recent and future climate change, with implications for the global carbon cycle and climate. 


\section{ACKNOWLEDGMENTS}

First, let me say that it has been a pleasure working in Taka Ito's group for the past several years. I could not have asked for a better advisor. I also wish to express my gratitude towards my doctoral committee members for their efforts and insight. I thank Taka Ito for his astute guidance, tireless generosity, and contagious optimism, Thomas Birner for his knowledge, encouragement, and his willingness to share both, Nicole Lovenduski for her relentless enthusiasm and her awareness of the big picture, David Randall for his excellent experimental suggestions and thought-provoking questions, and Simon Tavener for his enlightening perspective.

This project was carried out at two different institutions, namely Colorado State Univer-

sity and Georgia Tech. Computational support was provided by Colorado State University, the NASA Advanced Supercomputing Division, and Georgia Tech's Parternship for an Advanced Computing Environment. I am grateful for support from the National Oceanic and Atmospheric Administration (NOAA grant NA08OAR4320893), the National Aeronautics and Space Administration (NASA grant NNX08AL72G), and the National Science Foundation. This project benefited greatly from discussions with other scientists, including (but not limited to) Ryan Abernathey, David Munday, Stephanie Downes, Andy Hogg, Anne Willem Omta, Baylor Fox-Kemper, Maxim Nikurashin, Yan Wu, ChuanLi Jiang, and Seong-Joong Kim. Thanks also goes to Jeremiah Sjoberg for help with formatting this document.

Finally, thank you to my family and friends for all of your support - especially to my wife Stephanie, for many years of love, encouragement, and for embracing our numerous interstate moves. I could not have finished this work without all of your support. 


\section{TABLE OF CONTENTS}

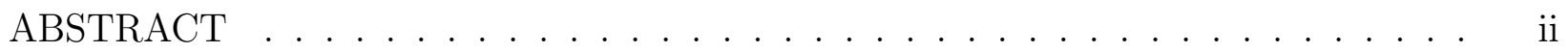

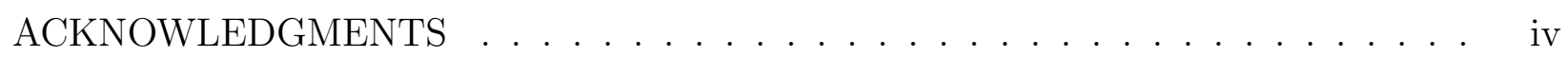

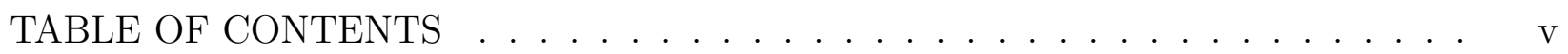

\section{CHAPTER}

1 Why study the Southern Ocean? . . . . . . . . . . . . . . . . . 1

1.1 Bathymetry and surface forcing . . . . . . . . . . . . 2

1.2 Large-scale circulation and global climate . . . . . . . . . . . 6

1.3 Southern Ocean dynamics . . . . . . . . . . . . . . . . . . . 10

1.4 Dissertation objectives and structure . . . . . . . . . . . . 18

2 How does the Southern Ocean interact with the Global Ocean? . . . . . . . 20

2.1 Observations of the global pycnocline . . . . . . . . . . . 20

2.2 A simple model of pycnocline structure . . . . . . . . . . . . . . 22

2.3 Equilibrium pycnocline depth . . . . . . . . . . . . . 25

2.4 Adjustment of the global pycnocline . . . . . . . . . . . . . . 28

2.5 Discussion . . . . . . . . . . . . . . . . . . . . . 37

3 Modeling philosophy and GCM description . . . . . . . . . . . 38

3.1 Aspects of climate modeling . . . . . . . . . . . . . . . 38

3.2 Primitive equation model description . . . . . . . . . . . . . . 42

3.3 Model bathymetry and surface forcing . . . . . . . . . . . . . 46

3.4 Step response experiments . . . . . . . . . . . . . . . 50

4 Coarse resolution step response experiments $\ldots \ldots \ldots \ldots 5$

4.1 Surface fluxes . . . . . . . . . . . . . . . . . . 55

4.2 Equilibrium response . . . . . . . . . . . . . . . . . . . 59

4.3 Transient response . . . . . . . . . . . . . . . . . . . . 72 
4.4 Weak wind and meridional ridge . . . . . . . . . . . . . . . . . 81

4.5 Discussion ........................... 82

5 Eddy permitting step response experiments . . . . . . . . . . . . . . . 85

5.1 Surface fluxes . . . . . . . . . . . . . . . . . . . 85

5.2 Quasi-equilibrium . . . . . . . . . . . . . . . 87

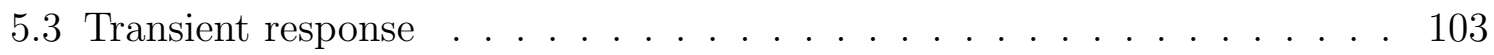

5.4 Interannual variability . . . . . . . . . . . . . . . . . 109

5.5 Discussion . . . . . . . . . . . . . . . . . . 116

6 Periodic forcing experiments . . . . . . . . . . . . . . . . . . 119

6.1 Wind stress magnitude . . . . . . . . . . . . . . . . 119

6.2 Mid-ocean seamount . . . . . . . . . . . . . . . . . . 128

7 Discussion and conclusions . . . . . . . . . . . . . . . . 132

7.1 Is the Southern Ocean insensitive to changing wind stress? . . . . . . . . 132

7.2 Modeling approach . . . . . . . . . . . . . . . 136

7.3 Future work . . . . . . . . . . . . . . . . . . . 138

REFERENCES . . . . . . . . . . . . . . . . . . . . . . . . 149 


\section{WHY STUDY THE SOUTHERN OCEAN?}

The ocean that surrounds Antarctica (i.e. the Southern Ocean) is a dynamic and important part of the climate system. The intense overlying winds transfer enormous amounts of energy into the ocean and encourage the exchange of gases (e.g. oxygen, nitrogen, carbon dioxide) between the air and sea. The absence of any meridional land barriers permits the existence of a powerful current that connects each of the ocean basins. This flow, called the Antarctic Circumpolar Current (ACC), establishes a global overturning circulation that transports heat, freshwater, momentum, chemical tracers, and other properties that may affect climate. In addition, the ACC is associated with steeply tilted surfaces of constant

density (isopycnals) that outcrop at high latitudes and establish a pathway between the atmosphere and the deep ocean. In this dissertation, we aim to better understand how the Southern Ocean adjusts to changes in wind forcing across a range of timescales.

In this chapter, we illustrate some of the factors that make the Southern Ocean an interesting region to study. No attempt is made at a complete review, but references to more detailed sources are placed throughout. For an excellent general overview of Southern Ocean dynamics, see Rintoul et al. (2001) and Thompson (2008). The bathymetry of the Southern Ocean and the dominant patterns of surface wind and buoyancy forcing are discussed in Section 1.1. The dynamics of the ACC and the associated local Southern Ocean meridional overturning circulation (SO-MOC) are examined in section 1.2. The sensitivity of SO mean circulation and variability to changing wind stress is discussed in section 1.3, in the context of the eddy compensation and eddy saturation hypotheses. Finally, the objectives and structure of this dissertation are discussed in section 1.4. 


\subsection{BATHYMETRY AND SURFACE FORCING}

The Southern Ocean is clearly demarcated at its southern end by the Antarctic continent. It has no obvious northern boundary; any attempt to distinguish the SO from the Atlantic, Pacific, and Indian basins to which it is connected will be somewhat arbitrary. For our purposes here, there is no need to specify a precise boundary between the SO and the rest of the ocean, though we should keep in mind that the dynamics of the SO can be quite different from the dynamics of Earth's other ocean basins.

\subsubsection{Bathymetry}

The geometry of the sea floor affects the paths of ocean currents throughout the water column (Gille et al., 2004). At depths where the current meets ridges and undersea mountains (i.e. seamounts), the current must obviously detour around such impenetrable features. In parts of the ocean with weak stratification (such as the cold waters of the Southern Ocean), currents tend to flow in a vertically coherent (i.e. barotropic) fashion due to Earth's rapid rotation and the ocean's small aspect ratio (i.e. its vertical scale is small compared to its horizontal scale). As such, bathymetry far below the surface can steer currents without coming into direct contact with them.

In a purely barotropic regime, the large-scale flow follows $f / H$ contours due to potential vorticity conservation, where $f$ is the planetary vorticity (i.e. the Coriolis parameter) and $H$ is a scale height, taken here to be the ocean depth. Float observations suggest this is a reasonable first-order model, since floats tend to spread along $f / H$ contours rather than across them (LaCasce, 2000). However, the SO is not perfectly barotropic, since it is at least weakly stratified and maintains a nonzero velocity shear due to thermal wind balance. Circumpolar flow in a stratified fluid constrained by rotation is often better described by an equivalent barotropic model, wherein the velocity decays exponentially with depth, as $v(z)=$ 
$v(0) \exp \left(-z / H_{0}\right)$, and the flow follows contours of $f / F_{0}$, where $F_{0}=H_{0}\left[1-\exp \left(-H / H_{0}\right)\right]$ (Killworth (1992), Marshall (1995b), Krupitsky et al. (1996)). Note that in the limit as $H_{0}$ approaches infinity, the equivalent barotropic model reduces to the simple barotropic case. Recent estimates suggest that the scale factor $H_{0}$ in the SO ranges between 500 and $1000 \mathrm{~m}$ (Gille (2003), Karsten and Marshall (2002)). In any case, the equivalent barotropic model helps us understand why the mean current does not always appear to avoid bathymetric features in a simple barotropic fashion.

The ocean floor around Antarctica exhibits considerable large-scale structure, as seen in Figure 1.1. Kilometer-scale changes in depth occur over a few hundred kilometers in the horizontal. The fronts of the ACC appear to bend around certain features, such as the Campbell Plateau near New Zealand and the ridges of the Scotia Arc just downstream of Drake Passage (Gordon et al. (1978), Gille et al. (2004)). The fronts are somewhat constrained by the bottom topography, in that they are not able to meander much in the meridional direction. Note that there are large patches over which the ocean depth does not change substantially, and in those regions the fronts of the ACC are relatively less constrained by the bathymetry and may meander more freely.

\subsubsection{Surface forcing}

Next we turn our attention to the dominant surface forcings in the SO that control the exchange of momentum and buoyancy with the atmosphere. Strong eastward winds encircle Antarctica, as seen in Figure 1.2 (left). These winds must, in a climatological mean sense, drive an equatorward Ekman flow in the surface layer that transports cold water away from the pole. This transport happens at all longitudes, but since the wind stress pattern lacks zonal symmetry, Ekman transport will exhibit some variability along a given longitude circle. Judging from the wind stress, Ekman transport is strongest in the Indian Sector of the Southern Ocean and weakest in the Eastern Pacific. 


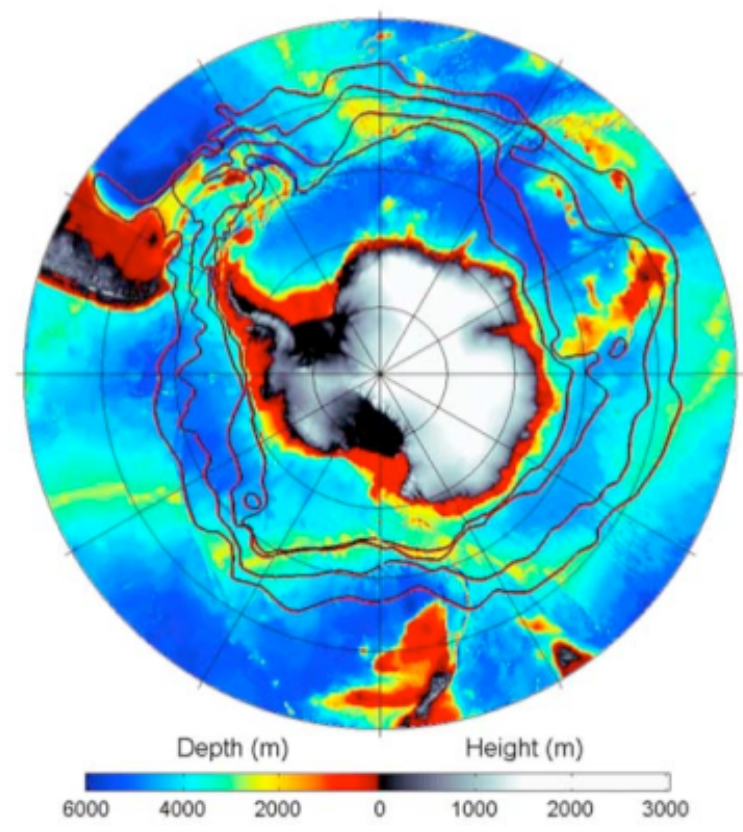

FIG. 1.1. The bathymetry and topography of Antarctica and the Southern Ocean, with several fronts of the Antarctic Circumpolar Current (solid lines). From (Mayewski et al., 2009).

The pattern of buoyancy forcing (Figure 1.2, right) is complex; it includes contributions from longwave and shortwave radiative fluxes, latent and sensible heat fluxes, as well as evaporation, precipitation, and freshwater runoff from land masses. Broadly speaking, the surface ocean loses buoyancy near Antarctica, gains buoyancy on the poleward flank of the ACC, and loses buoyancy on its equatorward flank. The strong meridional gradient in buoyancy flux helps to establish changes in stratification with latitude. As with wind stress, buoyancy flux exhibits considerable variation along longitude circles.

We should note that any given snapshot of the real ocean-atmosphere system bears little resemblance to a climatological mean picture like the one shown in Figure 1.2. Such a mean emerges after averaging over a wide range of scales. The real forcing fields exhibit tremendous variability on large/long and small/short spatiotemporal scales. Sometimes it is possible to identify patterns that can explain some significant fraction of the variability using a set of techniques referred to as empirical orthogonal function analysis or principal 


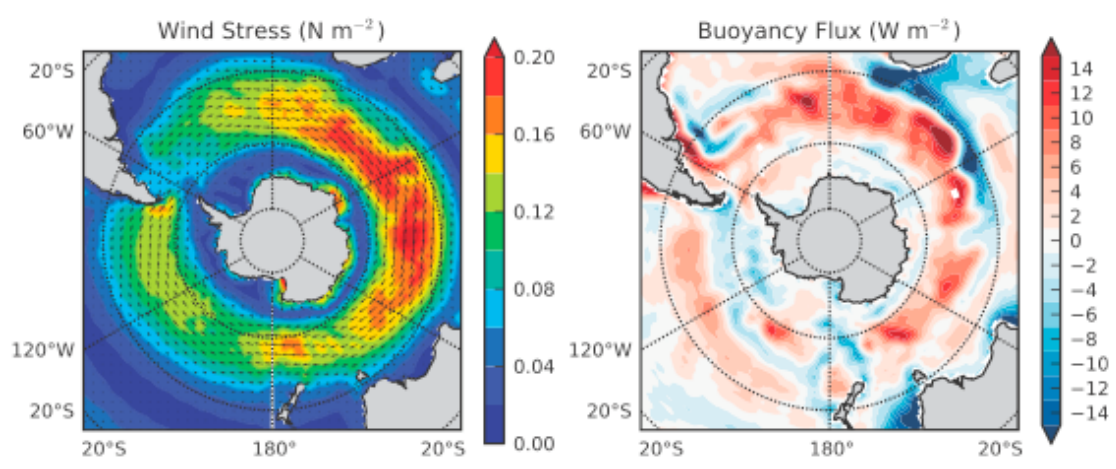

FIG. 1.2. Climatological wind stress (left) and buoyancy (right) forcing taken from the Common Ocean Reference Experiment (CORE2) between 1949-2006 (Large and Yeager, 2009). Figure from Abernathey et al. (2011).

component analysis (EOF/PC) (Lorenz, 1956). When these techniques are applied to surface pressure in the SO, for example, at least two clear patterns (other than the seasonal cycle) emerge. These patterns are called the El Niño - Southern Ocean oscillation (ENSO) and the Southern Annular Mode (SAM) (Figure 1.3). The SAM is so named because it displays considerable zonal symmetry and has a pattern that can be reasonably well described as an annulus. Recent observations suggest the existence of a recent multi-decadal trend in SAM variability that may have important dynamic and climatic consequences (see section 1.3). Before we discuss the trend in the SAM and its possible implications, let us turn our attention to how the surface forcing patterns discussed in this section establish one of the most powerful current systems on Earth.

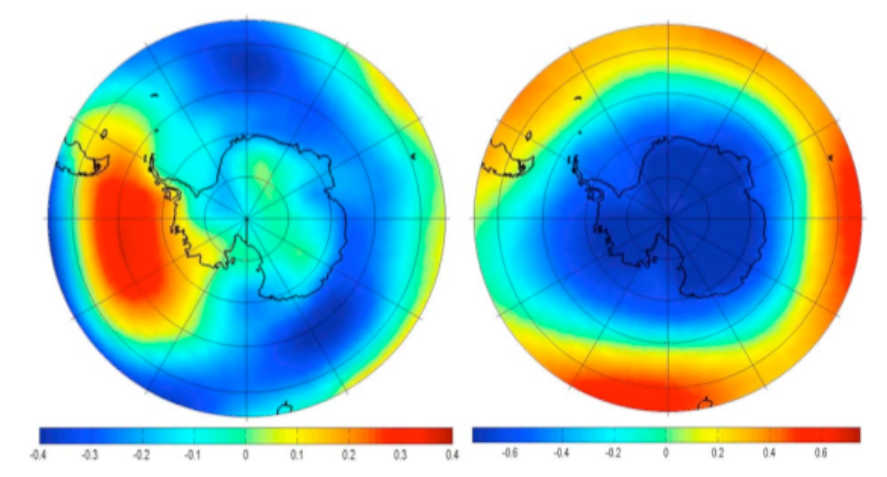

FIG. 1.3. Loading patterns of the El Niño - Southern Ocean oscillation (left) and the Southern Annular Mode (right). Figure from Mayewski et al. (2009). 


\subsection{LARGE-SCALE CIRCULATION AND GLOBAL CLIMATE}

\subsubsection{Currents}

In this section, we discuss the two dominant circulations found in the Southern Ocean, namely the Antarctic Circumpolar Current (ACC) and the meridional overturning circulation (MOC), starting with the ACC. In a time-averaged sense, the ACC can be described as a deep, wide, and relatively slow current that transports a tremendous amount of water around the Antarctic continent. Its average speed at the surface is only about $0.5 \mathrm{~ms}^{-1}$, but since the current extends over $4 \mathrm{~km}$ of depth and can range from 500 - $1000 \mathrm{~km}$ in width, even this slow speed produces a volume transport of around $150 \mathrm{~Sv}\left(1 \mathrm{~Sv}=10^{6} \mathrm{~m}^{3} \mathrm{~s}^{-1}\right)$, which is more than two orders of magnitude larger than the net volume transport of all the world's rivers combined (Fyfe and Saenko, 2005). The ACC is one of Earth's dominant pathways for basin-to-basin propagation of temperature, pressure, velocity, and tracer signals, which on decadal timescales is achieved through the action of long baroclinic Rossby waves and large-scale, low-frequency gravity waves (Cessi and Otheguy, 2003). At first glance, the ACC may appear to be a simple thing to explain. It travels in the same direction as the surface wind stress forcing, after all. However, the manner in which surface forcing establishes the ACC is subtle and still somewhat unclear. For example, the relative importance of wind forcing compared to buoyancy forcing is certainly not immediately obvious and is a current point of considerable debate. Another major impediment to our understanding of this topic is the presence of energetic mesoscale eddies, which resist clean analytical treatments and are computationally expensive to resolve in numerical simulations.

Strong meridional gradients in the SO surface buoyancy flux establish a corresponding north-south density gradient. Thermal wind balance (i.e. the combined result of geostrophy and hydrostatic balance) ensures a nonzero velocity shear and drives the circumpolar current. By this reasoning, it should be possible to establish a circumpolar current without wind stress 
forcing; eddy-permitting numerical simulations with zero wind input can indeed support an ACC-like current (David Munday, personal communication). In the real atmosphere, the wind stress forcing and the buoyancy forcing are coupled; meridional temperature gradients tend to support large-scale wind patterns. In the SO, eastward winds complement the density gradient by creating regions of convergence and divergence in the surface ocean that produce areas of Ekman downwelling and upwelling, respectively. The wind-induced vertical motion further tilts surfaces of constant density (i.e. isopycnals), enhances strong fronts, and supports the ACC. The forcing-driven component of the overturning circulation that tends to tilt isopycnals is referred to as the mean meridional circulation $(\bar{\psi}$ in Figure 1.4$)$.

Tilted isopycnals contain potential energy that can be released through eddy fluxes of isopycnal thickness (a quantity closely related to potential vorticity) via baroclinic instability. The eddy flux of isopycnal thickness is typically thought to be down-gradient, such that thicker-than-average portions of density layers tend to get thinner and thinner-thanaverage portions tend to get thicker. As thickness is redistributed, isopycnal surfaces must by geometric considerations flatten out, and the potential energy once contained in the tilt is now found in energetic mesoscale eddies. The flattening effect can be described by an eddy-induced meridional circulation $\left(\psi^{\prime}\right)$, which is another component of the SO-MOC. In this view, isopycnal tilt in the Southern Ocean (which is related to the stratification across the front) is maintained by a combination of surface forcing and eddy fluxes. The mean circulation $(\bar{\psi})$ tends to steepen the tilt of isopycnals, and the eddy-induced circulation $\left(\psi^{\prime}\right)$ tends to flatten them back out. It is interesting to note that the eddies are not only established by the stratification; they also impact on that same density structure and may alter it in significant ways (Marshall et al. (2001); Karsten et al. (2002)). Some portion of the energy initially found in forcing-driven isopycnal tilt can end up in the mean flow via eddy-mean flow interaction. The net circulation found by adding together the Eulerian mean and eddy-driven streamfunctions is referred to as the residual streamfunction $\left(\psi^{\star}=\bar{\psi}+\psi^{\prime}\right)$. 


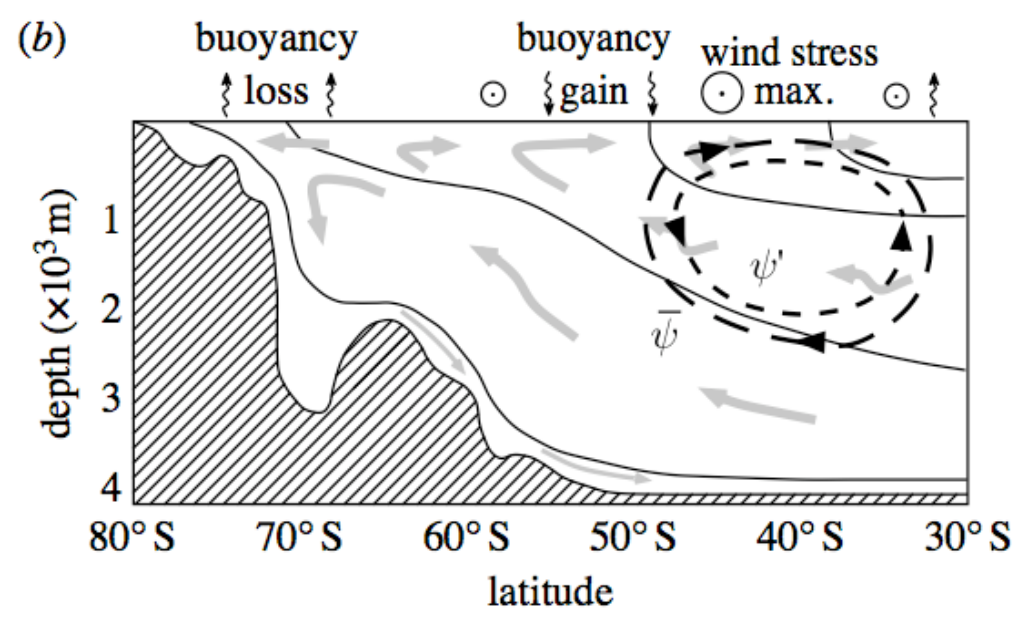

FIG. 1.4. Meridional section of the ACC adapted from Speer et al. (2000). The solid lines are surfaces of constant density and the gray arrows indicate transport. The pattern of surface momentum and buoyancy forcing are shown, as are the Eulerian $(\bar{\psi})$ and eddy-driven $\left(\psi^{\prime}\right)$ streamfunctions. Figure from Thompson (2008).

The residual circulation is what is actually observed in the ocean. It is thought that tracers preferentially spread along surfaces of constant density via the residual circulation (for example, see Figure 5 in Marshall and Speer (2012)).

The meridional overturning streamfunction shown in the upper right portion of Figure 1.4 is embedded within a much larger global overturning circulation, illustrated in Figure 1.5. We can roughly model the global MOC as two counter-circulating cells. In the upper cell, water loses buoyancy to the atmosphere at high northern latitudes (preferentially in the North Atlantic) and sinks, flows mostly along isopycnals in the interior (with some diapycnal mixing), and upwells along the isopycnal outcrop in the Southern Ocean, where it gains buoyancy from the atmosphere and returns northward near the surface. In the lower cell, very cold, dense water is formed near Antarctica, and it must change density classes through diabatic mixing in the interior (Ito and Marshall, 2008). The bottom cell is a clear pathway between the atmosphere and the deep ocean. 


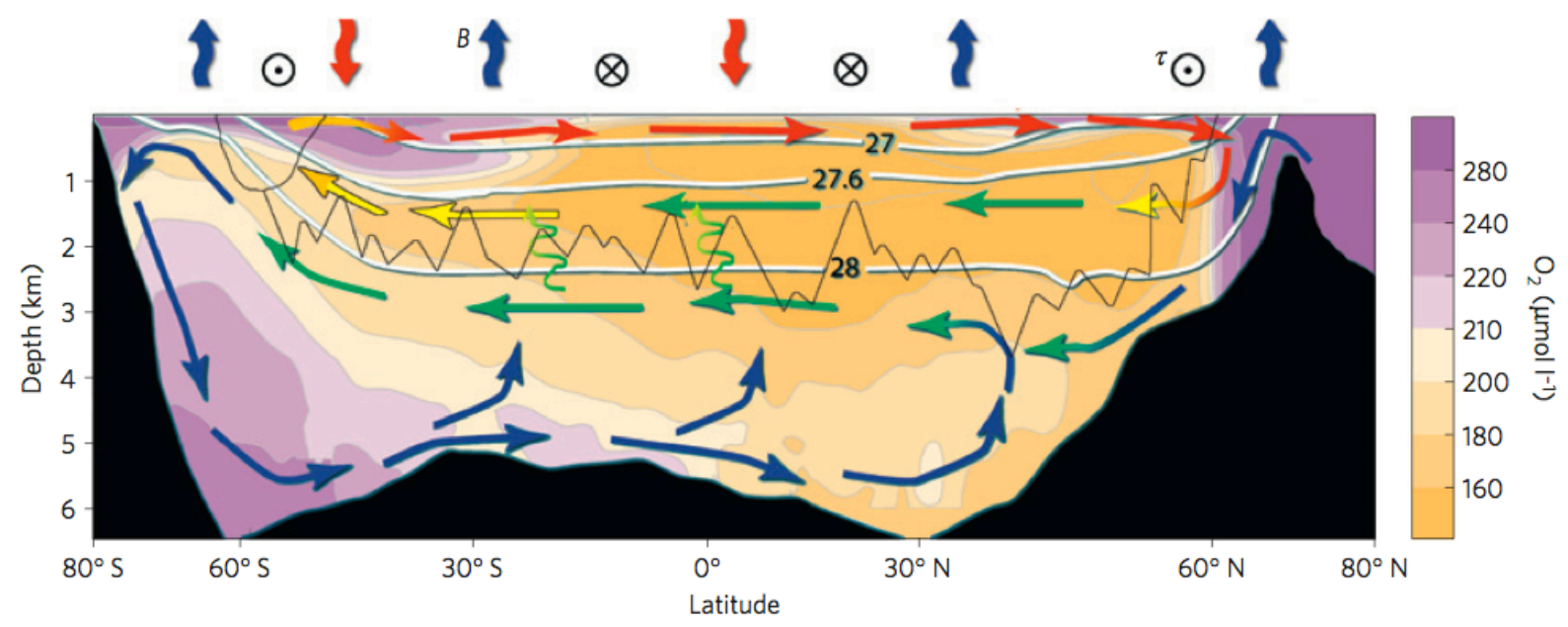

FIG. 1.5. A diagram of the upper and lower cell of the global MOC. The colors of the arrows represent the relative density of the water in the cell, with blue for dense bottom waters, yellow and green for intermediate waters, and red for lighter mode and thermocline waters. The $27.6 \mathrm{kgm}^{-3}$ surface is the approximate divide between the two cells. Squiggly arrows indicate diabatic mixing in the low latitudes. The zonal mean oxygen distribution is shown in the color scale. The jagged black line indicates the depth of the Mid-Atlantic Ridge and the Scotia Ridge. Patterns of surface wind and buoyancy forcing are shown at the top of the plot. Figure from Marshall and Speer (2012).

\subsubsection{The Southern Ocean and global climate}

At present, the oceans absorb more than $25 \%$ of anthropogenic carbon dioxide $\left(\mathrm{CO}_{2}\right)$ emissions, slowing the buildup of atmospheric $\mathrm{CO}_{2}$ (Sabine et al. (2004), LeQuéré et al. (2007)). Strong winds, cold temperatures, biological activity, and a large surface area all help to establish the Southern Ocean as an especially important region of $\mathrm{CO}_{2}$ exchange between the atmosphere and the ocean. Currently, the SO appears to function as a sink of anthropogenic carbon, transporting carbon away from the pole along tilted surfaces of constant density (Toggweiler and Samuels (1995), Sarmiento et al. (2004), Russell et al. (2006)). However, recent observational and modeling studies suggest that the SO carbon sink has weakened in recent decades (Lovenduski et al. (2008), LeQuéré et al. (2007), Metzl (2009)). The weakening is perhaps related to increased upwelling of deep waters rich in 
dissolved organic carbon (DIC), which lowers the difference in the partial pressure of $\mathrm{CO}_{2}$ between the atmosphere and the ocean. Before we can accurately predict the future behavior of the Southern Ocean carbon sink, we must have an idea of how the SO-MOC will evolve in the coming decades and centuries. Both the MOC and the ACC are greatly affected by the presence of mesoscale eddies, which are still not well understood. Though we do not explicitly treat the future evolution of the SO carbon sink in this work, we hope that the results established here may inform future efforts to address this issue.

\subsection{SOUTHERN OCEAN DYNAMICS}

One important goal of physical oceanography is to understand how the large-scale oceanic density field affects and is affected by the turbulent, nonlinear character of fluid flow at the mesoscale. This problem is especially pertinent in the Southern Ocean (SO), where vigorous eddy activity associated with the Antarctic Circumpolar Current (ACC) contains at least as much energy as the mean flow. We are forced to regard both the time-averaged behavior and the departures therefrom (i.e. the mesoscale noise) as being equally relevant to the dynamics and density structure in the SO. The eddy field gives rise to significant variability across a wide range of spatial and temporal scales.

The forced and natural variability of the SO and ACC likely maps onto climate and its variability in a nonlinear fashion (Hogg and Blundell, 2006). On short, monthly timescales there is a connection between the Madden-Julian oscillation in the Indian Ocean and circumpolar transport via wind stress forcing (Matthews and Meredith, 2004). Wind-driven variability is also apparent on sub-annual to annual timescales (Gille et al., 2001). Since the relevant observational records are still somewhat short in length and sparse in coverage, estimating variability on interannual and longer timescales is difficult. For example, the existence of the four-year period Antarctic Circumpolar Wave (White and Peterson, 1996) 
is still a source of considerable debate. However, low-frequency variability is evident in idealized models like that of Wolff et al. (1991), so there is some precedent for the exploration of long-term variability by numerical means.

Several recent studies have attempted to better understand the interannual and multidecadal response of the Southern Ocean to long-term changes in the SAM index. Hogg and Blundell (2006) found a mode of low-frequency variability that depends on the explicit simulation of mesoscale eddy dynamics. Using a global-eddy permitting model run from 1972 to 2001, Treguier et al. (2010) found that the transport of the Antarctic Circumpolar Current (ACC) is correlated with the SAM on interannual time scales but exhibits a drift because of long-term changes in stratification. Allison et al. (2011) employ a 1.5-layer reduced gravity model with parameterized eddies to demonstrate that centennial-scale adjustment takes place through the interaction of the Southern Ocean with the global pycnocline. The adjustment of the Southern Ocean to changing wind stress is clearly a complex, multi-scale problem.

Progress on the long-term adjustment problem has thus far been heavily constrained by computational limitations. Specifically, those studies that use coarse resolution and parameterized eddies cannot accurately represent the impact of mesoscale variability on long-term adjustment, and numerical experiments that use eddy-permitting resolution and a globalscale ocean basin cannot be run for the millennia required for the global ocean to reach thermodynamic equilibrium. The former problem can be addressed using more accurate parameterization schemes, while the latter issue will likely be ameliorated as computational power increases over the next several decades.

In this section, we further outline some of the eddy-driven dynamics at play in the Southern Ocean and how those dynamics may produce variability on interannual and longer timescales. We then examine the eddy compensation and eddy saturation hypotheses and discuss the possibility that these hypotheses may hold on short (interannual) timescales but 
not on longer (decadal to centennial) timescales on which large-scale changes in buoyancy and potential vorticity may be important. We also attempt to clarify the relationship between the residual circulation, eddy diffusivity, and stratification using a simple zonal mean theory.

\subsubsection{Eddies, jets, and fronts}

Observations of the SO reveal the existence of multiple sharp fronts, i.e. places where properties change rapidly in the horizontal. Specifically, geostrophic volume transport fields feature three persistent maxima that correspond to continuous fronts, namely the Soutern ACC Front (SACCF), the Polar Front (PF), and the Sub-Antractic Front (SAF) (Orsi et al., 1995). These fronts can also be located using hydrography data, satellite altimetry data, or SST data (Zhang and Klinck, 2008). Regions of rapid transition are also evident in tracer data, for example in observed temperature, salinity, oxygen, or chlorophyll fields (Moore et al., 1999). The fronts can potentially change position if they are not too strongly constrained by seafloor topography. For example, the meander in the PF is weaker where the bathymetry is steeply sloped and stronger where the bottom is relatively flat (Moore et al., 1999).

Associated with the fronts are steeply tilted surfaces of constant density (i.e. isopycnals). As discussed in the previous section, tilted isopycnals such as those in the Southern Ocean are typically baroclinically unstable, i.e. they generate eddies that tend to adiabatically redistribute isopycnal thickness and flatten density surfaces, acting in opposition to the titling effect of the wind- and buoyancy-driven mean circulation. The eddies derive their energy from the available potential energy associated with isopycnal tilt, which must be continually sustained by a difference in stratification between lower and higher latitudes relative to the position of the front. However, eddies do not simply act as a dissipative mechanism. It is possible for eddies to force the mean flow and encourage the formation of persistent structures, such as strong jets. 
In the theory of geostrophic turbulence, baroclinic instability acts as an effective stirring force, injecting eddy energy into the fluid at the scale of the first baroclinic deformation radius, i.e. the scale on which buoyancy and rotational effects are equally important $\left(L_{d}=\right.$ $N H / f$, where $N$ is the buoyancy frequency, $H$ is a scale height, and $f$ is the Coriolis parameter). The eddies feel the influence of both large-scale straining motions and Rossby waves, both of which may act to redistribute energy between different spatial scales. One important scale for this analysis is the Rhines scale, given by $L_{R}=\sqrt{U / \beta}$, where $U$ is the advective velocity scale and $\beta$ is the planetary curvature (Rhines, 1975). On large spatial scales, the $\beta$-effect is dominant, and on smaller spatial scales advection is dominant. Eddies smaller than the Rhines scale are able to deposit their energy into the large-scale flow through shearing interaction with the mean current, while eddy energy on scales larger than the Rhines scale tends to radiate away as Rossby waves before the mean flow can alter the eddy. The meridional gradient of planetary potential vorticity (i.e. the beta-effect that acts as the restoring force for Rossby waves) tends to halt the cascade of eddy energy towards larger scales and organizes the flow into alternating zonal jets (Salmon, 1998). Since the scale of the first baroclinic deformation radius is smaller in the ocean compared to that of the atmosphere, jets in the SO possess a fine-scale structure and narrow profile relative to the basin size (Hallberg and Gnanadesikan (2006), Sokolov and Rintoul (2009)). These jets exhibit variability on a wide range of timescales, including monthly and interannual scales, and can affect the meridional transport of buoyancy, momentum, and potential vorticity (Thompson and Richards, 2011). The relationship between jet structure and meridional transport of heat and tracers remains an active area of investigation (Ferrari and Nikurashin, 2010). With this more detailed description of eddy activity in mind, we turn our attention back to the sensitivity of the Southern Ocean to changing wind stress. 


\subsubsection{Eddy compensation vs. eddy saturation}

The atmosphere overlying the Southern Ocean has been undergoing significant multidecadal climate change characterized by an intensification and poleward shift of midlatitude westerly winds, which appear to be driven at least in part by ozone depletion and global warming (Thompson and Solomon (2002), Marshall (2003), Miller et al. (2006)). We might also express this trend as a bias towards more positive values of the SAM index indicative of a spin-up of the polar vortex (Thompson and Solomon, 2002). Figure 1.6(a) shows the variation of the eastward surface wind speed averaged over the ocean between $40-50{ }^{\circ} \mathrm{S}$ based on the meteorological reanalysis products of Kalnay et al. (1996), Dee and Uppala (2009), Uppala et al. (2005), and Onogi et al. (2007). Though the magnitude of the wind stress differs among the four observationally-derived products, they all exhibit a positive trend on decadal timescales. The linear component of this trend is superposed with considerable interannual variability.

Since surface Ekman flow is wind-driven, stronger winds should steepen the meridional tilt of density surfaces across the ACC by moving cold, high-latitude water northward. However, recent analysis of float and hydrographic measurements show that the warming of the past several decades (Gille (2002), Gille (2008)) is not correlated with a significant change in the tilt of the isopycnals 1.6(b) (Böning et al., 2008). Böning et al. (2008) hypothesize that an increase in eddy activity may have cancelled out the presumed increased in Ekman flow, greatly reducing the sensitivity of mean isopycnal slope to stronger wind stress (Straub (1993), Völker (1999)). The notion that Southern Ocean meridional overturning circulation and stratification are insensitive to changing wind stress is known as the eddy compensation hypothesis. The closely related (but not identical) eddy saturation hypothesis posits that the volume transport of the ACC is also insensitive to increasing wind stress. Additional energy from the winds is imparted to mesoscale eddies instead of to the acceleration of the mean flow (Hallberg and Gnanadesikan (2006), Meredith and Hogg (2006)). Meredith et al. (2012) 
argue using scaling analysis and numerical simulations that it is possible to satisfy the eddy compensation hypothesis without satisfying the eddy saturation hypothesis, and vice versa.

a.

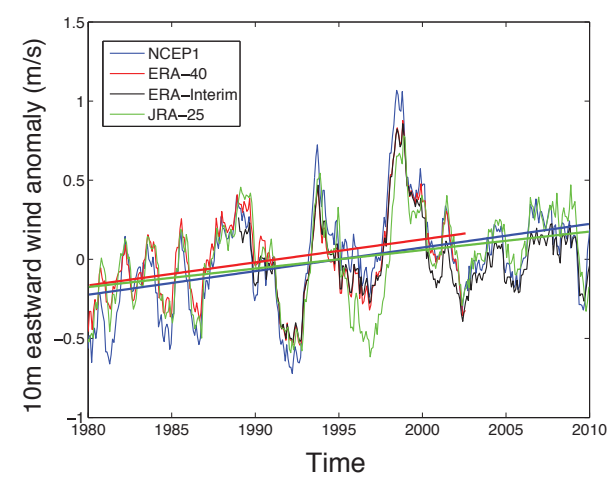

b.

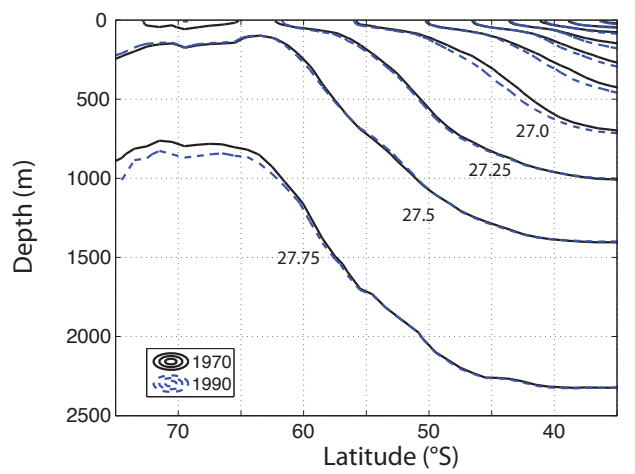

FiG. 1.6. (a) Area-weighted mean eastward wind speed $10 \mathrm{~m}$ above the sea level between 40 ${ }^{\circ} \mathrm{S}$ and $50{ }^{\circ} \mathrm{S}$, based on several meteorological reanalyses. Plotted values are anomalies from the climatological mean values. Thin lines are one-year running mean, and thick lines are linear regressions. (b) Comparison of the zonally averaged ocean density structure between the 1970s and the 1990s based on the ECCO state estimates (Köhl et al., 2007). Contour levels are evenly spaced in potential density $\left(\mathrm{kgm}^{-3}\right)$. Figure from (Jones et al., 2011).

\subsubsection{Testing eddy compensation}

In order for the eddy compensation hypothesis to hold in the strictest, most general sense, any change in the Eulerian mean circulation $(\delta \bar{\psi})$ must be canceled exactly by a corresponding change in the eddy induced circulation $\left(\delta \psi^{\prime}\right)$, such that $\delta \bar{\psi}=-\delta \psi^{\prime}$ and $\delta \psi^{\star}=$ $\delta \bar{\psi}+\delta \psi^{\prime}=0$ on every spatial and temporal scale. That is, the condition $\delta \psi^{\star}=0$ (assuming nonzero mean and eddy induced circulations) requires that the anomalous Eulerian mean and eddy induced circulations be identical in spatial structure and response timescale. This is a difficult condition to satisfy in general, since the two circulations have differing vertical structures (Meredith et al., 2012). 
The magnitude of the meridional overturning circulation is expected to be linear with wind stress on short (intra-annual) timescales, as observed in large-scale ocean models (Fyfe and Saenko (2005), Ito et al. (2010)). The degree of compensation achieved by the eddy induced circulation is not clear, especially on decadal and longer scales that are expensive to compute numerically. Hallberg and Gnanadesikan (2006) showed that eddies can indeed compensate for a great deal of Ekman transport variability, though it should be kept in mind that wind-driven transport is only a part of the entire meridional overturning circulation. Farneti et al. (2010) also found that eddies can act as a buffer to changes in surface wind stress, such that the ACC and MOC are less sensitive than they would be without eddies. Treguier et al. (2010) demonstrated that the zonal mean and eddying circulations are governed by different mechanisms on interannual versus decadal timescales, which supports the possibility that eddy compensation and/or saturation hold over some spatiotemporal averaging scales but not over others.

Drawing on the work of Andrews and McIntyre (1976), Marshall and Radko (2003) constructed a zonal mean theory for the residual streamfunction using along-streamline zonal averaging. In this theory, the wind-driven (Eulerian mean) circulation is given by $\bar{\psi}=$ $-\tau / f$, where $\tau$ is the surface wind stress and $f$ is the planetary vorticity. The eddy-induced circulation is given by $\psi^{\prime}=\overline{v^{\prime} b^{\prime}} / \overline{b_{z}}$, where the numerator represents the meridional eddy flux of buoyancy and $\overline{b_{z}}=\partial \bar{b} / \partial z$ is the vertical stratification. Using the simple down-gradient closure scheme of Gent and Mcwilliams (1990), the interior eddy buoyancy flux can be written $\overline{v^{\prime} b^{\prime}}=-K \overline{b_{y}}$, where $K$ is the eddy transfer coefficient that includes contributions from both eddy transport and eddy diffusivity. The eddy-induced circulation becomes

$$
\psi^{\prime}=\frac{\overline{v^{\prime} b^{\prime}}}{\overline{b_{z}}}=-K \frac{\overline{b_{y}}}{\overline{b_{z}}}=K s_{\rho},
$$

where $s_{\rho}$ is the slope of mean buoyancy surfaces. Following Visbeck et al. (1997), we assume that the eddy transfer coefficient is itself a function of isopycnal slope, i.e. $K=k\left|s_{\rho}\right|$, where $k$ is a positive scaling constant. Using these assumptions we can write the residual circulation 
as

$$
\psi^{\star}=\bar{\psi}+\psi^{\star}=-\frac{\tau}{f}+k\left|s_{\rho}\right| s_{\rho}
$$

In the Southern Ocean, the isopycnal slope is largely negative, so we restrict our attention to $s_{\rho}<0$ such that $\left|s_{\rho}\right|=-s_{\rho}$. The residual circulation becomes

$$
\psi^{\star}=\bar{\psi}+\psi^{\star}=-\frac{\tau}{f}-k s_{\rho}^{2}
$$

The steady-state slope is then

$$
s_{\rho}=-\left[-\frac{\tau}{f k}-\frac{\psi^{\star}}{k}\right]^{1 / 2},
$$

and the sensitivity of the residual circulation to a change in wind stress is

$$
\frac{\partial \psi^{\star}}{\partial \tau}=-\frac{1}{f}-2 k s_{\rho} \frac{\partial s_{\rho}}{\partial \tau}
$$

Assuming that the chosen eddy parameterization scheme is correct, equation 1.1 suggests that it is impossible for both the residual circulation and the isopycnal slope to be insensitive to wind stress, since in the limit that $\partial_{\tau} \psi^{\star}=0$ and $\partial_{\tau} s_{\rho}=0, f$ cannot be finite. The sensitivity of the slope to wind stress and the sensitivity of the residual circulation to wind stress are different (albeit related) quantities. Observations suggest that $\partial_{\tau} s_{\rho}=0$ on interannual timescales, such that the sensitivity of the residual circulation to wind stress is constant and positive in the Southern Hemisphere. How is it physically possible for the residual circulation to strengthen without a corresponding change in isopycnal slope? One solution is for the isopycnals to shift vertically without tilting, such that the slope remains constant.

It may be the case that our eddy parameterization scheme is not appropriate for longterm adjustment. In general, the eddy diffusivity $K=-\overline{v^{\prime} b^{\prime}} / \overline{b_{y}}$ may be able to strengthen through the eddy flux $\overline{v^{\prime} b^{\prime}}$ without a corresponding change in stratification $\overline{b_{y}}$. This may happen if a significant fraction of the extra power input from increased winds ends up in the eddy kinetic energy as opposed to the energy of the mean flow. If we keep $K$ general and 
do not assume a particular parameterization scheme, the wind-stress sensitivity equation 1.1 becomes

$$
\frac{\partial \psi^{\star}}{\partial \tau}=-\frac{1}{f}+s_{\rho} \frac{\partial K}{\partial \tau}+K \frac{\partial s_{\rho}}{\partial \tau} .
$$

The mean slope field may take many years to decades to respond to a change in wind stress, so on inter-annual timescales the last term is small and will not be able to balance $1 / f$. In the limit of perfect eddy compensation $\left(\partial_{\tau} \psi^{\star}=0\right.$ and $\left.\partial_{\tau} s_{\rho}=0\right)$, eddy diffusivity must change with wind stress in this simple theory:

$$
0=-\frac{1}{f}+s_{\rho} \frac{\partial K}{\partial \tau}
$$

If equation 1.3 represents the dominant balance, then the sensitivity of the slope to wind stress must balance with the sensitivity of the residual circulation in equation 1.2. Note that this theory uses along streamline averaging and therefore does not contain contributions from any standing wave pattern or other stationary feature. It is also a local theory, in that it does not explicitly describe the connection between Southern Ocean isopycnal slope $s_{\rho}$ and global stratification. Despite these limitations, this theory clarifies the relationship between the residual circulation, diffusivity, and isopycnal slope in the Southern Ocean.

\subsection{DISSERTATION OBJECTIVES AND STRUCTURE}

In this work, we test the validity of the eddy compensation and saturation hypotheses using a combination of simple conceptual models and a numerical ocean general circulation model. In Chapter 2, we develop a simple model of the manner in which the Southern Ocean adjusts with the world ocean through the global pycnocline, which may be the rate-limiting step in the equilibration of the SO to changes in wind stress. We test the predictions of the simple model using an oceanic general circulation model (OGCM) with idealized bathymetry and surface forcing (described in Chapter 3), and we find that both eddy-parameterized (Chapter 4) and eddy-permitting (Chapter 5) variants exhibit multi-

decadal buoyancy structure adjustment, though the presence of explicitly resolved eddies 
somewhat enhances compensation. In an effort to better understand interannual variability of eddying flow to a change in surface wind forcing, we perform several numerical experiments with sinusoidal forcing (discussed in Chapter 6) and examine correlations between forcing and northward heat transport, among other diagnostic quantities. The results of this study and directions for future work are summarized in Chapter 7. Our results suggest that eddy compensation and eddy saturation are not especially strong constraints on ocean circulation, and departures from the compensation/saturation regime may be important on multi-decadal timescales and longer. We suspect that the overturning circulation will increase in response to decadal wind stress variability, with implications for the Southern Ocean carbon sink and global climate. 


\section{HOW DOES THE SOUTHERN OCEAN INTERACT WITH THE GLOBAL OCEAN?}

The main pycnocline is the layer within which density changes rapidly with depth. It is the boundary between the warm, light surface waters that sit at low latitudes and the cold, dense waters that form at high latitudes and fill the abyss. Though its position can change with time, it is considered a permanent feature of oceanic density structure. We might say that it is quasi-stationary. The processes that determine the structure of the pycnocline and its long-term variability are under debate here.

In this chapter, we consider a simple, time-dependent, two-box model of the oceanic density structure that predicts both the sensitivity and the characteristic response timescale of pycnocline depth to various physical parameters. We include a generalized dependence of eddy-driven Southern Ocean transport on pycnocline depth, since this sensitivity is not yet well constrained. Our focus is on the sensitivity of pycnocline depth to Southern Ocean wind stress. We find that the global pycnocline typically deepens with increasing wind stress, reflecting an enhanced conversion of dense water to light water (i.e. diabatic circulation) in the Southern Ocean following an increase in equatorward Ekman transport. The characteristic response timescale of pycnocline depth to changing winds is on the order of decades to centuries, depending on the parameters used. Furthermore, our model predicts that increasing the sensitivity of Southern Ocean eddy transport to wind stress (i.) weakens the deepening of the pycnocline and (ii.) shortens the characteristic response timescale.

\subsection{OBSERVATIONS OF THE GLOBAL PYCNOCLINE}

The climatological mean picture of the ocean features fluid in a stably stratified configuration. The uneven density field shown in 2.1 suggests that we can crudely divide the ocean into two layers. In the first 500-1000 m, we find a layer where temperature drops 
rapidly with depth. This layer is called the pycnocline, and its thickness is an important parameter of the climate system. Resting on top of this bowl of relatively warm, light water sits cold, dense abyssal water that forms at high latitudes and fills the deep ocean. The vertical temperature gradient in the deep ocean is much smaller than that in the pycnocline. We want to determine what processes set the structure of the pycnocline and how those

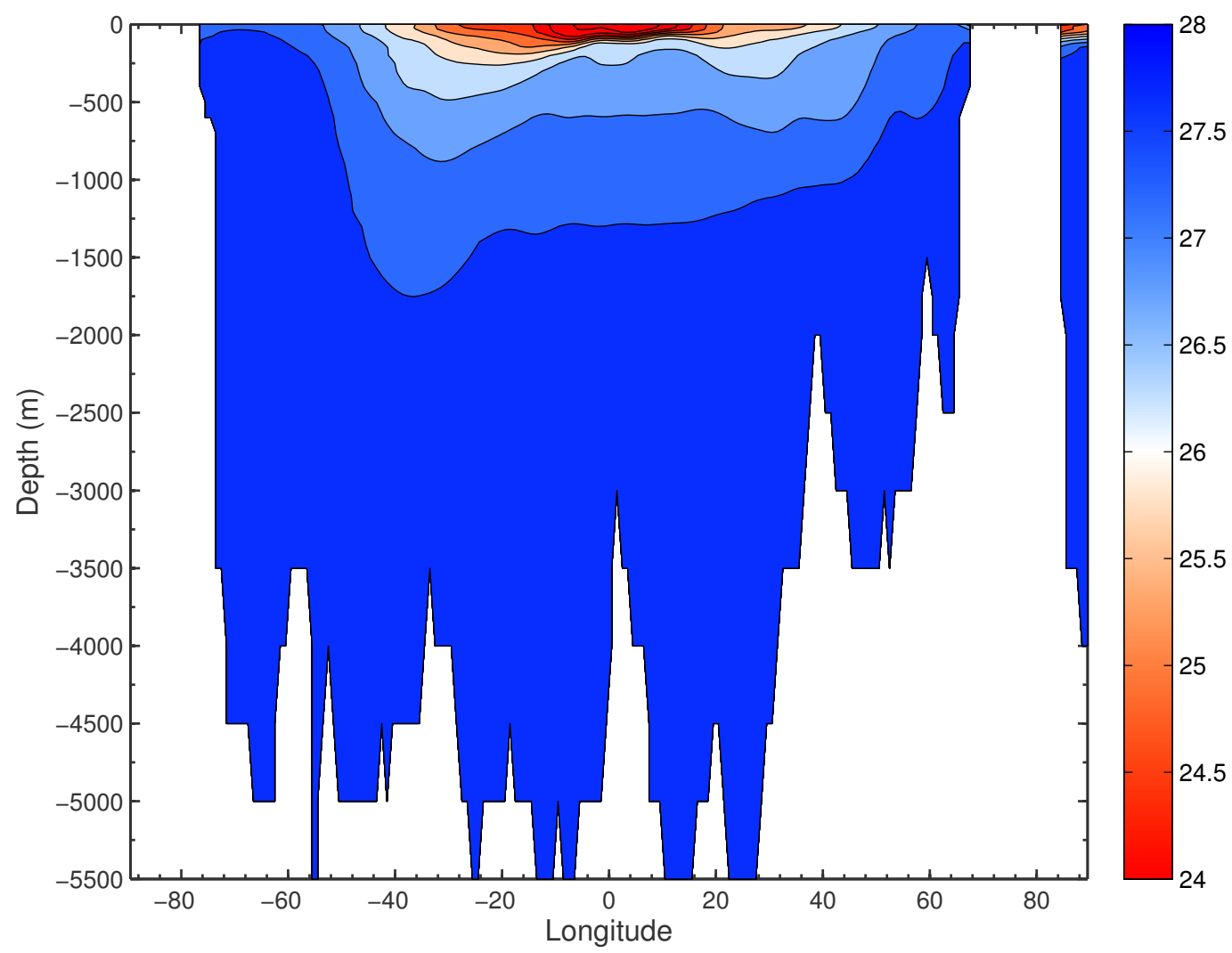

FIG. 2.1. $\sigma=\rho-\rho_{0}, \rho_{0}=1000 \mathrm{~kg} / \mathrm{m}^{3}$ in the water column, cut through the Atlantic. Data from Levitus World Ocean Atlas 1998.

processes depend on surface forcing. In the next section, we discuss a box model built to deal with this problem. 


\subsection{A SIMPLE MODEL OF PYCNOCLINE STRUCTURE}

Here we develop a highly idealized two-box model of oceanic density structure based on the work of Gnanadesikan (1999). The geometry and relevant fluxes are shown in Figure 2.2. We assume that the included fluxes are the only ones relevant for establishing and maintaining the large-scale structure of the pycnocline. We include the formation of deep water at high northern latitudes $\left(T_{N}\right)$, upwelling of deep water into the pycnocline in the

tropics and subtropics $\left(T_{U}\right)$, wind-driven Ekman transport $\left(T_{E k m a n}\right)$, and poleward eddy fluxes in the Southern Ocean $\left(T_{E d d y}\right)$. The model may be thought of as somewhat of a zonal mean picture, such that contributions from the upwelling flux may come from any tropical ocean basin, the Southern Ocean fluxes represent a global zonal mean, and northern sinking occurs primarily in the Atlantic (deep water forms more readily there because the Atlantic is saltier than the Pacific). The global pycnocline depth $D$ is set by these fluxes and is characterized by a single value in this model. In reality, the pycnocline has complex spatial structure in that it may change with latitude and longitude. We ignore that complication for now in order to get a simpler first-order model. The formation and upwelling of Antarctic Bottom Water (AABW) is also not included in this model, since the circulation associated with AABW sits below the main pycnocline. That is, we assume that the upper meridional circulation cell does not depend strongly on the strength of the lower cell. Let us now discuss the individual volume fluxes in greater detail.

\subsubsection{Low-latitude upwelling}

The buoyancy equation is

$$
\frac{\partial b}{\partial t}+\nabla \cdot(b \mathbf{v})=J+K_{v} \nabla^{2} b,
$$

where $J$ is the rate of heating/cooling and $K_{v}$ is the vertical eddy diffusivity. We assume that there is no flux through horizontal boundaries. Integrating horizontally in the interior 


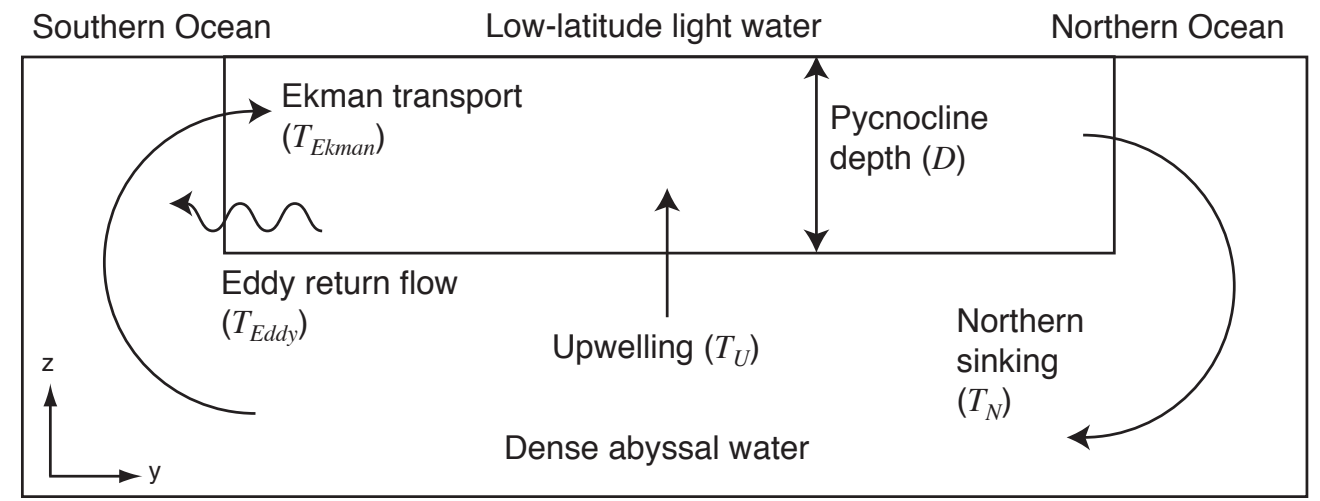

Fig. 2.2. Diagram of the two-box model with fluxes $T$ and pycnocline depth $D$. Adapted from Gnanadesikan (1999).

produces

$$
\frac{\partial \bar{b}}{\partial t}+\bar{w} \frac{\partial \bar{b}}{\partial z}=K_{v} \frac{\partial^{2} \bar{b}}{\partial z^{2}}
$$

Under steady state conditions $\left(\partial_{t} b=0\right)$, a balance exists between the downward diffusion of buoyancy and upward advection of dense water. If we adopt $W$ as a typical vertical velocity scale and $D$ as the depth scale of the pycnocline, we get $W=K_{v} / D$. Scaling by the upwelling area gives us the volume transport due to low-latitude upwelling,

$$
T_{u}=W A=\frac{K_{v} A}{D}
$$

The flux $T_{u}$ represents the conversion from dense abyssal water to light surface water that occurs in the tropics and subtropics.

\subsubsection{North Atlantic deep water formation}

Differential heating produces a sea surface height difference between warmer, low latitude water and colder water in the North Atlantic. This gives rise to a horizontal pressure gradient of the form

$$
\frac{\Delta p}{\rho} \propto \frac{\Delta \rho g D}{\rho}=g^{\prime} D
$$


where $g^{\prime}$ is defined as the reduced gravity. The reduced gravity factor represents the effects of buoyancy forcing at the surface. The pressure gradient force associated with northern sinking is balanced by friction at the western boundary current (WBC),

$$
A_{h} \frac{\partial^{2} v}{\partial x^{2}}=\frac{1}{\rho} \frac{\partial p}{\partial y} \rightarrow A_{h} \frac{V}{L_{m}^{2}}=\frac{\Delta p}{\rho L_{y}^{N}}
$$

where $A_{h}$ is the coefficient of eddy viscosity, $L_{m}$ is the width of the WBC, $V$ is a characteristic velocity scale for the WBC, and $L_{y}^{N}$ is the north-south distance over which a gradient in layer depth occurs. Using our pressure gradient expression and the scale for the width of the boundary current $L_{m}=\left(A_{h} / \beta\right)^{1 / 3}$ from classical boundary layer theory, we get

$$
A_{h}^{1 / 3} \beta^{2 / 3} V=C \frac{g^{\prime} D}{L_{y}^{N}}
$$

where $C$ is a constant that incorporates the effects of geometry and boundary layer structure. We can then write the volume flux associated with the formation of the North Atlantic Deep Water as

$$
T_{N}=V D L_{m}=\frac{C g^{\prime} D^{2}}{\beta L_{y}^{N}}
$$

\subsubsection{Southern Ocean dynamics}

The net volume flux associated with Southern Ocean processes is given by the balance between the equatorward wind-driven Ekman transport and the poleward eddy-driven mass flux. Ekman transport tends to steepen subsurface isopycnals diabatically while the eddy flux, brought about by the release of available potential energy via eddies generated through instability, tends to flatten them back out. The net Southern Ocean flux can be written

$$
T_{S}=T_{E k m a n}-T_{e d d y}=\left(\frac{\tau}{\rho|f|}-\frac{A_{I} D}{L_{y}^{S}}\right) L_{x}
$$

where $\tau$ is the southern ocean wind stress, $f$ is the Coriolis parameter, $A_{I}$ is an eddy diffusion coefficient, and $L_{x}$ is the circumference of the Earth in Drake passage latitudes. 
In equation 2.5, we have parameterized the eddy bolus velocity as being proportional to the slope of surfaces of constant density (and therefore proportional to $D$ if $L_{y}^{S}$ remains approximately constant),

$$
v_{\text {eddy }}=A_{I} \times(\text { slope }) \propto A_{I} \frac{D}{L_{y}^{S}} .
$$

Alternatively, we could allow the eddy-induced transport velocity $v_{e d d y}$ to be more sensitive to the pycnocline depth (and therefore more sensitive to the isopycnal slope). That is, we could generalize the eddy bolus velocity as

$$
v_{e d d y} \propto A_{I} \frac{D^{n}}{L_{y}^{S} D_{r e f}^{n-1}},
$$

where $D_{\text {ref }}$ is a reference equilibrium depth. This generalized parameterization is reasonable at this stage of our investigation, because the sensitivity of eddy transport to isopycnal slope is complex and still somewhat poorly constrained. Roughly speaking, the case $n=1$ corresponds to the parameterization scheme of Gent and Mcwilliams (1990) and the case $n=2$ to that of Visbeck et al. (1997). Using the generalized sensitivity in (2.5), we can write the volume flux as

$$
T_{S}=T_{E k m a n}-T_{e d d y}=\left(\frac{\tau}{\rho|f|}-\frac{A_{I} D^{n}}{L_{y}^{S} D_{r e f}^{n-1}}\right) L_{x} .
$$

Now that we have developed scaling relationships for the four major volume fluxes under consideration, we can consider a steady-state condition in which the net volume flux into the layer of light, low-latitude surface water is equal to the net flux out of it.

\subsection{EQUILIBRIUM PYCNOCLINE DEPTH}

In equilibrium $\left(d_{t} D=0\right)$, the flux of water entering the low-latitude bowl of light water must equal the flux of water leaving this bowl. That is, we must have

$$
T_{U}+T_{S}-T_{N}=0
$$


Using expressions 2.3, 2.4, and 2.6, we get

$$
\frac{K_{v} A}{D_{0}}+\frac{\tau L_{x}}{\rho f}-\frac{A_{I} L_{x} D_{0}^{n}}{L_{y}^{S} D_{r e f}^{n-1}}-\frac{C g^{\prime} D_{0}^{2}}{\beta L_{y}^{N}}=0
$$

where $D_{0}$ is the equilibrium pycnocline depth. Multiplying through by $-D_{0}$ and dividing by $A_{I} L_{x} / L_{y}^{S} D_{r e f}^{n-1}$, we get

$$
D_{0}^{n+1}+\left(\frac{L_{y}^{S} D_{r e f}^{n-1} C g \prime}{L_{y}^{N} L_{x} A_{I} \beta}\right) D_{0}^{3}-\left(\frac{L_{y}^{S} D_{r e f}^{n-1} \tau}{A_{I} \rho|f|}\right) D_{0}-\frac{L_{y}^{S} D_{r e f}^{n-1} A}{A_{I} L_{x}} K_{v}=0
$$

which is a polynomial of degree $\max (3, n+1)$ of the form $D_{0}^{n+1}+a D_{0}^{3}-b D_{0}-c=0$. Note that $a, b$, and $c$ are positive constants that depend on $n$. In equation 2.7 there is only one sign change as you proceed from the highest power to the lowest, so by Descartes' sign rule, equation 2.7 has at most one positive root. This is reasonable, since only positive roots are physically meaningful. However, this result excludes the possibility of multiple equilibria. There is no analytical form for the general solution of 2.7. However, we can write down the solution for a few special cases, discussed in the following subsections.

\subsubsection{Linear transport sensitivity}

For the special case $n=1$, equation 2.7 becomes

$$
a D_{0}^{3}+D_{0}^{2}-b D_{0}-c=0
$$

or, writing it in basic form,

$$
D_{0}^{3}+a_{2} D_{0}^{2}-a_{1} D_{0}-a_{0}=0
$$

where $a_{2}=1 / a, a_{1}=b / a$, and $a_{0}=c / a$. The solution to 2.8 can be written down analytically, though the form is a bit messy. The substitution $y=x+a_{2} / 3$ leads to the reduced form

$$
y^{3}+p y+q=0
$$


where $p=\left(-3 a_{1}-a_{2}^{2}\right) / 3$ and $q=-a_{0}+2 a_{2}^{3} / 27+a_{2} a_{1} / 3$. If we define the polynomial discriminant to be

$$
\Delta=\left(\frac{p}{3}\right)^{3}+\left(\frac{q}{2}\right)^{2}
$$

then we can write the solution to 2.8 using Cardano's formulas,

$$
\begin{aligned}
x_{1} & =-\frac{a_{2}}{3}+u+v \\
x_{2,3} & =-\frac{a_{2}}{3}-\frac{u+v}{2} \pm \sqrt{3} \frac{u-v}{2} i
\end{aligned}
$$

where

$$
u=\left(-\frac{q}{2}+\sqrt{\Delta}\right)^{1 / 3}
$$

and

$$
v=\left(-\frac{q}{2}-\sqrt{\Delta}\right)^{1 / 3}
$$

If $\Delta<0$, then 2.8 has three distinct real roots that can be written in a simplified trigonometric form (Harris and Stocker, 1998).

\subsubsection{Quadratic transport sensitivity}

For $n=2$, equation 2.7 becomes $(1+a) D_{0}^{3}-b D_{0}-c=0$, or, in standard form,

$$
D_{0}^{3}-a_{1} D_{0}-a_{0}=0
$$

where $a_{1}=b /(1+a)$ and $a_{0}=c /(1+a)$. This is already a depressed cubic, so the solution is the same as in the $n=1$ case, except with $p=-a_{1}$ and $q=-a_{0}$. The discriminant takes the simple form

$$
\Delta=-\left(\frac{a_{1}}{3}\right)^{3}+\left(\frac{a_{0}}{2}\right)^{2}
$$

and the roots of 2.11 are given by 2.9 and 2.10 . We can also write down an analytical solution for the $n=3$ case, since this produces a quartic equation in $n$. However, for $n>3$, no analytic solution exists, so we must resort to numerical methods to investigate the roots of 2.7 . 


\subsubsection{Higher-order transport sensitivity}

In Figure 2.3, we plot the roots of 2.7 for varying values of the wind stress $\tau$ and transport sensitivity coefficient $n$. The physical parameters are set to values appropriate for the global ocean and are given in Table 2.1. Increasing wind stress generally increases the equilibrium depth $D_{0}$ of the pycnocline, but $D_{0}$ becomes less sensitive to increasing wind stress with increasing $n$. This seems reasonable, as larger values of $n$ correspond to regimes of eddy transport that are more sensitive to the tilt of the isopycnals and are therefore better able to compensate for any wind-induced changes in isopycnal tilt. Larger $n$ corresponds to stronger eddy compensation.

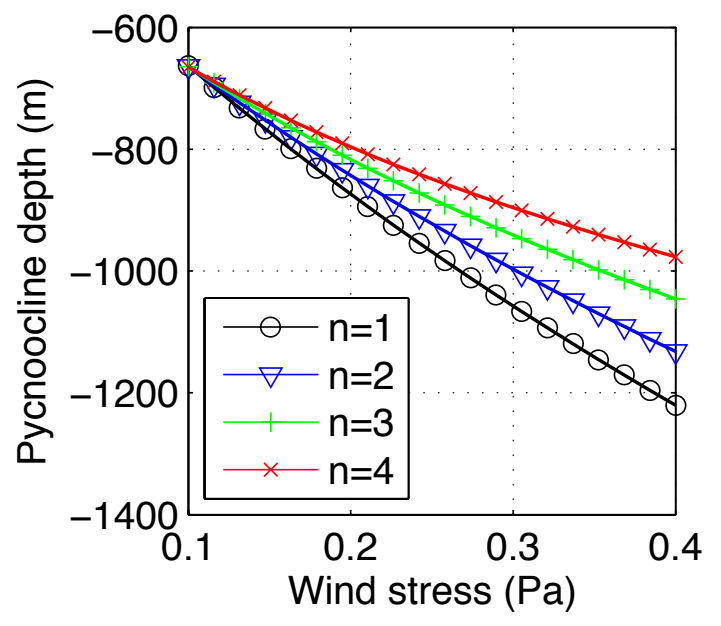

FIG. 2.3. Equilibrium pycnocline depth as a function of wind stress and nonlinearity parameter predicted by the simple model.

\subsection{ADJUSTMENT OF THE GLOBAL PYCNOCLINE}

So far, we have only examined the steady state behavior of the main oceanic pycnocline. In this section, we analyze the behavior of the pycnocline depth when it is out of equilibrium. 
To do this, we extend our model to include time dependence. We assume that the time evolution of the pycnocline is set by the residual of the volume fluxes between the light and deep box,

$$
A \frac{d D}{d t}=-T_{N}+T_{U}+T_{S}
$$

where $A$ is the low-latitude upwelling area. Using the scaling values developed in previous sections, we can write

$$
\frac{d D}{d t}=-\frac{B_{N}}{A} D^{2}+\frac{B_{U}}{A D}+\frac{B_{W}}{A}-\frac{B_{E}}{A} D^{n}
$$

where we have grouped the physical constants into the coefficients $B$. After absorbing the upwelling area $A$ into the constants $B$, we can write

$$
\dot{D}(t)=-C_{E} D^{n}(t)-C_{N} D^{2}(t)+C_{W}+\frac{C_{U}}{D(t)} .
$$

Equation 2.12 constitutes our time-dependent model of the global pycnocline depth.

\subsubsection{Closed-form solution}

Note that equation 2.12 is a separable, first-order, nonlinear ordinary differential equation. A closed form solution is possible, though it will probably be implicit in form (Yan

Table 2.1. Parameters used in the global configuration

\begin{tabular}{ll}
\hline Parameter \\
\hline$L_{x}$ & $25000 \mathrm{~km}$ \\
$L_{y}^{N}, L_{y}^{S}$ & $1500 \mathrm{~km}$ \\
$A$ & $2.44 \times 10^{14} \mathrm{~m}^{2}$ \\
$f$ & $1.0 \times 10^{-4} 1 / \mathrm{s}$ \\
$\beta$ & $2 \times 10^{-11} 1 / \mathrm{ms}$ \\
$K_{v}$ & $3 \times 10^{-5} \mathrm{~m}^{2} / \mathrm{s}$ \\
$A_{I}$ & $10^{3} \mathrm{~m}^{2} / \mathrm{s}$ \\
$\rho$ & $1035 \mathrm{~kg} / \mathrm{m}^{3}$ \\
$C$ & 0.16 \\
$g^{\prime}$ & $0.01 \mathrm{~m} / \mathrm{s}^{2}$ \\
$D_{r e f}$ & $670 \mathrm{~m}$ \\
\hline
\end{tabular}


Wu, personal communication). Separating the ODE and integrating both sides, we get

$$
\int \frac{D d D}{-C_{E} D^{n+1}-C_{N} D^{3}+C_{W} D+C_{U}}=\int d t
$$

Note that the poles of the rational function in the LHS are the equilibrium states of 2.12, which are the roots of 2.7. Once we have these equilibrium states, the LHS can be evaluated by partial fraction decomposition. Let's take a look at a couple of special cases.

\subsubsection{Linear and quadratic dependence}

Assume that the three roots of 2.8 are distinct. If we write those roots as $D_{0}^{(1)}, D_{0}^{(2)}$, and $D_{0}^{(3)}$, then we can write 2.12 as

$$
\int \frac{D d D}{\left(D-D_{0}^{(1)}\right)\left(D-D_{0}^{(2)}\right)\left(D-D_{0}^{(3)}\right)}=\int d t .
$$

Next, we can rewrite the rational function on the LHS using partial fractions,

$$
R(D)=\frac{D}{\left(D-D_{0}^{(1)}\right)\left(D-D_{0}^{(2)}\right)\left(D-D_{0}^{(3)}\right)}=\frac{A_{0}^{(1)}}{D-D_{0}^{(1)}}+\frac{A_{0}^{(2)}}{D-D_{0}^{(2)}}+\frac{A_{0}^{(3)}}{D-D_{0}^{(3)}},
$$

where

$$
A_{0}^{(k)}=\lim _{D \rightarrow D_{0}^{(k)}}\left(D-D_{0}^{(k)}\right) R(D)
$$

After integrating each term, we get the implicit solution

$$
A_{0}^{(1)} \ln \left(D-D_{0}^{(1)}\right)+A_{0}^{(2)} \ln \left(D-D_{0}^{(2)}\right)+A_{0}^{(3)} \ln \left(D-D_{0}^{(3)}\right)=t+E,
$$

where $E$ is a constant of integration and

$$
\begin{aligned}
& A_{0}^{(1)}=\frac{D_{0}^{(1)}}{\left(D_{0}^{(1)}-D_{0}^{(2)}\right)\left(D_{0}^{(1)}-D_{0}^{(3)}\right)}, \\
& A_{0}^{(2)}=\frac{D_{0}^{(2)}}{\left(D_{0}^{(2)}-D_{0}^{(1)}\right)\left(D_{0}^{(2)}-D_{0}^{(3)}\right)}, \\
& A_{0}^{(3)}=\frac{D_{0}^{(3)}}{\left(D_{0}^{(3)}-D_{0}^{(1)}\right)\left(D_{0}^{(3)}-D_{0}^{(2)}\right)} .
\end{aligned}
$$

Equation 2.14 is also the form of the solution when $n=2$, since there are still three steady states. Only the values taken on by the equilibrium points will change. Note that the equilibrium points $D_{0}^{(k)}$ are, in general, complex. 


\subsubsection{Higher-order sensitivity}

For $n \geq 3$, equation 2.7 becomes an equation of order $n+1$, with $n+1$ roots. Thus, our rational function $R(D)$ is

$$
R(D)=\frac{D}{\left(D-D_{0}^{(1)}\right)\left(D-D_{0}^{(2)}\right) \cdots\left(D-D_{0}^{(n+1)}\right)}=\sum_{k=1}^{n+1} \frac{A_{0}^{(k)}}{D-D_{0}^{(k)}},
$$

such that (2.13) becomes

$$
\sum_{k=1}^{n+1} \int \frac{A_{0}^{(k)}}{D-D_{0}^{(k)}} d D=\sum_{k=1}^{n+1} A_{0}^{(k)} \ln \left|D-D_{0}^{(k)}\right|=t+E .
$$

Recall that we have assumed that the roots of 2.7 are distinct. This is a reasonable assumption, since we only have repeated roots when the discriminant $\Delta=0$, which only happens in a very small slice of parameter space. As $t$ becomes very large, the pycnocline depth $D$

must approach one of the equilibria $D_{0}^{(k)}$ such that the argument of the logarithm becomes small and the log itself becomes very large. A small pycnocline depth anomaly exponentially decays away with time.

In Figure 2.4, we plot the evolution of the pycnocline depth predicted by equation 2.12 for four different values of $n$ using an initial depth far from the equilibrium value. In all four cases, the pycnocline depth deepens to its equilibrium value over the course of several centuries. Higher values of $n$ produce thinner pycnoclines with shorter adjustment timescales and lower values of $n$ produce thicker pycnoclines with longer adjustment timescales. In Figure 2.5, we plot the behavior of individual terms in equation 2.12 for the first four centuries of adjustment. The upwelling $T_{u}$ and eddy flux $T_{E d d y}$ terms appear to be fully adjusted after about a century. The northern sinking term $T_{n}$ dominates the century-scale adjustment since $T_{n}$ is the slowest of the four terms to fully equilibrate. Ekman transport $T_{E k m a n}$ does not depend on the pycnocline depth, so it does not depend on time. 


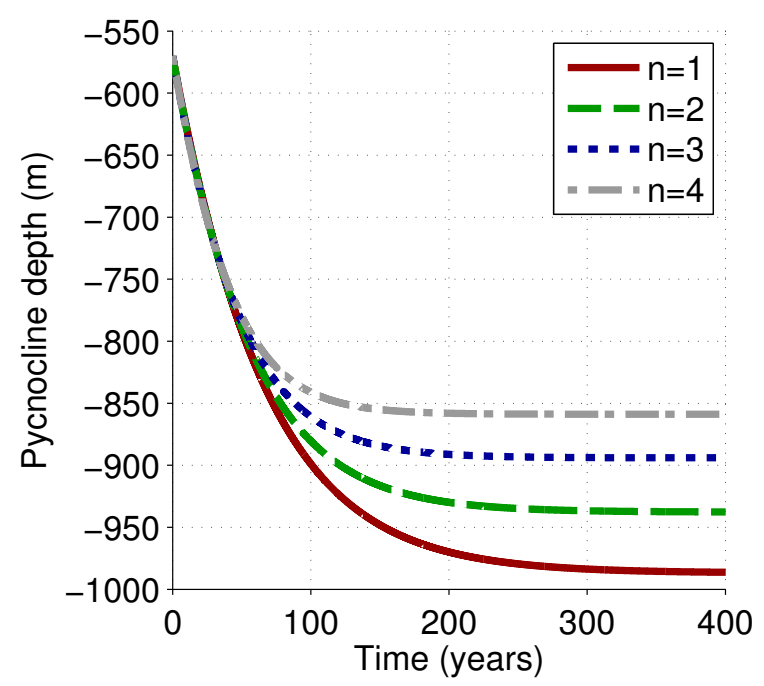

FIG. 2.4. Time series of pycnocline depth $D$ for four different values of $n$. In this plot, $\tau=0.26 \mathrm{~Pa}$.

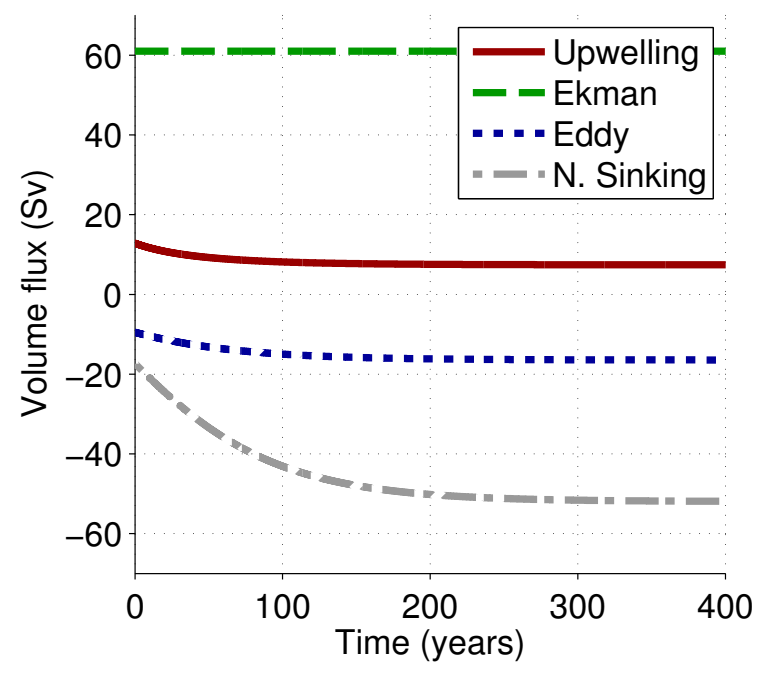

FIG. 2.5. Individual volume flux terms from the pycnocline evolution equation. Signs are assigned to each term based on the tendency for a term to deepen (positive) or shoal (negative) the pycnocline. 


\subsubsection{Stability}

We can use a one-dimensional phase portrait to investigate the stability of the equilibrium solutions of 2.12. We know from Descartes' sign rule that there is at most one positive root of 2.7, so there is only one physically realistic steady state. Assuming that at least one positive equilibrium point exists, we can see from 2.12 that this point must be stable. For arbitrarily small (and positive) values of $D$, the fourth term on the RHS of 2.12 dominates the sum, such that $d D / d t$ is positive. For arbitrarily large (and positive) values of $D$, the first two terms of 2.12 dominate, which implies that $d D / d t$ is negative. Thus, arrows on the non-negative half of our phase portrait will always point towards the only positive, physically realistic equilibrium point, i.e. the physically realistic equilibrium point is stable.

\subsubsection{Linearized time-dependent model}

Equation 2.12 is nonlinear and somewhat cumbersome to work with. In this subsection we develop a linear model that closely approximates the behavior of the full nonlinear equation near the equilibrium points, i.e. when $D$ is not too far away from $D_{0}$. Consider a small perturbation in $D$ such that $D(t)=D_{0}+D^{\prime}(t)$, where $\left|D_{0}\right| \gg\left|D^{\prime}\right|$. Equation 2.12 is then

$$
\dot{D}^{\prime}=-C_{E}\left(D_{0}+D^{\prime}\right)^{n}-C_{N}\left(D_{0}+D^{\prime}\right)^{2}+C_{W}+\frac{C_{U}}{\left(D_{0}+D^{\prime}\right)} .
$$

The first term can be expanded using the binomial theorem,

$$
\left(D_{0}+D^{\prime}\right)^{n}=D_{0}^{n}+\sum_{k=0}^{n-1}\left(\begin{array}{l}
n \\
k
\end{array}\right) D_{0}^{k}\left(D^{\prime}\right)^{n-k},
$$

the second term contains the factor

$$
\left(D_{0}+D^{\prime}\right)^{2}=D_{0}^{2}+\left(D^{\prime}\right)^{2}+2 D_{0} D^{\prime}
$$

and the last term can be linearized by the binomial approximation,

$$
\left(D_{0}+D^{\prime}\right)^{-1}=D_{0}^{-1}\left(1+\frac{D^{\prime}}{D_{0}}\right)^{-1} \approx D_{0}^{-1}\left(1-\frac{D^{\prime}}{D_{0}}\right)
$$


Putting these expressions in 2.16, we get

$$
\dot{D}^{\prime} \approx\left(-C_{E} D_{0}^{n}-C_{N} D_{0}^{2}+C_{W}+\frac{C_{U}}{D_{0}}\right)-\frac{C_{U} D^{\prime}}{D_{0}^{2}}-2 C_{N} D_{0} D^{\prime}-n C_{E} D_{0}^{n-1} D^{\prime},
$$

where we have neglected all perturbations above first order. The terms in parentheses add to zero using the equilibrium pycnocline depth equation 2.7, which leaves us with

$$
\dot{D}^{\prime} \approx-\left(\frac{C_{U}}{D_{0}^{2}}+2 C_{N} D_{0}+n C_{E} D_{0}^{n-1}\right) D^{\prime}
$$

The above is a differential equation of the form $\dot{y}=-\alpha y$, which has the standard solution $y(t)=y_{0}+\exp (-\alpha t)$. For our model, this can be written

$$
D(t) \approx D_{0}+C e^{-\alpha t}
$$

where $\sigma=1 / \alpha$ is the e-folding adjustment timescale for the system to adjust to its equilibrium state $D_{0}$. After some manipulation, we can express the adjustment timescale as

$$
\sigma_{n}=\frac{A D_{0}}{T_{U}+2 T_{N}+n T_{e d d y}} .
$$

According to equation 2.18, the adjustment timescale is proportional to the volume of the pycnocline and inversely proportional to a linear combination of volume fluxes. Note that the equilibrium depth $D_{0}$ also depends on the volume fluxes, so the numerator and denominator are not independent of one another.

In Figure 2.6, we plot the e-folding timescale versus wind stress for several different values of $n$. As $n$ increases, the timescales get shorter, and they get shorter for increasing wind stress as well. Notice that, even across the wide range of parameters used here, all of the timescales are multi-decadal, which suggests that the vertical structure of the ocean is slow to adjust to a new equilibrium pycnocline depth after it has been shifted by a perturbation. The long timescale could possibly explain why we have not observed much of a shift in the tilt of Southern Ocean isopycnals, despite the decadal increase in wind stress. The ocean is sluggish to respond to changes in forcing, since its structure is set by volume fluxes all over the globe. 


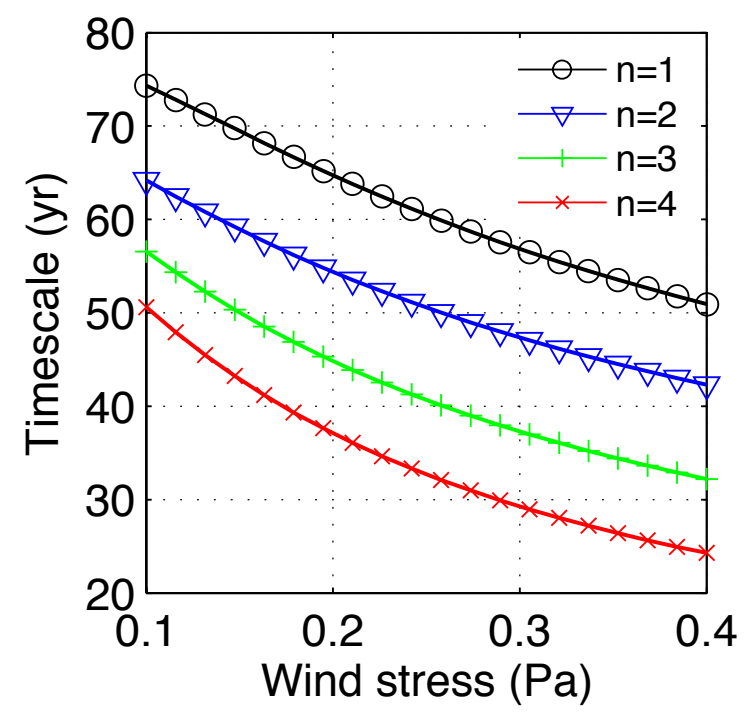

FIG. 2.6. Adjustment timescale predicted by the linearized time-dependent equation 2.18 for four different values of the eddy sensitivity parameter $n$.

\subsubsection{Limiting case: no deep water formation}

In this subsection, we explore certain (perhaps physically unrealistic) regimes in which the effect of some oceanic processes are neglected. If we turn off the formation of NADW $\left(T_{N}=0\right)$, equation 2.7 becomes

$$
D_{0}^{n+1}-b D_{0}-c=0
$$

which for the special case $n=1$ has the solution

$$
D_{0}=\frac{b+\sqrt{b^{2}+4 c}}{2},
$$

which gives us the rough proportionality $D_{0} \propto \tau$. For $n=2$, we can solve the corresponding cubic equation as before. The dynamical model is very similar to that produced for the general case, though the adjustment timescale is

$$
\sigma_{n}=\frac{A D_{0}}{T_{U}+n T_{e d d y}} .
$$

This limiting case may be relevant on sufficiently short timescales before the Southern Ocean is affected by adjustment with northern basins. 


\subsubsection{Limiting case: no net Southern Ocean flux}

If we instead turn off the entire Southern Ocean, with both $T_{\text {eddy }}$ and $T_{E k m a n}$ set to zero, equation 2.7 reduces to

$$
\frac{B_{U}}{D_{0}}=B_{N} D_{0}^{2},
$$

or

$$
D_{0}=\left(\frac{B_{U}}{B_{N}}\right)^{1 / 3}=\left(K_{v} A \frac{\beta L_{y}^{N}}{C g^{\prime}}\right)^{1 / 3},
$$

such that the pycnocline depth is set by the balance of low-latitude upwelling and northern sinking, as expected. The adjustment timescale goes to

$$
\sigma=\frac{A D_{0}}{T_{U}+2 T_{N}},
$$

where we have dropped the subscript $n$ since eddy processes have been neglected. If instead we just let $T_{e d d y}=T_{E k m a n}$, we get the condition

$$
D_{0}=\left(\frac{D_{r e f}^{n-1} L_{y}^{S} \tau}{\rho f A_{I}}\right)^{1 / n},
$$

which gives us the proportionality $D_{0} \propto \tau^{1 / n}$. Interestingly, the timescale of this adjustment takes the same form as 2.18, though it may have different values because of the above scaling.

\subsubsection{Limiting case: no low-latitude upwelling}

Now, if we arbitrarily turn off low-latitude upwelling, equation 2.7 becomes

$$
D_{0}^{n}+a D_{0}^{2}-b=0,
$$

which for the special case $n=1$ has the solution

$$
D_{0}=\frac{-1+\sqrt{1+4 a b}}{2 a},
$$

and the adjustment timescale goes to

$$
\sigma_{n}=\frac{A D_{0}}{2 T_{N}+n T_{\text {eddy }}} .
$$

This case may be relevant if the pycnocline adjustment is dominated by high-latitude fluxes. 


\subsection{DISCUSSION}

The simple box model used in this chapter is an expression of conservation of mass between the pycnocline and the abyss, with scaling relationships and parameters that appear to be reasonable for the global ocean. As such, it can only capture the gross, long-term features of the adjustment process. The real ocean is remarkably more complex, with continuous stratification, convection within the mixed layer, gyre circulation, and energetic eddies on many different scales. Nevertheless, the two-box model used here does generate three potentially useful predictions about the pycnocline adjustment process that may guide our thinking in the rest of this work:

1. Stronger SO wind stress should generally cause the pycnocline to deepen

2. The response time of the mean isopycnal slope to changes in wind stress is of decadal to centennial order

3. Both the magnitude and timescale of this response decrease with increasing nonlinearity of eddy-induced transport

In the following chapters, we test the above predictions in a more sophisticated numerical environment that includes representations of some of the processes neglected in the simple box model. 


\section{MODELING PHILOSOPHY AND GCM DESCRIPTION}

In this chapter, we discuss the modeling approach used in this work. We describe the model equations and boundary conditions used in our numerical experiments and attempt to make some qualitative predictions using the fundamentals of ocean circulation theory. First, we briefly examine the conceptual underpinnings of our approach to ocean modeling and outline some of the major challenges in the field of large-scale fluid dynamics simulation.

\subsection{ASPECTS OF CLIMATE MODELING}

Earth's climate system is fantastically complex. As we attempt to understand it, we encounter phenomena on a wide range of spatial and temporal scales, from rapid molecular motion in fluid boundary layers $\left(10^{-3} \mathrm{~m}, 10^{-9} \mathrm{~s}\right)$ to much slower geological processes that may involve the entire surface of the planet $\left(10^{7} \mathrm{~m}, 10^{15} \mathrm{~s}\right)$ and many in between. Often, processes operating on different scales interact with each other. These cross-scale interactions are important in fluid dynamical turbulence, for instance, where nonlinear coupling allows for transfers of energy and other properties between large and small features present in the fluid flow. Here we might think of the generation of midlatitude mesoscale storms through largescale instabilities in the jet stream. Neither our theoretical understanding nor our most sophisticated and powerful climate models can bear the weight of the full range of scales required for a complete characterization of the climate system, so we must treat multi-scale problems with ingenuity and care. One approach is to construct a collection of models that represent the system in question with varying degrees of sophistication and study the similarities and differences between models in the collection. 


\subsubsection{Model hierarchies}

The goal of theoretical science is to create, test, and improve models that allow us to better describe and understand the natural world. To construct such a model, we typically restrict our attention to important features of the system in question and disregard any unnecessary details. It is often difficult to unambiguously separate the "important" features from the unnecessary ones, though the desired order of accuracy can sometimes help us decide which details can be safely ignored.

For example, consider the gravitational potential $\Phi=\Phi(r, \phi, \lambda)$ of the Earth, which is determined by the distribution of mass throughout the planet's interior and on its surface. We can write down a mathematical description of the potential,

$$
\Phi_{N}=-\frac{G M}{r}\left(1+\sum_{n=2}^{N}\left(\frac{a}{r}\right)^{n} \sum_{m=0}^{n} P_{n m}[\sin (\phi)]\left[C_{n m} \cos (m \lambda)+S_{n m} \sin (m \lambda)\right]\right)
$$

where $G$ is the gravitational constant, $M$ is the mass of the Earth, $r$ is the distance from Earth's center, and $a, P_{n m}, C_{n m}$ and $S_{n m}$ are empirically determined constants. The complexity of equation 3.1 increases with $N$. For first order accuracy, we can simply treat the Earth as a point mass and retain the first term in 3.1 , such that $\Phi_{N} \approx-G M / r$. The simplicity of the point mass model makes it easy to work with and think about, and for many applications the additional details provided by extra terms are of negligible importance. Additional details add complexity to the model. For example, to include the oblate shape of the Earth in our description, we retain additional terms, increasing both the accuracy and the sophistication of our model. To represent continents and ocean basins we require additional terms still, and the inclusion of smaller scale features (e.g. mountains, ice sheets) in our model demands even higher $N$. Many of these details may be important to those whose job it is to keep satellites in orbit, since the cumulative effects of many small gravitational perturbations can alter a satellite's trajectory. The acceptable level of error between the model and observations guides our choice of $N$. 
The set of functions $\left\{\Phi_{N}\right\}$ arranged in order of increasing $N$ roughly constitutes a model hierarchy, as described in Held (2005). At the base of such a hierarchy is the simplest possible model that includes the major features of the system in question. It lacks detail, but it is easier to understand and completely characterize than more complex representations of the system. By examining what changes occur as $N$ increases, we learn about the effects brought about by higher-order terms. We might say that $N$ increases the complexity of the model, though not necessarily the accuracy.

In this work, the base of our hierarchy is the observationally-motivated mass balance model of Chapter 2. It only includes two density classes and a parameterized representation of the major conversion pathways between those two layers (e.g. Northern sinking, lowlatitude upwelling), and the only variable spatial structure built into it is the thickness of the pycnocline. It does not include gyre circulation, and it cannot in its current form represent coupled atmosphere-ocean interactions. Despite its rather severe simplicity, the conceptual model discussed in Chapter 2 offers a compelling view of ocean circulation and suggests a few interesting hypotheses (discussed at the end of Chapter 2) that may be tested at higher levels of our model hierarchy, specifically by using the primitive equation models considered in Chapters 4 and beyond. Primitive equation models include much more detailed and realistic representations of spatial structure, air-sea fluxes of buoyancy and momentum, forcing, and eddies. As such, they behave more realistically, but their behavior is harder to interpret.

\subsubsection{Eddies and the closure problem}

In the Southern Ocean and midlatitude atmosphere, a significant portion of meridional transport is accomplished by mesoscale eddies. Eddies can generally be thought of as departures from average values, i.e. $\phi^{\prime}(\mathbf{r}, t)=\phi(\mathbf{r}, t)-\bar{\phi}(\mathbf{r}, t)$, where $\overline{(\ldots)}$ is an averaging operator in time and/or space and $\phi(\mathbf{r}, t)$ is a state variable (e.g. temperature, salinity, momentum). 
The physical interpretation of what an eddy represents depends on the spatial and temporal scale of the averaging process. For instance, rings and meanders in the gulf stream are considered baroclinic eddies with a scale of a few hundred kilometers and a temporal scale of a few weeks. Eddies in the ocean tend to be smaller and longer-lived than large baroclinic eddies in the atmosphere.

In both analytical and numerical treatments of fluid mechanics, we always work with an averaged set of equations, and thus some sub-averaging scale features of the fluid flow (i.e. eddies) are filtered out (Salmon, 1998). However, under certain conditions (i.e. when there is a nonzero covariance between two eddy fields), the averaged-out fields may actually alter the mean flow. The potential importance of processes below the chosen averaging scale is the basis of the closure problem, which asks "can we express the effects of eddies on the mean flow in terms of the mean flow variables?" To put it another way, we might ask, does the function $\overline{v^{\prime} \phi^{\prime}}=f(\bar{v}, \bar{\phi})$ exist? If so, what form does it take? Attempts thus far to solve the closure problem have always involved additional assumptions not present in the fundamental equations of fluid mechanics. It may be the case that a general, closed-form solution derived purely from the Navier-Stokes equations does not exist.

Some practical progress has been made on the closure problem in geophysical fluid dynamics. The most common treatment of the problem is to assume that the eddy bolus velocity is a function of isopycnal slope, since eddies can get their energy from the release of potential energy stored in the tilt of surfaces of constant density (Gent and Mcwilliams, 1990). For a review of the historical development of eddy parameterization in numerical general circulation models, see Gent (2011). A discussion of the eddy parameterization scheme used in our implementation of MITgcm is given in 3.2.6. 


\section{$3.2 \quad$ PRIMITIVE EQUATION MODEL DESCRIPTION}

In this section, we summarize the essential features of the numerical model used in the remainder of this work, namely the Massachusetts Institute of Technology General Circulation Model (MITgcm). For a more detailed description of MITgcm, see Marshall et al. (1997a), Marshall et al. (1997b), and the user's manual (Adcroft et al., 2010). The source code is freely available online for anyone to download, modify, compile, and implement.

\subsubsection{Model equations}

The state of the fluid is described by a velocity field $\mathbf{v}=\mathbf{v}(x, y, z, t)$, a pressure field $P=P(x, y, z, t)$, and potential temperature $\theta=\theta(x, y, z, t)$. The density $\rho$ of the fluid can be expressed as a function of pressure and potential temperature using the equation of state of seawater. The model equations are:

$$
\begin{aligned}
D_{t} \mathbf{v}+(\mathbf{f} \times \mathbf{v})+\nabla_{z} P & =\mathbf{F} \\
\nabla_{z} \cdot \mathbf{v}+\partial_{z} w & =0 \\
g \rho+\partial_{z} P & =0 \\
D_{t} \theta & =Q \\
\partial_{t} \eta+\nabla \cdot(\eta+H) \mathbf{v} & =0 \\
\rho & =\rho(\theta, P, z),
\end{aligned}
$$

where $\mathbf{f}$ is the Coriolis parameter, $\eta$ is the height of the sea surface, and $H$ is a reference height. The operator $D_{t}=\partial_{t}+\mathbf{v} \cdot \nabla$ is the derivative following the motion of a fluid parcel, and the gradient operator is $\nabla=\nabla_{z}+\hat{k} \partial_{r}$, where $\nabla_{z}$ is the horizontal component and $\hat{k} \partial_{r}$ is the vertical component. The forcing and dissipation term $\mathbf{F}$ and the diabatic heating term $Q$ are specified separately by physics and forcing packages. In the ocean, these nonconservative terms are typically most important at the surface, where there can be a significant exchange 
of momentum and buoyancy with the overlying atmosphere. It is usually reasonable to treat the interior ocean as adiabatic (i.e. $Q=0$ ). We neglect salinity in our implementation of MITgcm in order to achieve faster model integration. This simplification does make the model somewhat less realistic, but we suspect that our results will not be drastically affected.

\subsubsection{Kinematic boundary conditions}

At the ocean bottom, we take $\dot{z}=0$ at $z=-H(x, y)$, so there is no flow into or out of the bathymetry. At the surface, we take $\dot{r}=D \eta / D t$ at $r=\eta$. The sea surface is free to move. In the horizontal, we take $\mathbf{v} \cdot \mathbf{n}=0$, where $\mathbf{n}$ is a unit vector that is perpendicular to the topography.

\subsubsection{Discretization}

Our implementation of MITgcm uses a C-grid scheme; the velocity components are defined at the edges of each grid cell. In the vertical direction, the sea surface height makes small deviations $\eta$ (around $0.1 \%$ of the ocean depth) above and below a reference height $H$. Grid cells may be partially or completely filled with "land" where the fluid cannot penetrate (Figure 3.1). The model field $h F a c$ gives the fraction of a grid cell that is filled with water; spatial integrations (e.g. zonal averages) must be weighted by this factor. Note that the grid

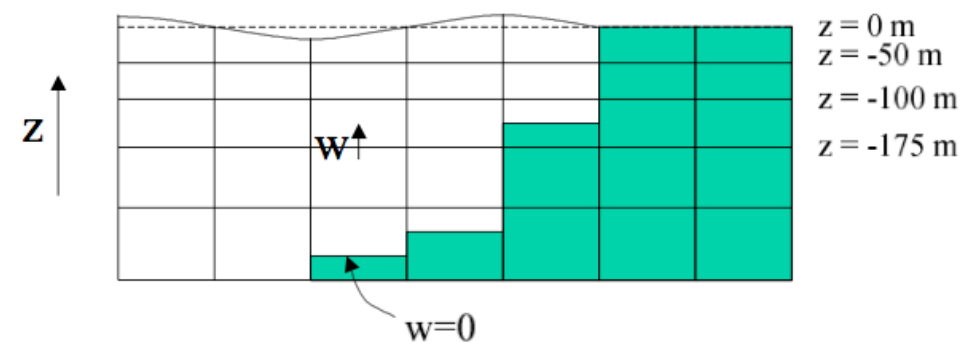

FIG. 3.1. Vertical coordinate $z$ and kinematic boundary conditions used in MITgcm. Figure from Adcroft et al. (2010). 
cells typically have uneven spacing in the vertical. This allows for finer vertical resolution near the surface where properties change quickly with depth and coarser vertical resolution in the relatively stagnant deep ocean.

\subsubsection{Solution procedure}

A two-dimensional elliptic equation is first solved to find the surface pressure and the hydrostatic pressure at each level using the weight of the fluid above (Adcroft et al., 2010). The momentum equation 3.2 is stepped forward in time (using an Adams-Bashforth scheme) and $w$ is computed from the continuity equation 3.3. It is possible to run the model in a nonhydrostatic mode that uses a different solution procedure, but for large-scale flow without strong vertical motion hydrostatic balance is typically an excellent approximation. All model integrations in this work assume hydrostatic balance.

\subsubsection{Dissipation}

Momentum dissipation is achieved using Laplacian and bi-harmonic friction:

$$
D_{V}=A_{h} \nabla_{z}^{2} v+A_{v} \frac{\partial^{2} v}{\partial z^{2}}+A_{4} \nabla_{z}^{4} v
$$

In the coarse resolution simulations, $A_{v}=10^{-3} \mathrm{~m}^{2} / \mathrm{s}, A_{h}=5.0 \times 10^{4} \mathrm{~m}^{2} / \mathrm{s}$, and $A_{4}=0.0$ $\mathrm{m}^{4} / \mathrm{s}$. In the eddy-permitting runs, sub-grid scale eddy viscosity is still parameterized, with coefficients $A_{v}=10^{-4} \mathrm{~m}^{2} / \mathrm{s}, A_{h}=10 \mathrm{~m}^{2} / \mathrm{s}$, and $A_{4}=10^{10} \mathrm{~m}^{4} / \mathrm{s}$. Observations of the global ocean suggest that horizontal mixing is typically many orders of magnitude stronger than vertical mixing, though as we will discuss in a later section it may prove more illuminating to decompose mixing into along-isopycal and cross-isopycnal components. 


\subsubsection{Tracer mixing}

For tracers, the diffusion tensor can be non-diagonal and have varying coefficients.

$$
D_{T}=\nabla \cdot(\mathbf{K} \nabla T)+K_{4} \nabla_{z}^{4} T
$$

where $\mathbf{K}$ is the diffusion tensor and $K_{4}$ is the horizontal coefficient for biharmonic diffusion. In the simplest possible case, subgrid-scale fluxes of heat are parameterized with constant horizontal and vertical diffusion coefficients. In this case, $\mathbf{K}=\operatorname{diag}\left(K_{h}, K_{h}, K_{v}\right)$, where the horizontal $K_{h}$ and vertical $K_{v}$ diffusion coefficients are constant but not equal to one another. Observations suggest that $K_{h} \gg K_{v}$.

In our implementation of MITgcm we use a somewhat more sophisticated eddy parameterization scheme (via the GMREDI package) in order to better represent the mixing of temperature by baroclinic eddies. There are two parts to this scheme, specifically eddy diffusion (Redi) and eddy-induced transport (GM). The "Redi" part of the scheme involves diffusion along and across isopycnal surfaces by projecting the tracer gradient onto the local isopycnal surface:

$$
D_{T, \text { Redi }}=\nabla \cdot\left(\kappa_{\rho} \mathbf{K}_{\mathbf{R e d i}} \nabla T\right)
$$

where $\kappa_{\rho}$ is the along-isopycnal diffusivity and the diffusion tensor is

$$
\mathbf{K}_{\text {Redi }}=\left(\begin{array}{ccc}
1 & 0 & s_{x} \\
0 & 1 & s_{y} \\
s_{x} & s_{y} & |s|^{2}
\end{array}\right),
$$

where $s_{x}=-\partial_{x} \rho / \partial_{z} \rho$ and $s_{y}=-\partial_{y} \rho / \partial_{z} \rho$ are the components of the slope. The GentMcWilliams (GM) component of the scheme represents the eddy-driven advective transport using a bolus velocity $\mathbf{u}^{\star}$ (Gent and Mcwilliams, 1990). A term of the form $-\nabla \cdot\left(T \mathbf{u}^{\star}\right)$ is added to the right-hand side of the (potential) temperature tendency equation (3.5). Griffies (1998) noted that the bolus flux can be written as a skew-flux that produces fewer computational modes:

$$
\mathbf{u}^{\star} T=-\kappa_{G M} \mathbf{K}_{\mathbf{G M}} \nabla T,
$$


where $\kappa_{G M}$ is a diffusion coefficient and $\mathbf{K}_{\mathbf{G M}}$ is

$$
\mathbf{K}_{\mathrm{GM}}=\left(\begin{array}{ccc}
0 & 0 & -s_{x} \\
0 & 0 & -s_{y} \\
s_{x} & s_{y} & 0
\end{array}\right) .
$$

The combined eddy parameterization term takes the form

$$
D_{T}=\nabla \cdot\left[\left(\kappa_{\rho} \mathbf{K}_{\mathbf{R e d i}}+\kappa_{G M} \mathbf{K}_{\mathbf{G M}}\right) \nabla T\right] .
$$

The coefficient of vertical eddy diffusion of temperature is set to $K_{v T}=3 \times 10^{-5} \mathrm{~m}^{2} / \mathrm{s}$ in both the control and eddy-permitting runs. Bi-harmonic eddy diffusion of temperature is used to suppress numerical noise in the eddy-permitting run, where $K_{4 T}=10^{10} \mathrm{~m}^{4} / \mathrm{s}$.

\subsubsection{Vertical mixing}

The K-Profile Parameterization (KPP) package in MITgcm includes several different schemes designed to treat various aspects of vertical mixing both in the surface boundary layer and in the interior (Large et al., 1994). Mixing in the interior is a function of shear instability and wave activity, while mixing in the boundary layer is determined by a polynomial profile subject to constraints from similarity theory in turbulence (Adcroft et al., 2010). KPP tends to enhance vertical mixing via surface forcing by allowing the influence of the boundary layer to penetrate into the thermocline.

\subsection{MODEL BATHYMETRY AND SURFACE FORCING}

For most of the numerical experiments described in the following chapters, we use the model domain shown in Figure 3.2 (left panel). The bathymetry is an idealized representation of an interhemispheric ocean basin with sloping sidewalls that represent continental shelves on both the western and eastern edges (Lovenduski and Ito, 2009). Most of the basin 
is zonally blocked, with the exception of a $10^{\circ}$ wide opening from $50-40{ }^{\circ} \mathrm{S}$ that mimics the geometry of Drake Passage and permits the existence of a circumpolar current. This bathymetric configuration will henceforth be referred to as the sector configuration. Despite the appearance of Figure 3.2, the domain is a section of a sphere. We use periodic boundary conditions in the channel, which has the somewhat curious implication that our channel is only $60^{\circ}$ wide on a sphere and yet is continuous. We do not expect this geometrically ambiguous situation to significantly alter our results.

Wind stress. The model is forced at the surface with a sinusoidal zonal wind stress profile $\tau=\tau(y)$ shown in Figure 3.2 (middle panel). The forcing consists of highly simplified representations of both Northern and Southern Hemispheric winds, including tropical easterlies and prevailing westerly winds. No meridional wind stress is applied. The profile $\tau=\tau(y)$ forces subtropical gyres and a circumpolar current, which respond quite differently to changes in surface wind stress. In the Southern Hemisphere, the gyre (including its western boundary current) may interact with the circumpolar current through fluxes of heat and momentum.

Buoyancy. The surface temperature of the model is restored to the simple sinusoidal profile shown in Figure 3.2 (right panel) by Newtonian relaxation using a timescale of one month. No other buoyancy fluxes are imposed on the model. The restoring profile mimics the excess of downward visible radiation over outgoing infrared in the tropics and the deficit of incoming visible to outgoing infrared at the poles. The large-scale temperature gradient established by this restoring mechanism powers the general circulation of the ocean model; as in the real ocean, the excess heat deposited in the tropics must be exported from the tropics to the poles where it can ultimately be removed from the ocean by surface fluxes.

One advantage of the sector configuration is that it allows for dynamic adjustment of equatorial stratification across the hemispheres. That is, we expect changes in Southern Hemispheric winds to alter the buoyancy distribution in the Southern Hemisphere, but given 
a.

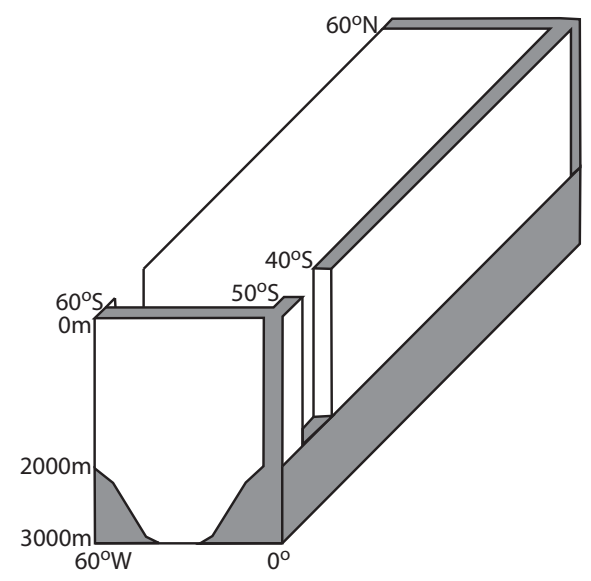

b.

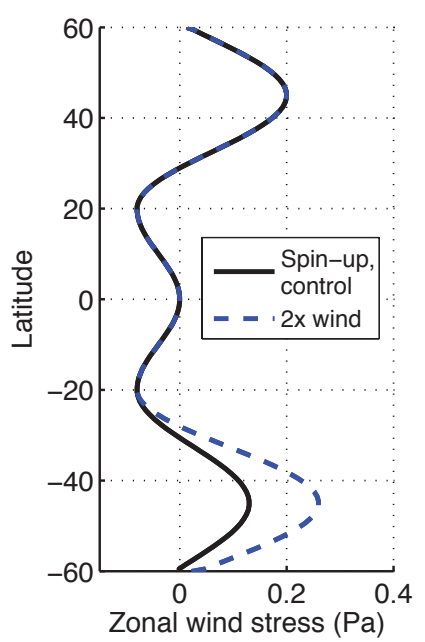

C.

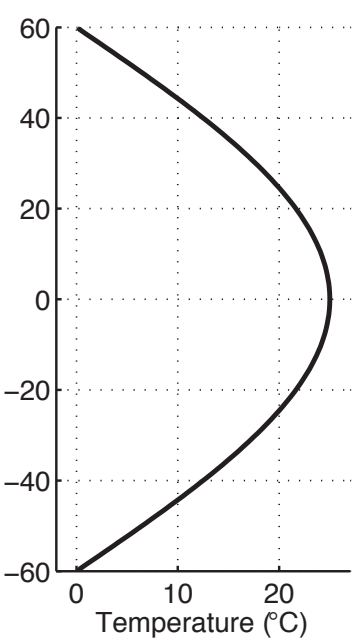

FIG. 3.2. Idealized model domain (left), wind stress forcing for the spin-up, control, and doubled-wind cases (middle), and the surface temperature restoring profile (right). Figure from Jones et al. (2011), supplementary information.

sufficient time those changes may be communicated through the tropics to the Northern Hemisphere, which itself may amplify or dampen the changes in the Southern Hemisphere via large-scale interactions (e.g. through the adjustment of the global pycnocline). The sector configuration can host long-term, interhemispheric adjustment processes that many other idealized modeling setups (e.g. a channel model with a sponge layer at the northern edge of the domain) cannot. However, the sector configuration is computationally expensive, as the temperature, pressure, and circulation fields in the tropics and Northern Hemisphere must be explicitly calculated.

In a steady-state, frictionless ocean in geostrophic balance, large-scale flow tends to follow contours of constant potential vorticity, i.e. lines of constant $f / h$ (here $f$ is the Coriolis parameter and $h$ is the thickness between two isopycnal surfaces). If we take $h$ to be the entire depth of the ocean for simplicity, we can plot surfaces of constant $f / h$ in our bathymetric configuration (Figure 3.3). The contours deviate from lines of constant latitude across the sills, as expected. As the water column gets thicker on the downstream side of the 
sill, the flow is constrained (by potential vorticity conservation) to deviate toward regions of larger $|f|$, i.e. towards the poles. The effect is reversed for flow on the upstream side of the sill. Eddy diffusion tends to be important where the lines of $f / h$ become very tightly packed, e.g. at high latitude and near the channel opening. Note that there are no contour lines of $f / h$ that circumnavigate the model domain, so eddy diffusion and stratification are both necessary for circumpolar flow in our sector configuration. Our model ocean is not actually uniform in density, so we should not expect flow to strictly follow contours of $f / h$. Nevertheless, potential vorticity conservation can help us understand, in a simplified way, how the current is influenced by topography.

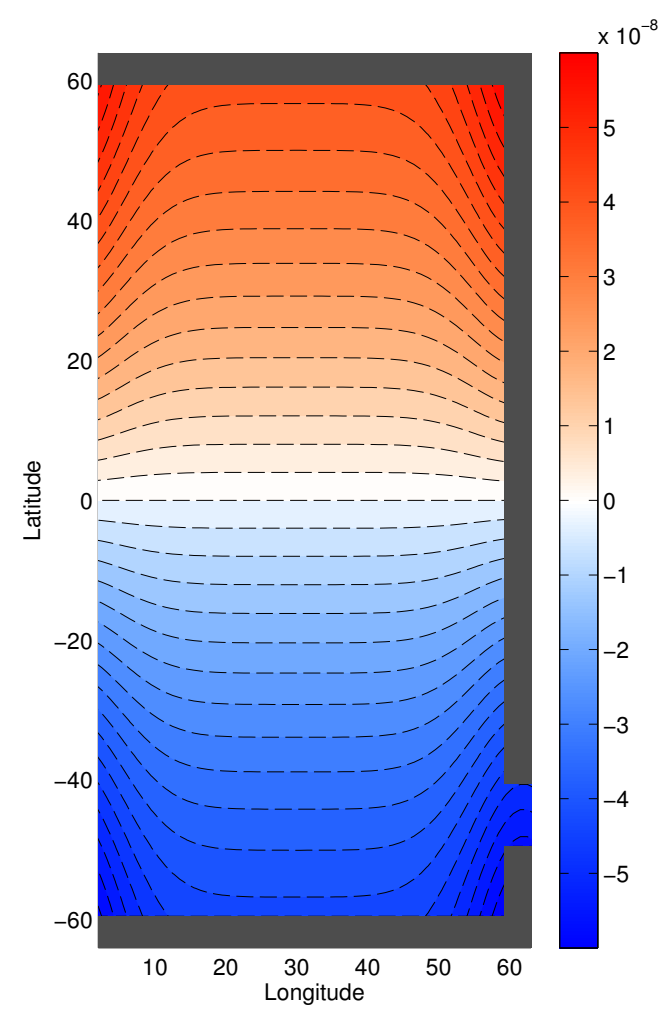

Fig. 3.3. Contours of $f / h$, where $h$ is taken to be the entire ocean depth. 


\subsection{STEP RESPONSE EXPERIMENTS}

In order to examine the transient response of the ocean's density structure and circulation to a change in surface wind stress, we perform numerical sensitivity experiments analogous to step response tests from control theory. Specifically, we instantaneously double the wind stress by switching from the "control" surface forcing profile to the "doubled wind" profile shown in Figure 3.2 (middle panel) and track the model's adjustment to its new equilibrium state. We purposefully use a step-function change instead of a gradual, more realistic ramp-up in order to get a clear adjustment signal. We run separate experiments using both coarse $\left(1^{\circ} \times 1^{\circ}\right)$ and eddy-permitting $\left(1 / 6^{\circ} \times 1 / 6^{\circ}\right)$ horizontal resolution in order to investigate the effect of eddying processes that are often parameterized in large-scale ocean models. In all of our experiments, we use 42 vertical levels spaced unevenly such that we have greater vertical resolution in the upper ocean, where temperature and circulation change rapidly with depth, and coarser vertical resolution in the deep ocean, which is relatively uniform and stagnant compared to the upper ocean. The change in thickness between any two adjacent grid cells is kept smaller than $20 \%$ in the surface ocean (top 100 m) and smaller than $10 \%$ in the deep ocean; this should discourage the formation of spurious computational modes that tend to arise at sharp transitions in resolution. Based on the predictions of Chapter 2, we expect both the circulation and density fields to respond across a wide range of timescales, from a few rotation periods to several centuries. Before we examine the results of specific numerical experiments, let us determine what qualitative predictions we can make using fundamental ocean circulation theory. This exercise should give us more insight into the behavior of the ocean model.

We calculate the Ekman (i.e. wind-induced) vertical velocity as the curl of the wind stress field (scaled by the Coriolis parameter $f$ ):

$$
w_{E k}=\frac{1}{\rho_{0}}\left[-\frac{\partial}{\partial y}\left(\frac{\tau_{x}}{f}\right)\right],
$$

where $\tau_{y}=0$ and $\rho_{0}$ is the reference density. In the interior ocean where the flow is weak, 
the depth-integrated Sverdrup velocity is given by

$$
\beta V=\rho_{0}^{-1}(\nabla \times \tau) \cdot \hat{\mathbf{k}}
$$

where $\beta$ is the meridional gradient of the Coriolis parameter (i.e. a geometric parameter that describes Earth's spherical shape and rotation rate). The depth-integrated streamfunction may be calculated using

$$
\Psi(x, y)=\frac{1}{\rho_{0} \beta} \int_{x_{e}}^{x}(\nabla \times \tau) \cdot \hat{\mathbf{k}} d x
$$

where $x_{e}$ is the eastern boundary. We can then calculate the depth-integrated zonal velocity components as $U=-\partial_{y} \Psi$ and $V=\partial_{x} \Psi$. We now apply these basic diagnostics to our model fields.

The control forcing profile sets up northward Ekman transport at the surface between 60 ${ }^{\circ} \mathrm{S}$ and $30{ }^{\circ} \mathrm{S}$ and southward Ekman transport between $30{ }^{\circ} \mathrm{S}$ and the equator. As such, there is a region of convergence where we expect to find Ekman pumping, northward Sverdrup flow, and a return flow along the western boundary. That is, we anticipate the presence of a subtropical gyre north of the channel latitudes. Note that the extrema of Ekman pumping/suction correspond to the extrema of wind stress curl by equation 3.8. The pattern of upwelling and downwelling is reversed in the Northern Hemisphere, where the dynamics outside of the tropics are dominated by the presence of two large gyres. South of $30{ }^{\circ} \mathrm{S}$, northward Ekman transport lowers the sea surface height (SSH) near the southern boundary and raises it on the equatorward side of the channel. The resulting meridional gradient in SSH supports the geostrophic component of the eastward-flowing circumpolar current.

By switching from the "control" profile to the "doubled wind" profile, we impose an instantaneous change in the wind stress and also the wind stress curl, which sets up changes in vertical motion within the top layer of the ocean (called the Ekman layer). Ekman pumping into the interior ocean is enhanced between $40{ }^{\circ} \mathrm{S}$ and $20{ }^{\circ} \mathrm{S}$, and there is stronger Ekman suction south of $40{ }^{\circ} \mathrm{S}$ (see Figure 3.4). The Taylor column model from fundamental GFD predicts that the depth-integrated velocity should be northward on the equatorward 

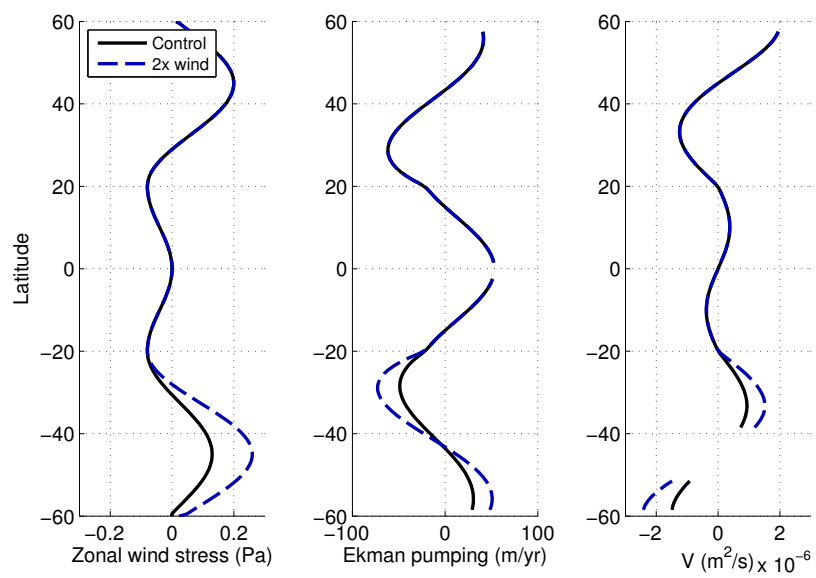

Fig. 3.4. Theoretical solutions given the wind stress profile. The Sverdrup velocity $V$ is not defined in the channel, so it is not shown.

side of the channel and southward on the poleward side of the channel (see equation 3.9). Sverdrup dynamics do not apply in the channel, so we do not calculate the depth-integrated velocity there. The subtropical gyre gets stronger under enhanced wind conditions, and if the dynamics in the subpolar region are Sverdrup-dominated there should be a gyre there as well. However, the flow at high southern latitudes may be dominated by strong cooling instead, such that water transported poleward at the surface loses buoyancy to the atmosphere and sinks into the interior ocean.

Enhanced Ekman pumping and suction steepens isopycnals across the channel by stretching them on the poleward side of the channel and compressing them on the equatorward side. Since tilted isopycnals are baroclinically unstable, we expect to find enhanced eddy activity in regions where the tilt is enhanced. The eddies will tend to flatten the isopycnal surfaces back out, until the tilt stabilizes in a new equilibrium state where wind-driven tilting effects balance eddy-driven flattening. The circumpolar current will adjust to any changes in tilt, since the current is in thermal wind balance with the isopycnal tilt. The theoretical circulation pattern is shown in Figure 3.5, where the direct wind-driven circulation is represented with straight arrows and the eddy flux is represented with wavy arrows. 
In the next several chapters, we compare the predictions made here on the basis of fundamental ocean circulation theory with the results of several numerical experiments. We begin with the coarse resolution configuration in the next chapter and discuss the eddypermitting variants afterwards.

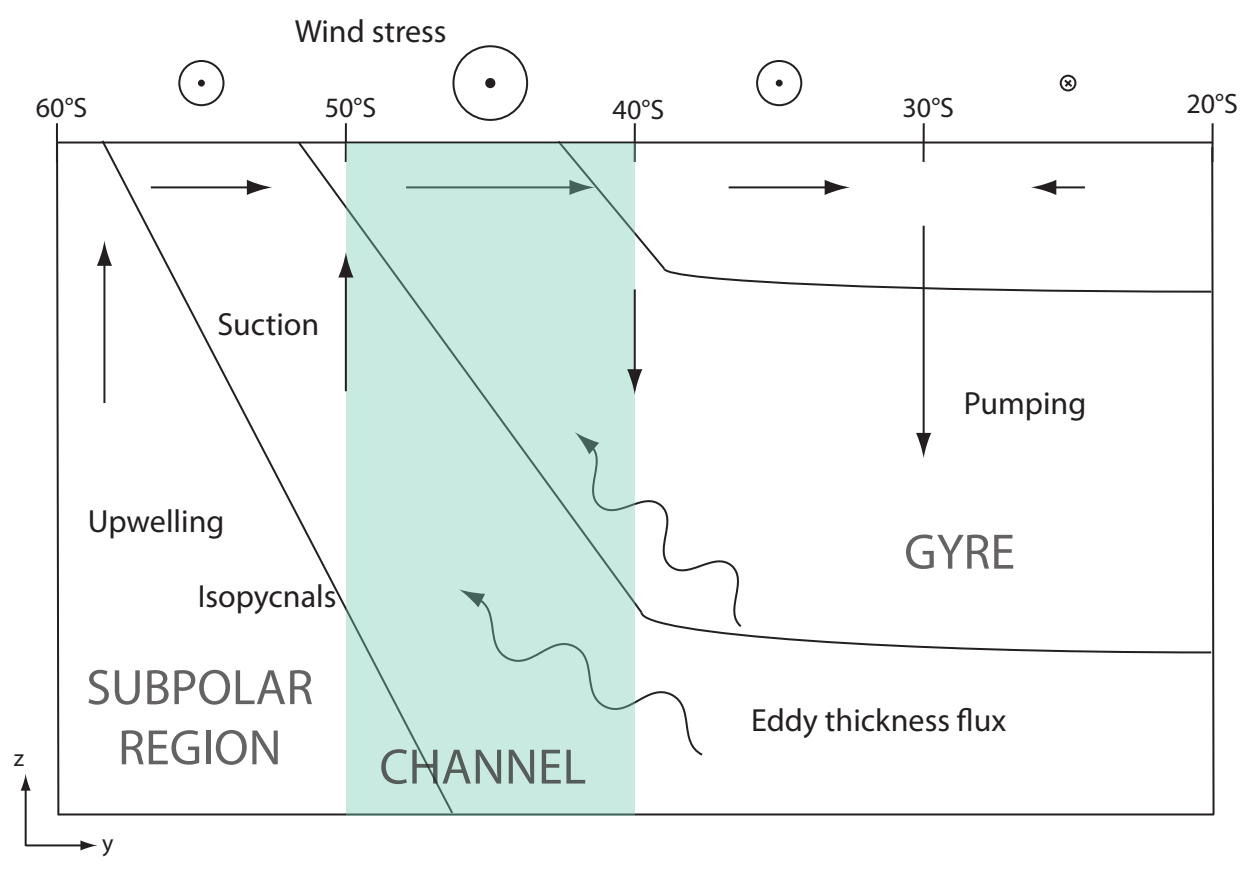

FIG. 3.5. Theoretical zonal-mean circulation in the sector model. Straight arrows indicate direct wind-driven circulation (i.e. Ekman transport at the surface and suction/pumping related to converges and divergences of the Ekman transport), and wavy arrows indicate the eddy flux in the interior. Straight arrows are scaled for doubled-wind conditions. The control circulation has the same pattern but is relatively weaker. 


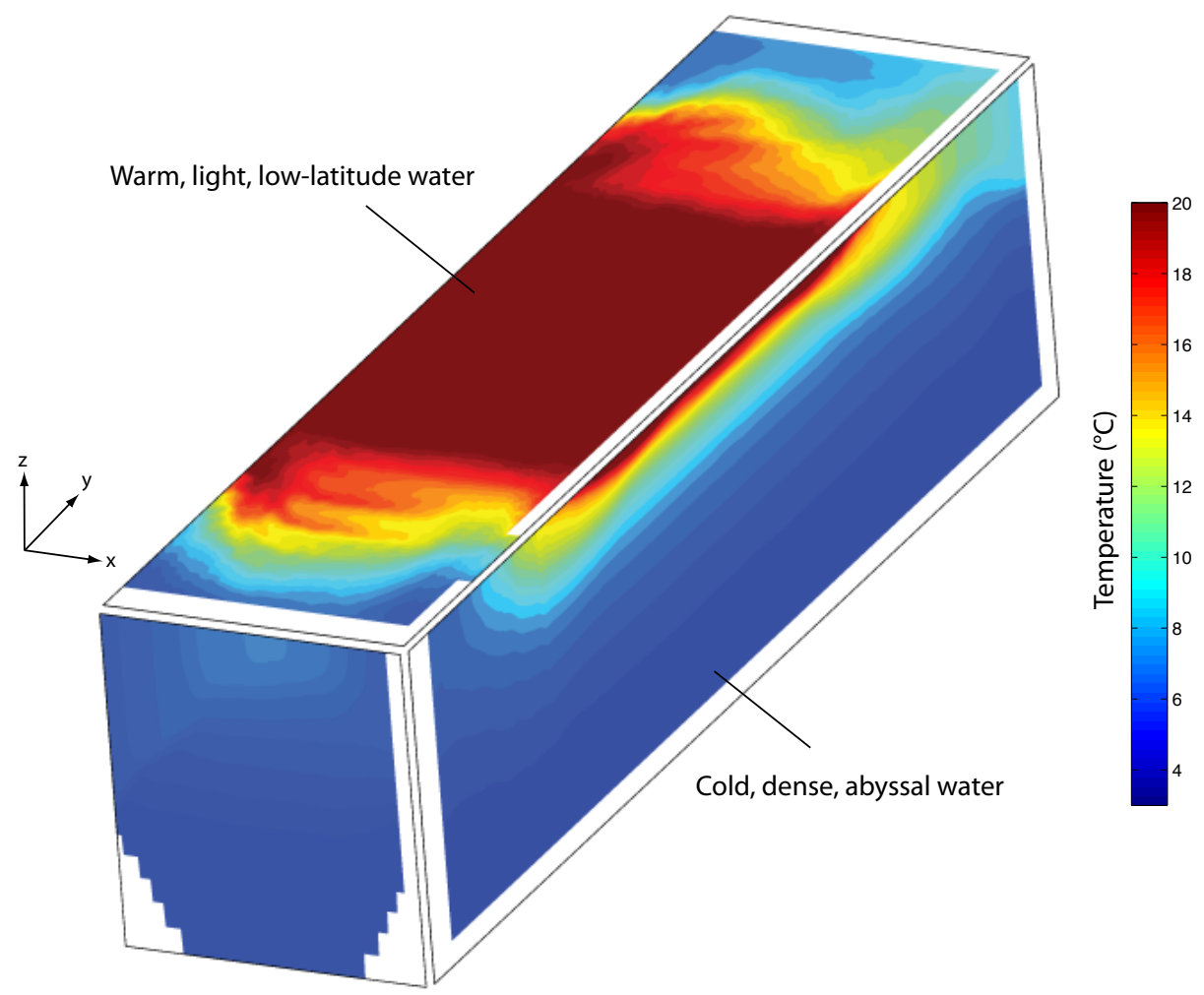

FIG. 3.6. Numerical realization of annual mean temperature with explicitly resolved mesoscale eddies. Note that the boundary is periodic only in the channel region; the eastern domain wall is omitted to better reveal the temperature structure. 


\section{COARSE RESOLUTION STEP RESPONSE EXPERIMENTS}

In Chapter 2, we hypothesized that the global ocean may take decades or centuries to equilibrate following a change in wind stress in the Southern Hemisphere. In this and the following chapters, we put this hypothesis to the test in a more sophisticated numerical environment. First, we begin with a primitive equation model in a sector configuration. The eddy-driven transport of buoyancy is parameterized using a Gent-McWilliams scheme as described in Chapter 3 (Gent and Mcwilliams, 1990). We subject the model to a step function change in wind stress profile $\tau=\tau(y)$ as described in Section 3.3 and examine both (i.) the differences between the model's initial and final state and (ii.) the model's approach to equilibrium (i.e. the transient response). We find that the model responds to the step change in forcing across a range of different timescales, including a multi-decadal adjustment involving a deepening of the global pycnocline as predicted by the simple model of Chapter 2.

\subsection{SURFACE FLUXES}

We begin our analysis at the air-sea interface. Note that we are essentially studying a one-way interaction; the atmospheric forcing does not vary in time (except for the instantaneous step-function change in wind stress) and receives no information from the oceanic model, so we have no way to represent any coupled air-sea interactions. In other words, changes in atmospheric forcing are communicated to the ocean through the surface, but the ocean does not alter the state of the atmosphere. However, since the net air-sea fluxes of momentum and buoyancy depend on both the forcing and the properties of the surface ocean, changes in surface ocean properties induce changes in flux. Changes in surface wind stress can alter both the momentum and heat fluxes by altering oceanic circulation. Let us begin by examining the net air-sea heat flux, both in the control and doubled-wind simulations. 


\subsubsection{Heat flux}

Model surface temperature is influenced by both transport and the net air-sea heat flux $H$, parameterized using a Newtonian relaxation scheme $H=-\lambda_{T}\left(T-T_{s}\right)$, where $T_{s}=T_{s}(y)$ is the prescribed surface temperature profile described in section 3.3 , and $1 / \lambda_{T}$ is the restoring timescale. We use the convention that $H>0$ corresponds to heat flux into the ocean. Right at the surface, the (potential) temperature equation is

$$
\frac{\partial T}{\partial t}+\mathbf{v} \cdot \nabla T=-\lambda_{T}\left(T-T_{s}\right)+G
$$

where the relaxation timescale $1 / \lambda_{T}$ is set equal to about one month, and $G$ represents the contribution from sub-grid scale fluxes and diffusion. Under steady-state conditions $\left(\partial_{t} T=0\right)$, transport and mixing must be in a balance with air-sea heat flux. For timescales much longer than $1 / \lambda_{T}$ (and assuming a steady state), we can use heat flux anomalies as a sort of "tracer" for transport differences between the doubled wind and control cases.

We compute the area-weighted mean heat flux $F=\int H d A / \int d A$, where the integration is performed over the ocean surface area within a selected band in latitude $\lambda$. We calculate $F$ for the global ocean (all latitudes), the Southern Hemisphere $\left(\lambda<0^{\circ}\right)$, the gyre region $\left(-40^{\circ}<\lambda<-30^{\circ}\right)$, and channel region $\left(-50^{\circ}<\lambda<-40^{\circ}\right)$. During the first year after the step change in forcing, the mean heat flux becomes less negative in both the gyre and channel regions, indicating that the zonal mean flux of heat from the ocean to the atmosphere is reduced. In both the Southern Hemispheric and global means, $F$ shows a positive linear trend, indicating that the ocean as a whole is taking up more heat from the atmosphere compared to the control case, despite the fact that the thermal forcing has not changed. A purely mechanical change in forcing can induce a change in air-sea buoyancy flux. The results for the first year are summarized in Table 4.1, and the spatial pattern of equilibrium net air-sea heat flux is shown in Figure 4.1. In both cases, the ocean absorbs heat from the atmosphere in tropical latitudes and releases it at middle and high latitudes. The imposed forcing establishes a temperature gradient which drives poleward heat transport. 
Table 4.1. Area-weighted mean surface heat flux $\left(\mathrm{W} / \mathrm{m}^{2}\right)$

\begin{tabular}{lccccc}
\hline & \multicolumn{2}{c}{ One year } & & \multicolumn{2}{c}{ Equilibrium } \\
\cline { 2 - 3 } \cline { 5 - 6 } Region & Control & $2 \mathrm{x}$ wind & & Control & $2 \mathrm{x}$ wind \\
\hline Channel & -3.6 & -1.3 & & -6.1 & -3.6 \\
Gyre & -9.4 & -7.1 & & -9.7 & -8.1 \\
S. Hem. & 2.3 & 3.1 & & 1.8 & 2.3 \\
Global & 1.7 & 2.1 & & 1.6 & 1.7 \\
\hline
\end{tabular}

The change in the spatial pattern of air-sea heat flux induced by stronger wind forcing (Figure 4.1, far right panel) reflects the action of two distinct mechanisms. In the subtropics, the gyre spins up - cold, high latitude water is exported equatorward at a faster rate on the eastern side of the domain, and warm, low latitude water is carried into the subtropics at a faster rate along the western edge of the domain. As a result, the ocean takes up more heat (or at least cools more slowly) in the eastern part of the gyre and cools at a faster rate to the west. In the channel and subpolar regions, the heat flux pattern reflects both the action of surface Ekman transport (cold water is transported towards the equator) and the shift of the mean position of the circumpolar current. The new mean position allows for warm, low latitude water to reach the southern edge of the domain at a faster rate. We discuss the standing pattern of the circumpolar current further in section 4.2.5.

\subsubsection{Momentum flux}

The momentum equation 3.2 is forced by the nonconservative term $\mathbf{F}$, which consists of a zonal component $F_{u}=\tau_{x} /\left(\rho_{0} \Delta z_{s}\right)$ and a meridional component $F_{v}=0$. Physically, $\mathbf{F}$ represents an acceleration driven by wind stress at the surface. The rate at which wind does work on the oceanic general circulation can be computed as $P=\left\langle\tau \cdot \mathbf{v}_{\mathbf{g}}\right\rangle$, where $\tau$ is the wind stress and $\mathbf{v}_{\mathbf{g}}$ is the geostrophic component of the surface velocity (Wunsch, 1998). Here we estimate the geostrophic velocity from the model sea surface height $\eta$ using the relationship 

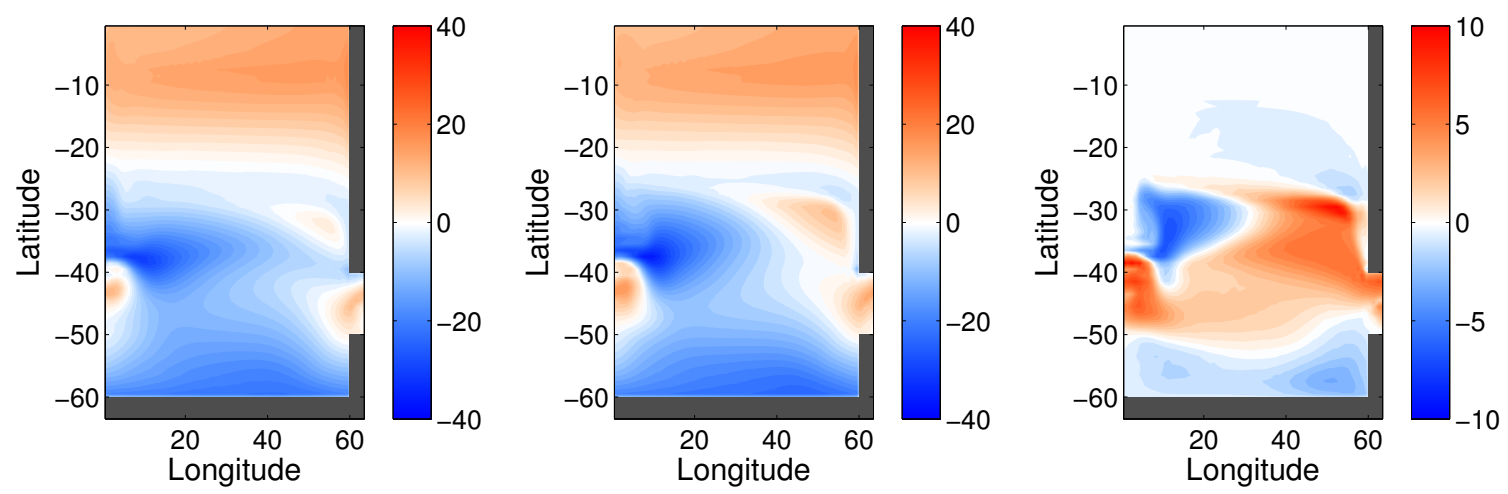

FIG. 4.1. Decadal average net air-sea heat flux approximately 3000 years into both the control (left) and doubled wind (middle) simulations $\left(\mathrm{W} / \mathrm{m}^{2}\right)$. Positive fluxes are directed into the ocean from the atmosphere. Also shown is the difference between the doubled wind and control cases (doubled wind minus control, right).

$\left(u_{g}, v_{g}\right)=\left(-g f^{-1} \partial_{y} \eta, g f^{-1} \partial_{x} \eta\right)$, and we take the wind stress from the prescribed forcing. Note that there is no forcing in the meridional direction, so only the zonal geostrophic velocity contributes to the work. Results are shown in Figure 4.2. The additional power from the stronger wind field mainly goes into the mean flow of the circumpolar current, especially near the channel opening where streamfunction contours are narrow.
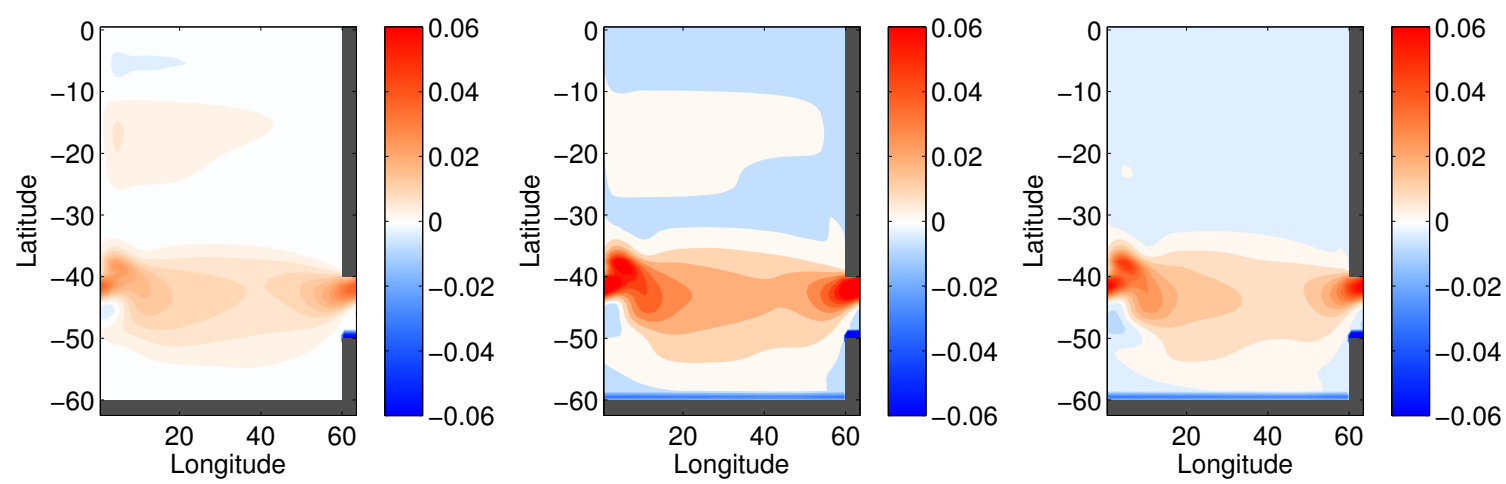

FIG. 4.2. Decadal average power input by the wind into the geostrophic component of the current flow $\left(\mathrm{W} / \mathrm{m}^{2}\right)$. Plotted are the control (left) and doubled wind (middle) cases as well as the difference between the doubled wind and control cases (right). 


\subsection{EQUILIBRIUM RESPONSE}

In this section, we compare the approximate steady states of the control and doubledwind experiments. We diagnose the steady state circulation and density fields using the sea surface height, isopycnal slope, zonal velocity, the meridional overturning streamfunction, the barotropic streamfunction, northward heat transport, and potential vorticity. We find that the fluid's equilibrium state is indeed sensitive to changes in wind stress, which is consistent with the behavior of other coarse resolution models with parameterized eddy transport of buoyancy (Hallberg and Gnanadesikan, 2001).

\subsubsection{Sea surface height}

As discussed in section 3.4, by switching from the control to doubled-wind profile, we induce a change in Ekman pumping (and therefore Sverdrup flow) that will spin up the subtropical gyre. We see evidence of subtropical gyre spin-up in Figure 4.3. Roughly between $-40^{\circ}<\lambda<-20^{\circ}$, more negative wind-stress curl leads to convergence at the surface, which enhances the sea surface height (SSH) and strengthens downward Ekman pumping in the region. The resulting enhanced Sverdrup flow returns along the western boundary, so we also see a stronger western boundary current associated with the subtropical gyre. In the Northern Hemisphere two gyres (both a subtropical and a subpolar gyre) are present. We will discuss the northern double-gyre system briefly in section 4.3.4.

In addition to spinning up the gyre, the change in wind forcing causes the circumpolar current to accelerate and shifts the configuration of its contours. Stronger northward Ekman transport enhances the existing meridional gradient of SSH by lowering the sea surface at high southern latitudes and raising it in the subtropics. The sea surface is depressed near the southern boundary, near our highly idealized representation of the Antarctic continent. The enhanced SSH gradient supports a stronger vertical shear of the circumpolar current through 

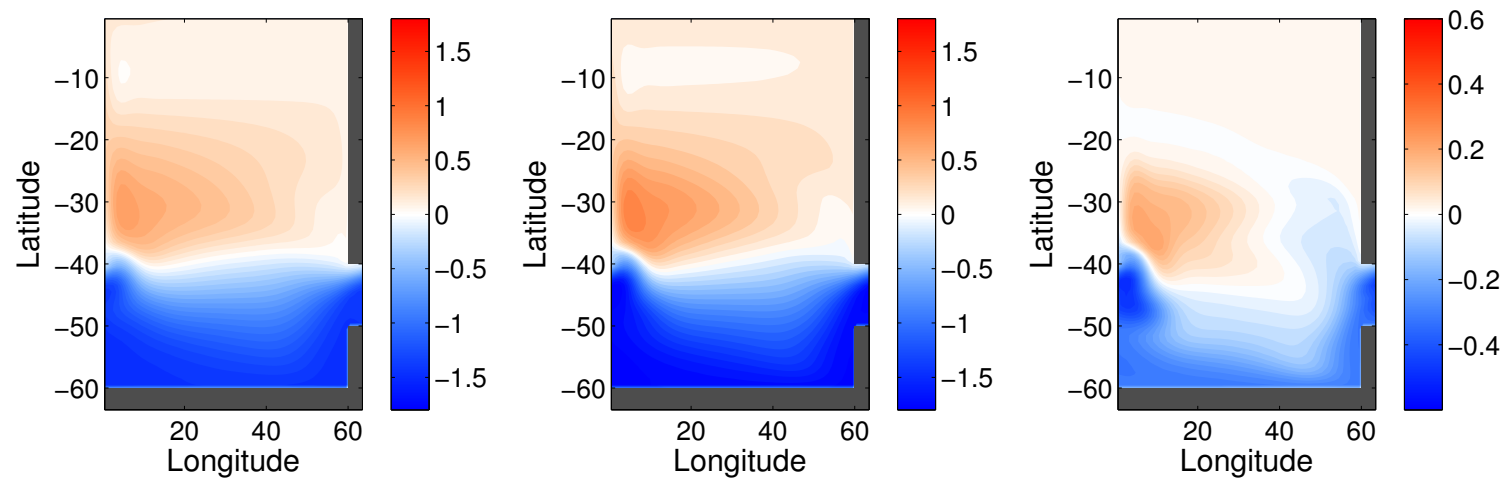

FIG. 4.3. Approximate decadal-mean steady-state (about 3000 years after the step change is imposed) sea surface height $(\mathrm{m})$ from both the control (left) and doubled-wind (middle) experiments. Also shown is the anomalous (doubled-wind minus control) sea surface height (right).

thermal wind balance. Since sea surface height is coupled to the subsurface isopycnal slope, we expect by this simple argument to see a corresponding basin-scale change in isopycnal slope. We now examine the slope in order to check this line of reasoning.

\subsubsection{Isopycnal slope}

First, we describe the method used to assign a zonal mean slope $\overline{s_{\rho}}$ to every latitude and depth. A small change in zonal-mean buoyancy $\delta \bar{b}$ can be written as

$$
\delta \bar{b}=\frac{\partial \bar{b}}{\partial y} \delta y+\frac{\partial \bar{b}}{\partial z} \delta z
$$

where $y$ is meridional distance and $z$ is the depth. Along surfaces of constant buoyancy $(\delta \bar{b}=0)$, we can rearrange equation 4.2 to get

$$
\overline{s_{b}}=\frac{\delta z}{\delta y}=-\frac{\partial_{y} \bar{b}}{\partial_{z} \bar{b}}=-\frac{M^{2}}{N^{2}}
$$

where $\overline{s_{b}}$ is the slope of a surface of constant buoyancy, $M^{2}=\partial_{y} \bar{b}$ is the horizontal change in buoyancy, and $N^{2}=\partial_{z} \bar{b}$ is the vertical stratification. In our salinity-free simulation, surfaces of constant density are parallel to surfaces of constant buoyancy, so we can take $\overline{s_{b}}$ to be the isopycnal slope $\overline{s_{\rho}}$. We define isopycnal slope at each grid point using a centered finite-difference version of equation 4.3. 
Ekman suction south of the channel and Ekman pumping north of the channel help to maintain the tilt of zonal-mean isopycnal surfaces. The doubled-wind profile supports a much stronger (more negative) slope across the northern edge of the channel, as shown in Figure 4.4. At high southern latitudes, the doubled-wind profile sets up a more negative tilt through enhanced buoyancy flux and wind-driven upwelling of cold, deep waters. Though we do see a basin-scale increase in isopycnal tilt, it is interesting to note that this pattern is not spatially uniform; there is a region of flattening around $50{ }^{\circ} \mathrm{S}$, and the increase in tilt is particularly sharp on the northern flank of the channel. The increased tilt supports a strengthened surface-intensified jet, discussed further in the next section.
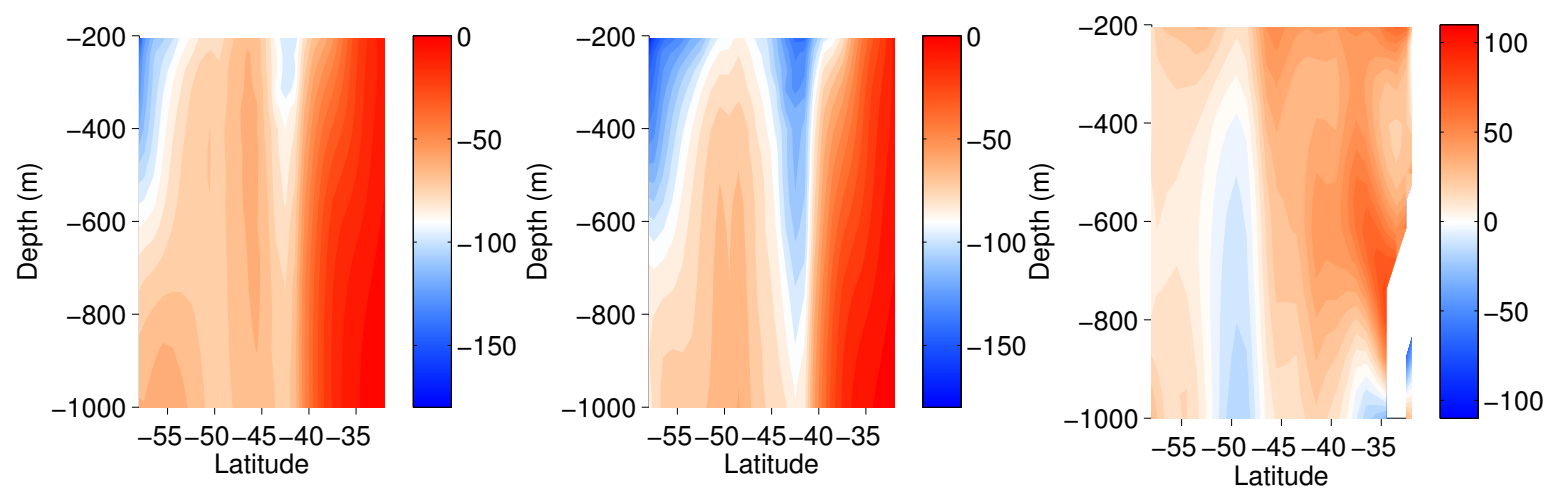

FIG. 4.4. Zonal mean approximate steady-state isopycnal slope $\overline{s_{\rho}}=-M^{2} / N^{2}$ (dimensionless) for the control (left) and doubled-wind (middle) coarse resolution experiments. Anomalous slope (doubled wind minus control, \% change) is shown in the far right panel. A negative slope corresponds to a surface that deepens with increasing latitude.

\subsubsection{Zonal mean jet}

The circumpolar current is in geostrophic balance with the large-scale meridional gradient in SSH. In Figure 4.5 we see that the doubled-wind profile drives a stronger surfaceintensified zonal mean jet near the northern flank of the channel (between $45^{\circ} \mathrm{S}$ and $40^{\circ} \mathrm{S}$ ). The mean zonal velocity is about $30 \%$ faster in the doubled wind case, with a $40 \%$ increase in the surface velocity. The equivalent depth $H_{0}$ (determined by fitting the velocity profile to the exponential $\left.v(z)=v(0) \exp \left(-z / H_{0}\right)\right)$ increases by about $24 \%$ from $550 \mathrm{~m}$ to $680 \mathrm{~m}$. 
The velocity shear of this idealized profile is given by $\partial_{z} v(z)=-\left(v(0) / H_{0}\right) \exp \left(-z / H_{0}\right)$, so an increase in $v(0)$ strengthens the shear while an increase in $H_{0}$ reduces the shear. The net result is a faster current with stronger vertical shear, since the increase in surface current overwhelms the increase in equivalent depth.
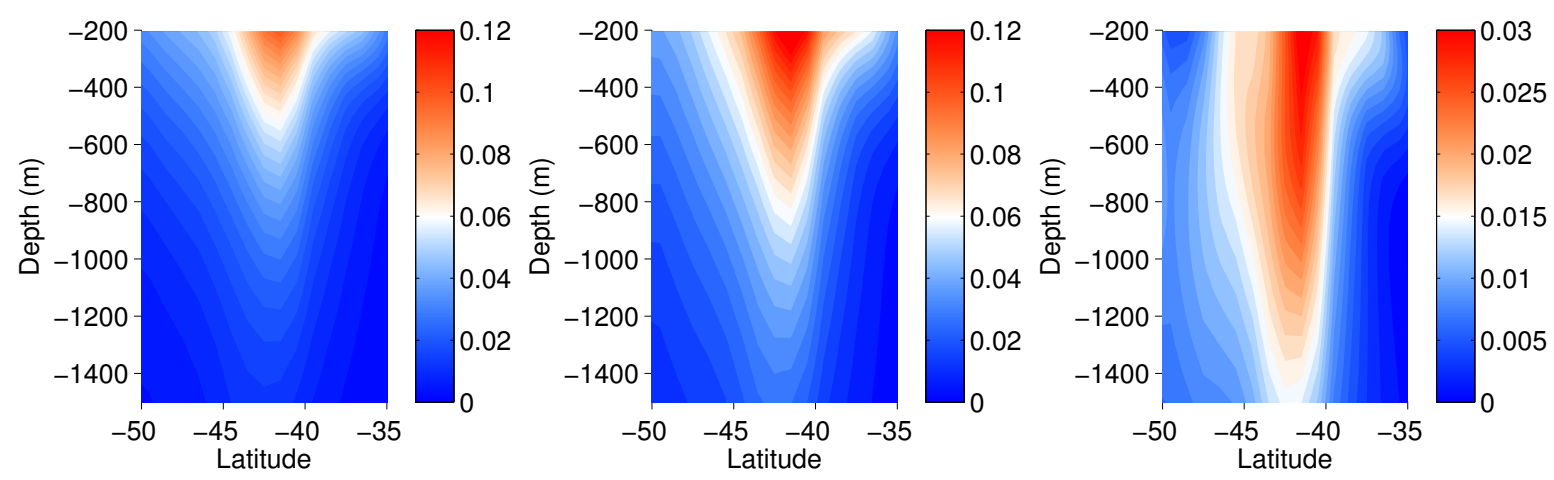

Fig. 4.5. Zonal mean approximate steady-state zonal velocity (m/s) for the control (left) and doubled-wind (middle) coarse resolution experiments. Anomalous slope (doubled wind minus control) is shown in the far right panel. Note that all values on this plot are positive.

\subsubsection{Meridional overturning circulation}

In this subsection we apply a version of residual mean theory to our model circulation fields (Andrews and McIntyre, 1976). Our goal is to examine the meridional overturning circulation in the Southern Hemisphere and understand how it responds to an increase in wind stress. The Eulerian mean streamfunction is given by

$$
\bar{\psi}(y, z)=-\int_{-H}^{z} \bar{v}\left(y, z^{\prime}\right) d z^{\prime}
$$

where the overbar denotes a time and zonal mean. We can roughly think of $\bar{\psi}$ as the winddriven overturning cell, since it is essentially set by (i.) Ekman transport, (ii.) the induced vertical motion caused by convergences and divergences in the surface layer, and by (iii.) a return circulation in the interior. The Eulerian mean circulation tends to steepen isopycnals in the interior. The eddy-induced streamfunction tends to flatten interior isopycnals and is 
given by

$$
\psi_{e d d y}(y, z)=\frac{\overline{v^{\prime} b^{\prime}}}{N^{2}}
$$

where $\overline{v^{\prime} b^{\prime}}$ is the eddy buoyancy flux and $N^{2}$ is the vertical stratification. If we use the closure $\overline{v^{\prime} b^{\prime}}=-K \overline{b_{y}}$, then the eddy-induced streamfunction becomes

$$
\psi_{e d d y}=-K \frac{\overline{b_{y}}}{\overline{b_{z}}}=K \overline{s_{\rho}},
$$

where we have used equation 4.3 in the last step. We diagnose $K$ directly from model output of the GM-REDI package described in section 3.2.6. Finally, we take the residual streamfunction to be the sum of the Eulerian mean and the eddy induced streamfunctions, $\psi^{\star}=\bar{\psi}+\psi_{e d d y}$.

Equilibrium streamfunctions are shown in Figure 4.6, with the control case plotted in the left column and the doubled-wind case shown in the right column. The average wind-driven circulation $\bar{\psi}$ increases by about $50 \%$ in the channel, and the maximum value $\max (\bar{\psi})$ in the channel increases by about $90 \%$. The circulation $\bar{\psi}$ flows equatorward at the surface (driven by Ekman transport), downwells along the northern edge of the channel, and upwells over a much larger area south of the jet core. The eddy induced circulation $\psi^{\prime}$ also gets stronger but with opposite sign. The contours of $\psi^{\prime}$ are most tightly packed in the upper $500 \mathrm{~m}$ of the ocean, and there is a clear circulation that is associated with the mean position of the zonal jet. Since the Eulerian mean and eddy-induced streamfunctions have different vertical structures, eddy compensation is not perfect and the residual circulation gets stronger with increased wind stress. This is consistent with what has been observed in other coarse resolution models with parameterized eddies (Hallberg and Gnanadesikan, 2001). The poleward circulation required by energy balance considerations is largely carried out by eddies in the top $50 \mathrm{~m}$ of the ocean, which are not shown in the figure. The surface eddy-driven circulation is around 4-5 times stronger than that seen in the interior, established by very steeply tilted isopycnals approaching their outcrop latitudes. 

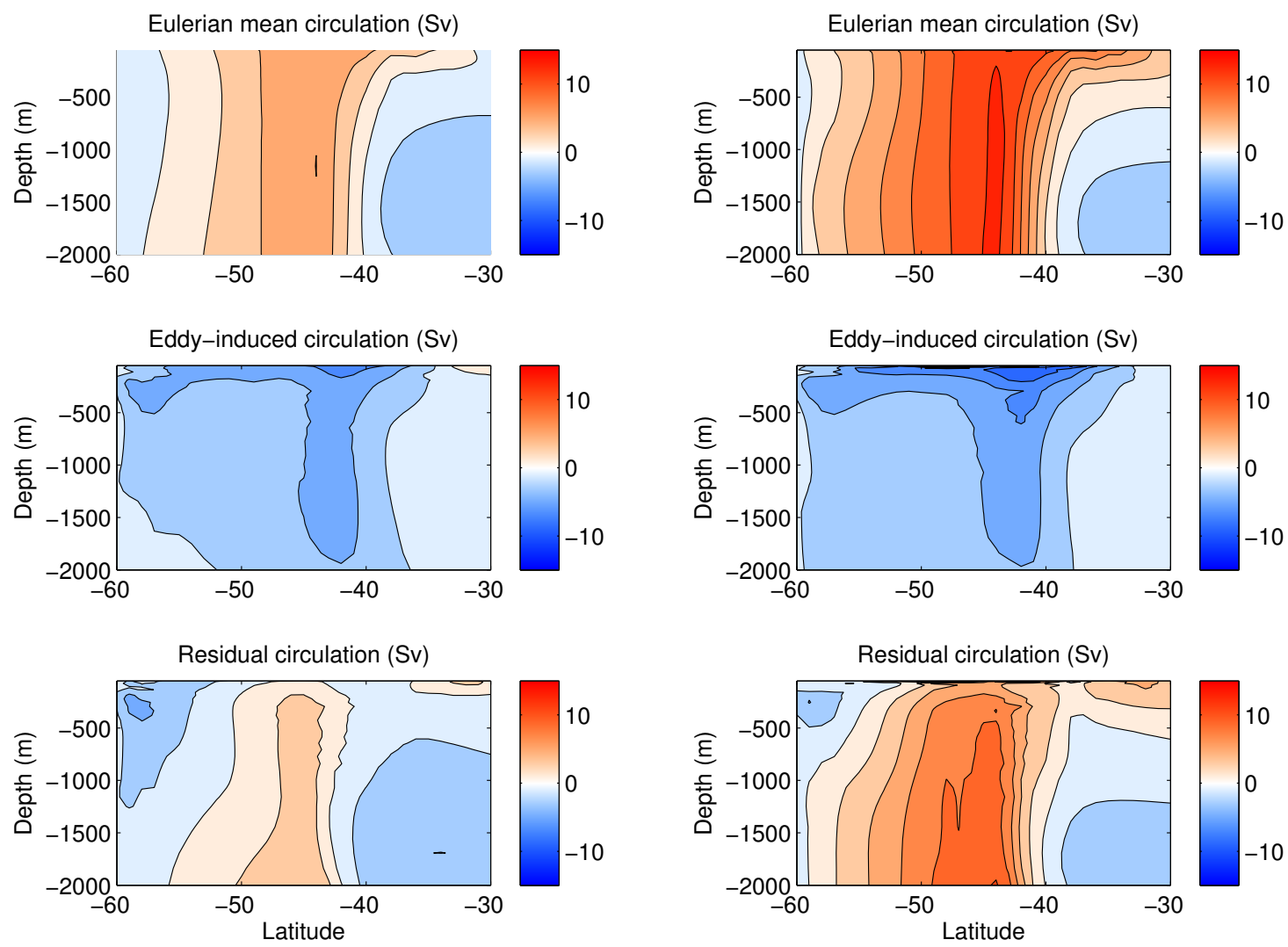

Fig. 4.6. Low resolution streamfunctions below $50 \mathrm{~m}$ and above the sill for both the control (left column) and doubled-wind (right colum) simulations. Shown are the Eulerian mean (top row), eddy-induced (middle row), and residual (bottom row) streamfunctions. Contours are down from -15 to 15 every $2 \mathrm{~Sv}$. 
One disadvantage of this approach is that depth-space overturning streamfunctions do not represent the net transport of water masses (Hallberg and Gnanadesikan, 2006). Since depth-space contours only track lines of constant volume transport, they may cross over isopycnal lines even when the rate of water mass transformation is very small (e.g. in the interior ocean in locations where mixing is weak). A more accurate approach is to describe the overturning circulation in potential density space (Marshall et al., 1997a). Constructing a potential density space streamfunction from z-coordinate model data (such as that generated by MITgcm) is certainly possible, but it requires frequent output of the position of buoyancy surfaces (ideally at each timestep, computational memory issues notwithstanding). A package for MITgcm (called layers) is now available that computes the density-space streamfunction at runtime (Abernathey et al., 2011). We leave the calculation of densityspace streamfunction contours for future work.

\subsubsection{Barotropic streamfunction}

The contours associated with the circumpolar current meander in the meridional direction as they cross topographic features and encounter variations in stratification. One method of visualizing these contours is to compute the depth-integrated zonal velocity,

$$
U(\phi, \lambda)=\int_{0}^{-H} u(\phi, \lambda, z) d z
$$

and the barotropic (depth-independent) streamfunction in spherical coordinates,

$$
\psi(\phi, \lambda)=\int_{0}^{\lambda} U\left(\phi, \lambda^{\prime}\right) a d \lambda^{\prime} .
$$

The barotropic streamfunctions for the control (left) and doubled wind (right) cases are shown in Figure 4.7. In response to the change in wind stress, the circumpolar current speeds up and meanders a little bit further northwards on the western side of the basin and shifts several degrees further southward across the middle of the basin. The enhanced meandering of circumpolar current contours reflects a change in the stationary pattern of 
the current, with implications for poleward heat transport (see section 4.2.6). We also see evidence of the spin-up of the subtropical gyre and associated western boundary current between $40{ }^{\circ} \mathrm{S}$ and $20{ }^{\circ} \mathrm{S}$. The circumpolar current speed-up and gyre intensification are consistent with predictions from our calculations in section 3.4, but we did not predict the change in the stationary wave pattern of the circumpolar current. Since barotropic flow is constrained to follow $f / h$ contours, the change in stationary wave pattern must be supported by changes in stratification and eddy mixing. It is interesting to note that we did not change the position of maximum wind stress, only its magnitude. The mean path of the circumpolar current is sensitive to the strength of wind stress forcing; it is not uniquely determined by the spatial distribution of wind stress extrema.
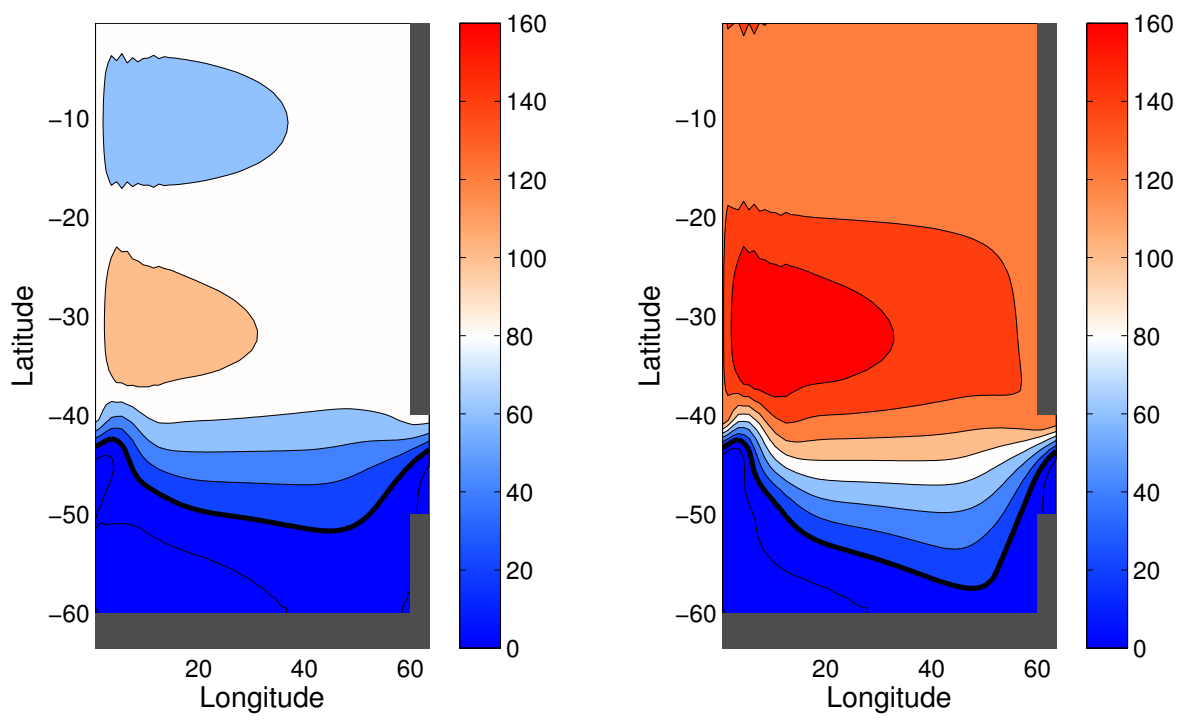

FIG. 4.7. Barotropic streamfunctions (Sv) for both the control (left) and doubled-wind (right) simulations. The $20 \mathrm{~Sv}$ contour is drawn in thick black for ease of comparison, and the contour interval is $20 \mathrm{~Sv}$. 


\subsubsection{Northward heat transport}

In general circulation theory, it is common practice to decompose the eddy fields into stationary and transient components. Stationary eddies can be roughly described as departures from a zonal mean state, while transient eddies are departures from a time-mean state. However, constructing the zonal mean state also involves establishing a time mean state that may, in general, be defined using a different temporal averaging period than that used to define transient eddies. Since we have a great deal of freedom in choosing the temporal scales of the two aforementioned averaging operators, the decomposition process is somewhat arbitrary. The results of the procedure are strongly dependent on the choice of the two temporal averaging scales used to define the zonal mean and time mean state. Recall that the physical interpretation of an eddy depends on the choice of spatial and temporal averaging scales.

In this section we discuss the decomposition procedure and analyze both transient and steady state snapshots of northward heat transport. The zonal mean state is defined by $\left[\bar{f}^{L}\right]$, where the overline represents a long-term time averaging operator of the form

$$
\bar{f}^{L}=\frac{1}{\Delta t} \int_{0}^{\Delta t} f d t
$$

and the bracket represents a zonal averaging operator

$$
\left[\bar{f}^{L}\right]=\frac{1}{W} \int_{x_{e}}^{x_{w}} \bar{f}^{L} d x,
$$

where $W$ is the basin width, $x_{e}$ is the longitude of the eastern boundary and $x_{w}$ is the longitude of the western boundary. The zonal average must be defined this way (instead of as an average over all longitudes as is common practice in the atmosphere) because of the presence of continental barriers in the ocean. The fluctuations associated with transient eddies appear as departures from the time mean state, i.e. $f^{\prime}=f-\bar{f}$, and the fluctuations associated with stationary eddies appear as departures from the zonal mean state, i.e. $\bar{f}^{\star}=$ 
$\bar{f}-\left[\bar{f}^{L}\right]$. The net northward transport of heat $[\overline{v T}]$ can be decomposed into contributions from the mean, stationary eddy, and transient eddy circulations,

$$
[\overline{v T}]=[\bar{v}][\bar{T}]+\left[\bar{v}^{\star} \bar{T}^{\star}\right]+\left[\overline{v^{\prime} T^{\prime}}\right]
$$

In our sector model configuration, the stationary eddies are associated with changes in the standing pattern of the gyre and circumpolar current, and the transient eddies are associated with episodic weather-like events in the ocean (eddy transport and mixing).

In the coarse resolution case presented here, the averaging period for both the zonal mean state $[\bar{f}]$ and the time mean state $\bar{f}$ is 10 years. Since the averaging periods are the same, most of the eddy contribution will show up in the "stationary" category. The transient contribution would be stronger if we used a longer averaging period to define the zonal mean state. We use different averaging periods for the fine-resolution results presented in the next two chapters, so care should be taken when comparing decomposition results between experiments. In any case, the sum of the stationary and transient components is the total "eddy" contribution to the heat flow, i.e. the portion of the heat flow not accounted for by the mean circulation.

In the control configuration, the net Southern Hemispheric heat transport is negative (poleward), and most of the transport is accomplished by a combination of the mean circulation and stationary eddies (see Figure 4.8). There is very little (but not zero) poleward transport across the channel $\left(50-40^{\circ} \mathrm{S}\right)$, as the mean and eddy components are in approximate balance. Five years after the step function change is applied (left column of Figure 4.8), we find an overall increase in poleward heat transport across both the channel and gyre regions. The wind-driven mean circulation is indeed stronger (and sharper) compared to the control case, but the stationary eddy component is actually larger than the mean circulation and causes heat transport to be poleward in the channel. We might say that the southward stationary eddy transport has overcompensated for the increase in mean northward transport. The transient eddies are nearly an order of magnitude smaller than the other terms, so 
the transient eddy heat flux is not clear on the plot. In equilibrium (right column of Figure
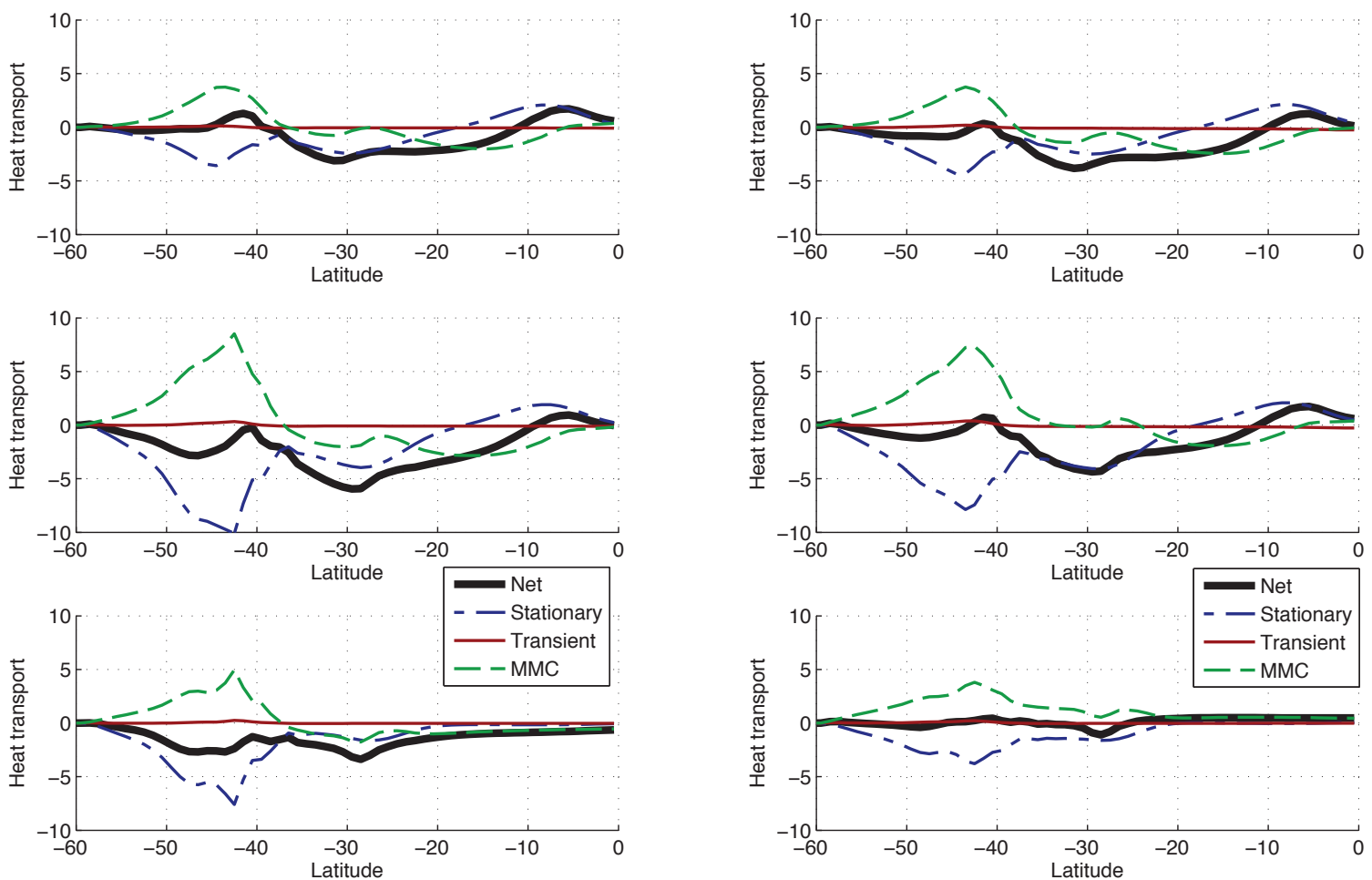

FIG. 4.8. Zonal mean northward heat transport, (left) annual mean 5 years after the step change is applied and (right) decadal average heat transport in approximate equilibrium, 3000 years after the perturbation is applied. The top row is the control case, the middle row is the doubled-wind case, and the bottom row is the difference (doubled wind minus control) between the two.

4.8), the stationary eddy component of the heat transport nearly cancels out the sustained increase in wind-driven transport at all latitudes. The compensation between wind-driven and eddy-driven cells appears to be fairly complete in this equilibrium picture. The change in net transport is very close to zero throughout the entire Southern Hemisphere. However, the transport shown in Figure 4.8 is the depth-integrated transport, so a value of zero is consistent with transport of opposite sign in the upper and lower oceans. Any regions of sustained baroclinic (i.e. not uniform with depth) warming/cooling in the doubled wind run may be supported by this kind of dipole in heat transport. We discuss the transient behavior 
of northward heat transport in section 4.3.2.

\subsubsection{Potential vorticity}

Potential vorticity (PV) is a scalar field that is conserved following large-scale, frictionless flow (Vallis, 2006). Since PV conservation offers a strong dynamical constraint, it can be used to track the evolution of fluid parcels. One can find PV mathematically expressed in various ways, but here we simply calculate potential vorticity as the ratio of absolute vorticity and a scale height, expressed here through the vertical density gradient:

$$
q=\frac{\zeta_{\rho}+f}{h}=\frac{\left(\zeta_{\rho}+f\right)}{\rho_{0}}\left|\frac{\partial \rho}{\partial z}\right|=\frac{\left(\zeta_{\rho}+f\right)}{\rho_{0}}\left|-\alpha \rho_{0} \frac{\partial T}{\partial z}\right|=\alpha\left(\zeta_{\rho}+f\right)\left|\frac{\partial T}{\partial z}\right|,
$$

where $\zeta_{\rho}=\left(\partial_{x} v-\partial_{y} u\right)_{\rho}$ is the relative vorticity taken along local surfaces of constant density and $f=2 \Omega \sin (\phi)$ is the planetary vorticity. We have used the linear, salt-free equation of state $\rho=\rho_{0}\left[1-\alpha\left(T-T_{0}\right)\right]$ to relate density and temperature, where $\alpha$ is the coefficient of thermal expansion, $\rho_{0}$ is a reference density, and $T_{0}$ is a reference temperature. The specific values of the reference quantities are irrelevant since they drop out of our calculation.

The large-scale PV distribution and its meridional gradient along surfaces of constant density are shown in Figure 4.9. The field is dominated by the planetary vorticity and stratification; the relative vorticity is typically an order of magnitude smaller than the planetary component. Increased wind stress changes the large-scale PV field by altering the buoyancy structure, particularly near the surface throughout the gyre region. A sharp PV gradient is found across the gyre-channel transition latitude, and stronger wind stress increases the steepness of this gradient by about 55\%. The PV gradient reverses sign in the gyre region, indicating a likely area of instability. The sensitivity of the density structure to changing wind stress is not surprising, since our model does not explicitly resolve mesoscale eddies. Despite this limitation, it is useful to compare results from eddy-parameterized and eddy-permitting models to better understand the strengths and weaknesses of current parameterization schemes. 

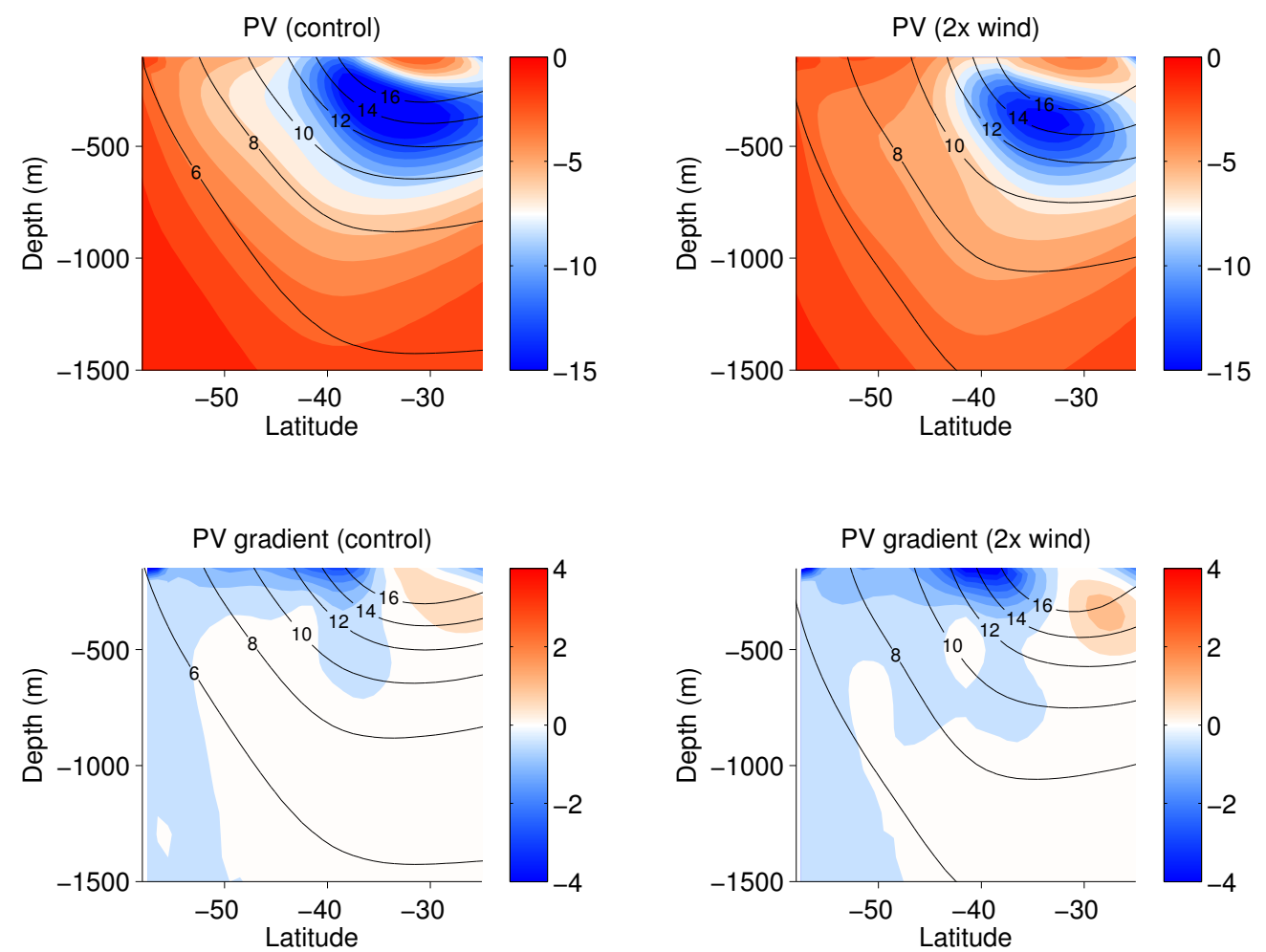

FIG. 4.9. Approximate equilibrium zonal mean potential vorticity (top row, units of $10^{-11}$ $1 / \mathrm{ms}$ ) and its meridional gradient along surfaces of constant temperature (bottom row, units of $10^{-16} 1 / \mathrm{m}^{2} \mathrm{~s}$ ). Shown are the control (left column) and doubled wind (right column) cases, with zonal mean temperature $\left({ }^{\circ} \mathrm{C}\right)$ in solid black contours. 


\subsection{TRANSIENT RESPONSE}

The transient response of the interior ocean to large-scale changes in atmospheric forcing is typically carried out by Rossby waves (Chelton and Schlax, 1996). Rossby waves can be described by an infinite number of vertical normal modes in order of decreasing phase speed. The lowest order (barotropic) mode is uniform with depth and can cross an ocean basin in about a week. The next higher order (first baroclinic) mode changes sign with depth and takes several months to decades to cross an ocean basin. In our model domain, the phase speed (and group velocity) of the first baroclinic mode is dominated by the mean advection, such that it can cross the model domain at channel latitudes in 2-3 years.

In this section, we discuss the ocean's approach to equilibrium following a step function change in Southern Hemispheric wind stress. We examine the adjustment of the largescale buoyancy structure, the pycnocline depth, isopycnal slope, zonal mean velocity, and northward heat transport. Overall, we find two distinct areas of subsurface warming. The high-latitude warming results from the increased amplitude of the standing-wave pattern of the circumpolar current, i.e. from meandering, and the low-latitude warming is induced by

the spin-up of the subtropical gyre and the associated increase in Ekman pumping. First, we examine the time-dependent adjustment of the zonal mean buoyancy structure in order to visualize and quantify these two warming signatures.

\subsubsection{Buoyancy structure}

In Figure 4.10 we plot the anomalous (doubled wind minus control) zonal mean buoyancy as a function of latitude and depth. During the first few weeks and months after the change in forcing, a cold region develops in the channel due to enhanced equatorward Ekman transport of high-latitude cold water at the surface. The cold anomaly penetrates into the interior following Eulerian mean streamfunction contours. Over the next ten years, the cold surface 

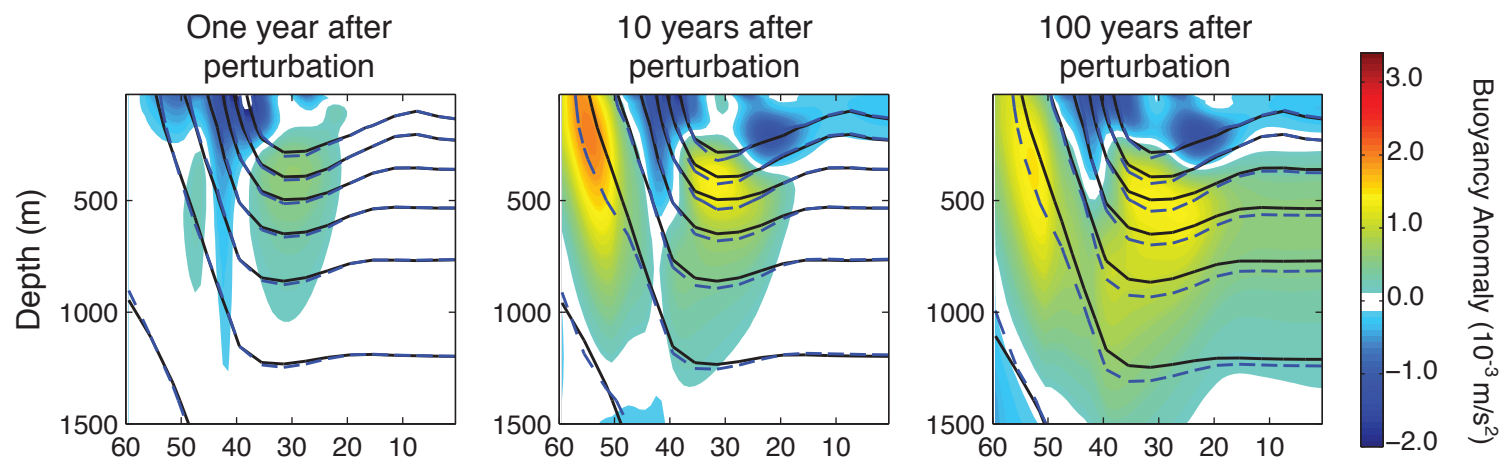

Fig. 4.10. Zonal mean anomalous (doubled wind minus control case) buoyancy structure as a function of latitude and depth at one (left), ten (middle), and 100 years (right) after the step function change is applied. The lines are zonal mean isotherms (every $2{ }^{\circ} \mathrm{C}$ up to 16 ${ }^{\circ} \mathrm{C}$ ) from the control (solid) and doubled wind (dashed) experiments.

anomaly persists, and regions of subsurface warming develop at high latitudes and across the latitude band of the subtropical gyre. The high-latitude warming is likely caused by increased poleward heat transport via the stationary wave component, and the midlatitude warming reflects stronger Ekman pumping associated with the spin up of the subtropical gyre. The time series of both the high-latitude and gyre latitude warming signatures suggests that they are indeed brought about by different mechanisms (see Figure 4.11). The highlatitude warming takes a little over a decade to fully develop, which is consistent with the timescale of the poleward shift in the maximum extent of the circumpolar current contours (see Figure 4.12) and with the propagation speed of low-order baroclinic Rossby waves. The low-latitude warming is weaker and more gradual, increasing in a linear fashion over several decades as the subtropical gyre spins up. Surfaces of constant buoyancy are deepened by both the low-latitude and the high-latitude warming, which by year 100 penetrates through the tropics. The isopycnal tilt does change locally, but there is no obvious large-scale steepening of buoyancy surfaces. The steep change in slope seen in Figure 4.4 takes many centuries to fully develop as the global pycnocline adjusts. 

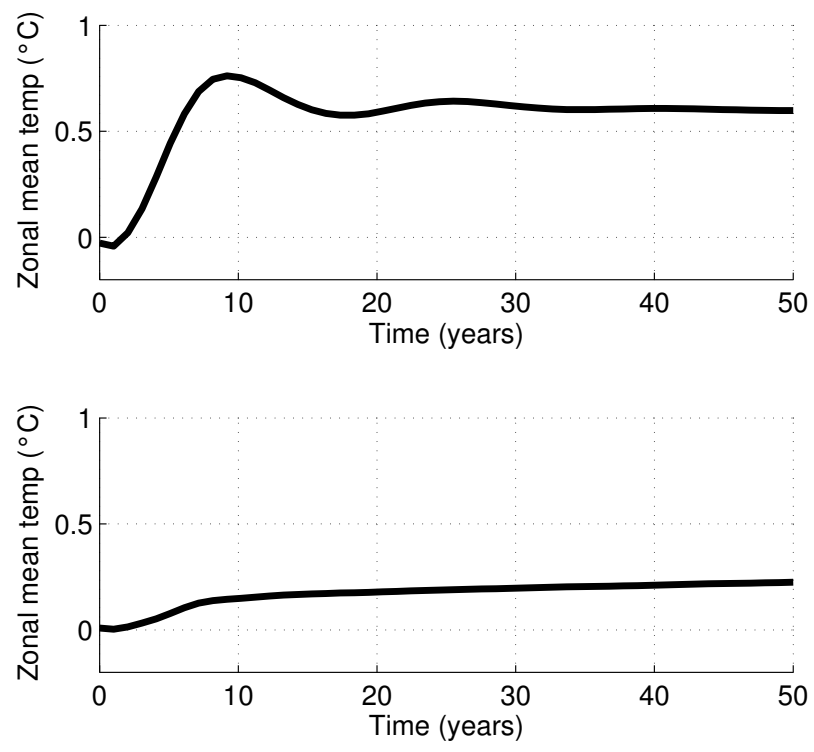

Fig. 4.11. Average temperature anomaly at high latitudes $\left(60-50{ }^{\circ} \mathrm{S}\right.$, top $)$ and gyre latitudes (40-30 ${ }^{\circ} \mathrm{S}$, bottom).

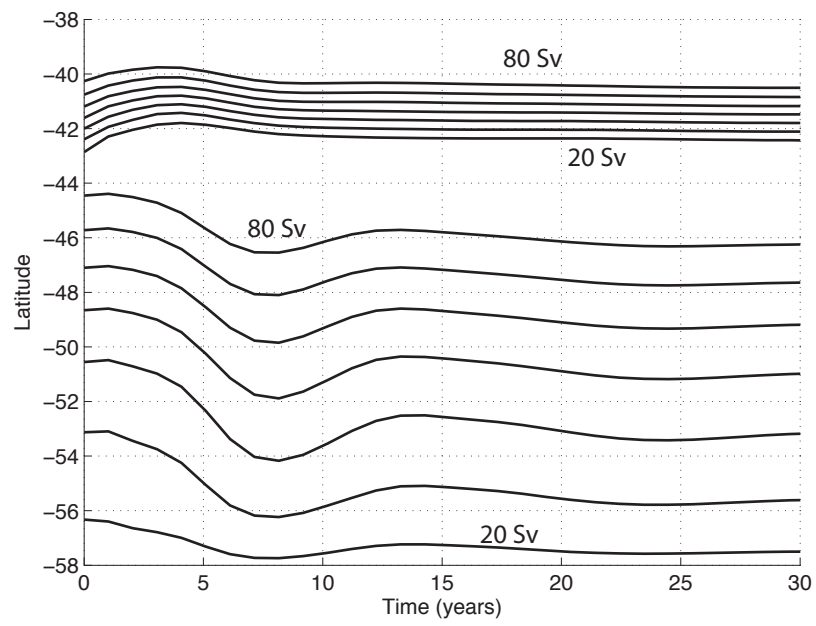

FIG. 4.12. Maximum and minimum latitudes of several barotropic streamfunction contours from the doubled wind run. Contours are from $20 \mathrm{~Sv}$ to $80 \mathrm{~Sv}$ in steps of $10 \mathrm{~Sv}$. The upper lines show the maximum northward extents and the lower lines illustrate the maximum southward extents of the contours. 


\subsubsection{Interannual adjustment}

It is difficult to infer the behavior of the slope field from Figure 4.10. To get a better sense of the behavior of the slope field in the core of the jet, we plot a Hovmöller-type diagram (Hovmöller, 1949) of slope as a function of depth and time (Figure 4.13). During the first year, surface isopycnals tilt up in response to increased northward transport in the wind-driven Ekman layer. Around year four, the slope flattens back out at all depths for a year or two. After this somewhat sudden flattening event, isopycnals steepen monotonically until settling into an equilibrium configuration. It is clear that the control model is not completely in equilibrium, since the slope continues to evolve on a multi-century timescale. As such, we have to keep in mind that both the adjustment of the doubled wind case and the slow drift of the control model may show up in the anomalous (doubled wind minus control) fields. Note that in the doubled wind case, the slope field appears approximately static after 500 years. Stronger wind stress appears to have somewhat suppressed the long-term adjustment that is evident in the control model.

The slope flattening event is contemporary with a burst of anomalously strong poleward heat transport. To better understand this feature, we examine the heat transport immediately after the profile switch and onwards. Within a few rotation periods after the wind is strengthened, wind-driven Ekman transport increases northward heat transport in the equatorward flank of the channel, dominating net transport for the first several months over the latitude band where maximum wind stress is applied. The surface stationary eddy-induced circulation responds within a few months, and the full depth-integrated stationary eddydriven poleward transport gets stronger as deeper isopycnal layers are tilted up. Ekman transport dominates the net transport for most of the first year, but by the end of the first year eddy driven transport dominates the net transport in the channel. The gyre spins up and the poleward heat transport associated with the gyre intensifies. Notice that the gyre strength and the channel eddy strength seems to "overshoot" and then oscillate about new 

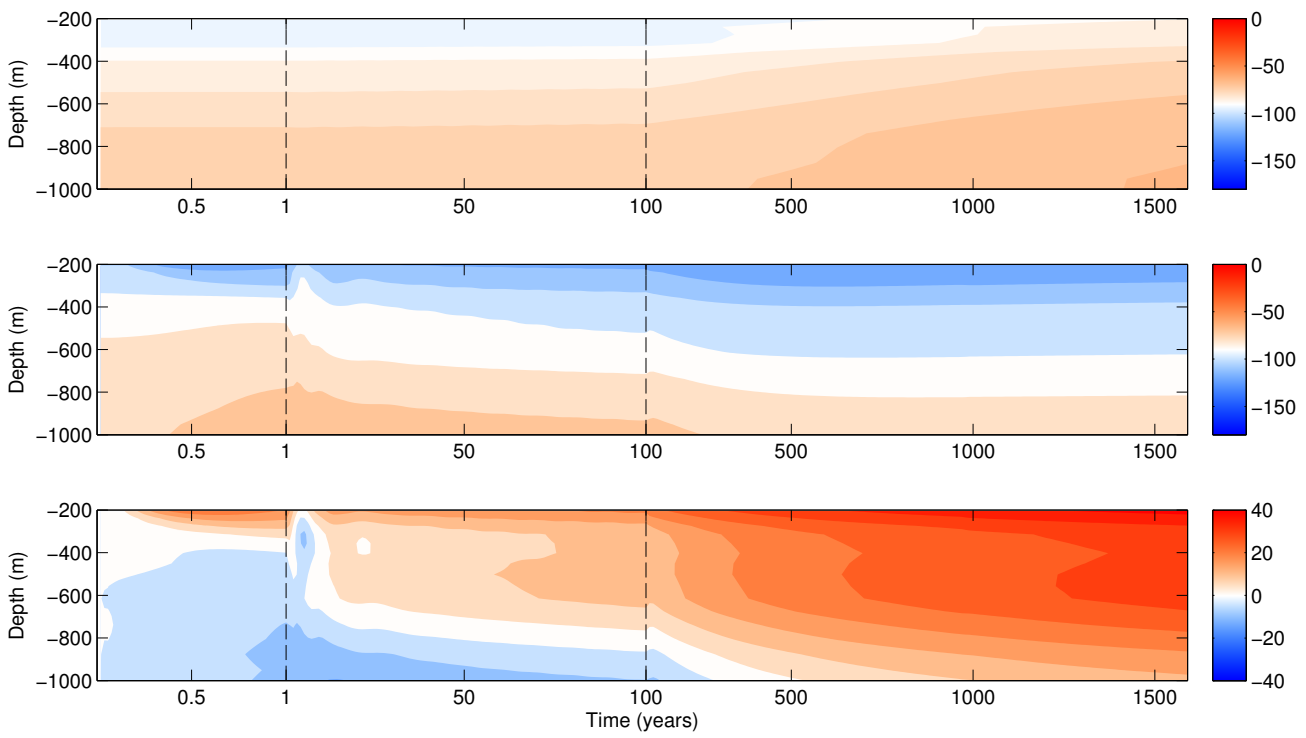

FIG. 4.13. Hovmöller diagram of control (top), doubled wind (middle), and anomalous slope (bottom, \% difference) using different time resolutions. The (dimensionless) zonal mean slope is defined by $\overline{s_{\rho}}=-M^{2} / N^{2}$ at each grid point. The dashed line at one year marks the transition from weekly to yearly averages, and the dashed line at 100 years marks the transition from yearly to decadal averages. Average over latitude is from $45{ }^{\circ} \mathrm{S}$ to $40{ }^{\circ} \mathrm{S}$, which contains the core of the zonal mean jet.

equilibrium positions with a multiple-year timescale. This seems to happen just before the maximum latitudes bend southward (Figure 4.12) and right around the same time as the slope flattening event. The slope overshot, flattened during a release of energy, and tilted back up to its new equilibrium. This appears to be a stationary wave dominated response consistent with a long Rossby wave propagating around the circumpolar channel.

\subsubsection{Decadal-scale adjustment}

In Chapter 2, we predicted that the pycnocline depth adjusts on a multi-decadal timescale. In order to test this hypothesis in the numerical model, we must diagnose the pycnocline depth using the global time-mean buoyancy distribution:

$$
D(x, y)=\frac{\int_{-H}^{0} z \bar{b}(x, y, z) d z}{\int_{-H}^{0} \bar{b}(x, y, z) d z}
$$



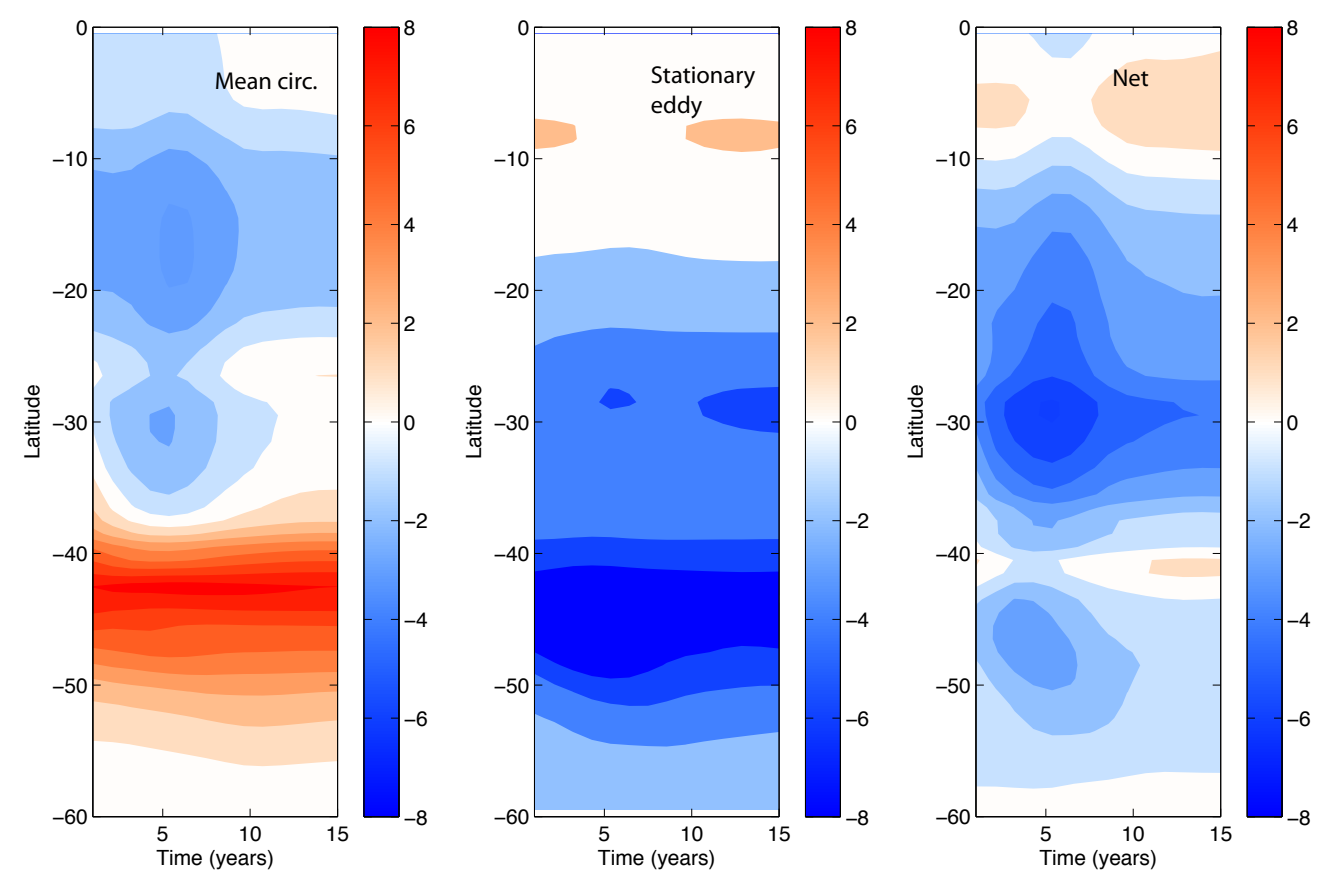

FIG. 4.14. Hovmöller diagram of mean northward heat transport $\left(10^{7} \mathrm{mK} / \mathrm{s}\right)$ from the mean circulation (left), stationary eddy circulation (middle), and their sum (right). The heat flux due to transient eddies is an order of magnitude smaller than these fields and is not shown.

where $H$ is the depth of the bottom topography and the bar represent a time average. The mean pycnocline depth is determined by taking the spatial average of equation 4.9,

$$
[D]=\frac{1}{A} \oint_{\Sigma} D(x, y) d A,
$$

where $\Sigma$ denotes the surface area $A$ over which the integration is performed. Averages are taken over all longitudes and in selected latitude bands. In Figure 4.15, we plot the results of taking averages over the southernmost, middle, and northernmost thirds of the domain, as well as over the full area of the basin. We see that the southernmost pycnocline deepens more than the other basins, as expected. The northern third does not show any significant change for the first century or so, indicating the long timescale of interhemispheric adjustment. Both the control model and the doubled wind model continue adjusting for several millennia after the profile switch (Figure 4.16). After 1000 years or so in the doubled wind simulation, we see continued adjustment that is likely from the slow lower-cell equilibration still ongoing in both the control and doubled-wind model. Our conclusions are not negatively affected by the 
continued adjustment of the control model, but it should be kept in mind that the control model is not itself in thermodynamic equilibrium. The anomalous (doubled wind minus control) depth serves as a reasonable indicator of the circulation and buoyancy changes forced by the intensified wind field.

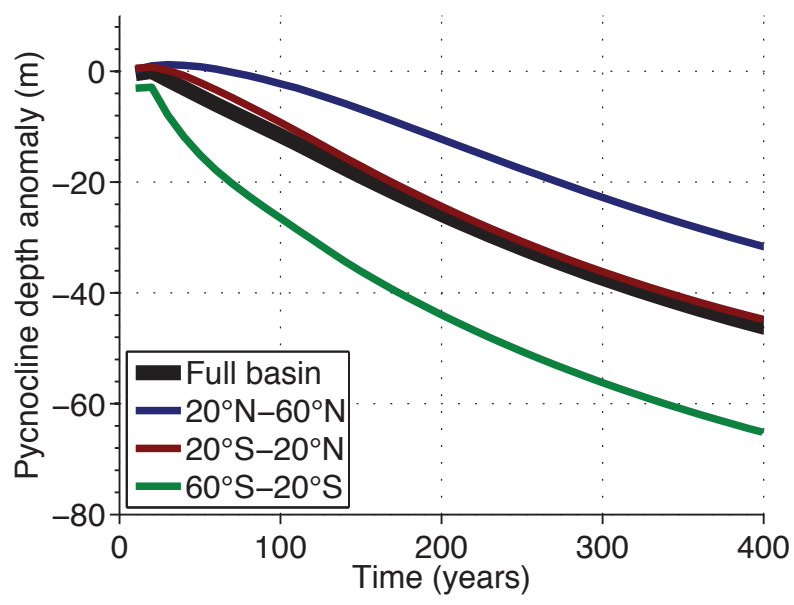

Fig. 4.15. Anomalous (doubled wind minus control) pycnocline depth by region. Figure adapted from Jones et al. (2011).

Though a direct comparison of equilibration timescale is difficult, the conceptual model presented in Chapter 2 predicts a much shorter e-folding timescale (20-25 years when using geometric parameters appropriate for the sector model) than the adjustment timescales seen in numerical integrations using the sector model (the e-folding timescale of the anomalous pycnocline depth is around 560 years in the coarse resolution model). The conceptual model may not be capable of capturing the longest, centennial-order response timescales associated with the adjustment of subtropical gyre circulation and Antarctic Bottom Water circulation. The simple nature of the theoretical scalings might also explain the difference in timescale between the conceptual and numerical models. Despite this limitation, the conceptual model is still a useful guide for understanding multi-decadal adjustment.

We may also wish to diagnose the volume transport of the circumpolar current across a 

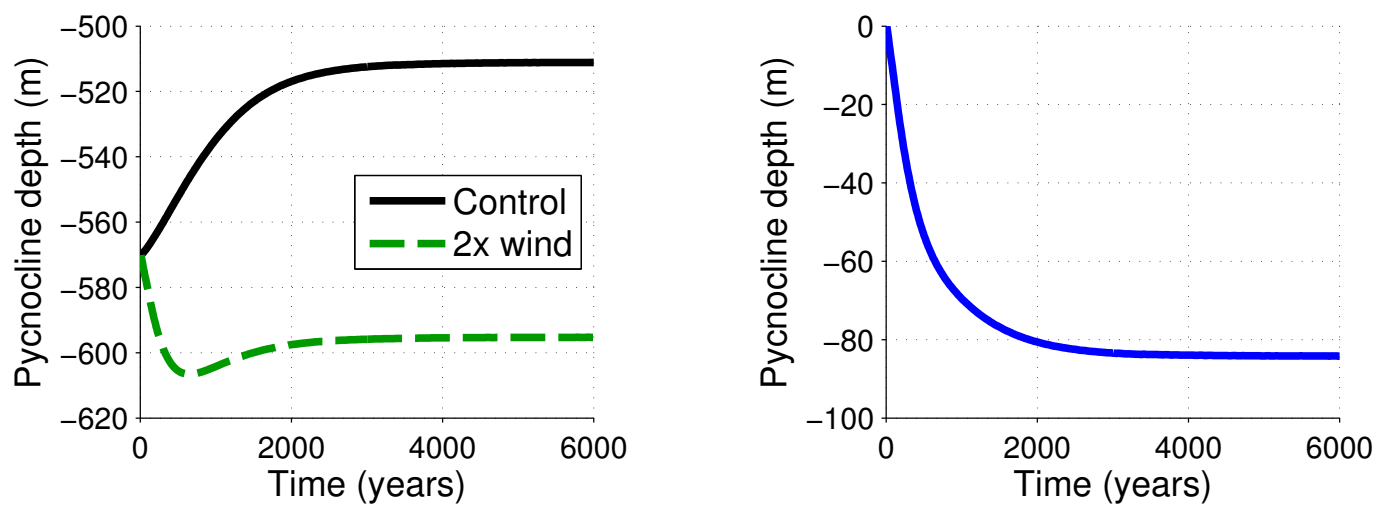

FIG. 4.16. Global mean pycnocline response following the step function change in wind stress profile. Both the control and doubled wind values are plotted in the left panel, and the anomalous value (doubled wind minus control) is shown in the right panel.
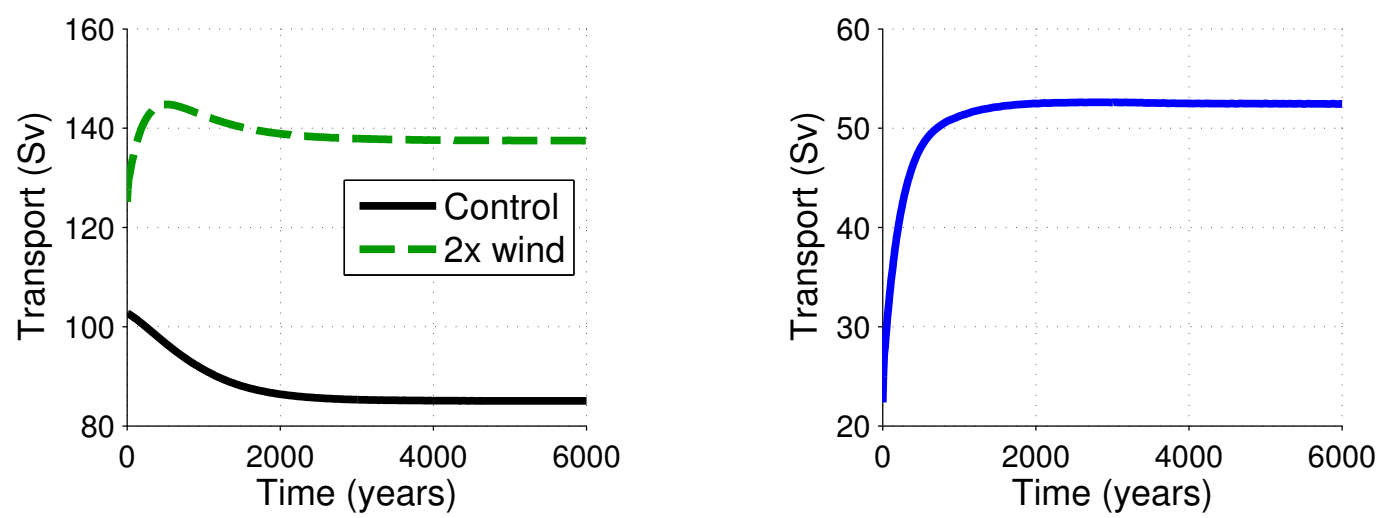

FIG. 4.17. Volume transport ( $\left.1 \mathrm{~Sv}=10^{6} \mathrm{~m}^{3} / \mathrm{s}\right)$ through the channel opening following the step function change in wind stress profile.

single meridional cut through the channel:

$$
T_{C C}=\int_{-H}^{0} \int_{y_{S}}^{y_{N}} u(x, y, z) d y d z
$$

where $y_{S}$ and $y_{N}$ are the southern and northern latitudes of the channel opening, respectively. The results are shown in Figure 4.17. Within one year, the transport jumps from 100 to 120 Sv. Over the next 10 years it increases to $130 \mathrm{~Sv}$, getting up to 140 within 300 years. The e-folding timescale of the anomalous transport is around 280 years, reflecting the centennial scale adjustment of Southern Ocean circulation to changes in wind stress, and the millennialscale adjustment is likely related to the formation of bottom water at high southern latitudes. 
As expected, eddy saturation is not observed in our coarse resolution model; much of the extra kinetic energy from stronger winds ends up in the mean flow.

\subsubsection{Double-gyre oscillation}

The bathymetry and imposed wind stress profile set up a double-gyre configuration in the Northern Hemisphere. The two-gyre system exhibits low-frequency (interannual) variability as seen in Hovmöller diagrams of sea surface height (Figure 4.18). The source of the variability is likely an unstable mode of steady state flow excited by interactions with the idealized bottom topography (Nauw and Dijkstra, 2001). The double-gyre oscillation is seen in both the control and the doubled wind numerical runs, though they appear to shift out of phase with one another following the change in wind stress profile.
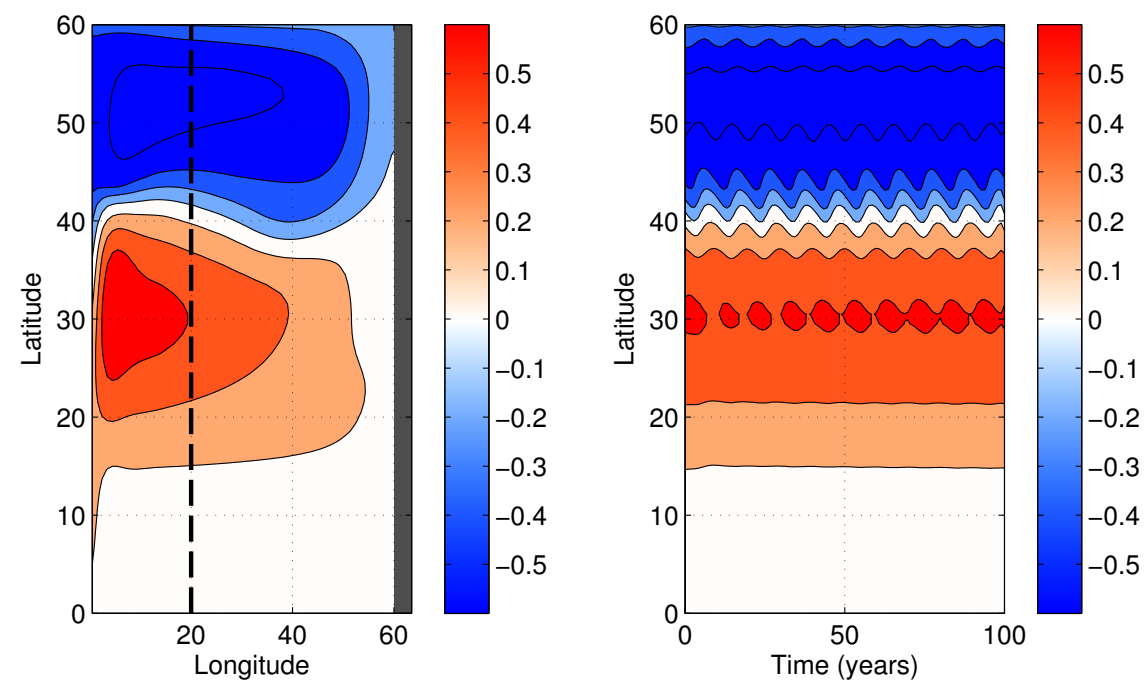

FIG. 4.18. Northern hemispheric gyre oscillation. (Left) annual mean SSH (m) and (right) Hovmöller of annual mean SSH at $20^{\circ} \mathrm{E}$ (the longitude of the dark dashed line in the lefthand figure). 


\subsection{WEAK WIND AND MERIDIONAL RIDGE}

In this section we present results from several additional coarse resolution experiments. First, we examine a "weak wind" scenario in which the maximum wind stress is instantaneously halved by switching from the control profile to a profile that is weaker in the Southern Hemisphere. The reduced wind profile is still sinusoidal, but the maximum wind stress in the Southern Ocean is cut down to $0.065 \mathrm{~Pa}$. We find that the average circumpolar current slows down by about $22 \%$ and the current profile becomes more zonal (Figure 4.19). Circumpolar transport decreases to an equilibrium value of around $80 \mathrm{~Sv}$. The half-wind transport adjusts to the change in wind stress somewhat faster than that of the doubled wind case, which is consistent with the prediction from Chapter 2 that the equilibration timescale is relatively short in the weak-wind regime. We also examine the zero-wind case, wherein the momentum forcing is turned off entirely while the buoyancy forcing is held constant. After the wind stress is switched off entirely, the circumpolar current spins down over the course of several centuries, though it does not disappear entirely (see Figure 4.20). The meridional temperature gradient supported by buoyancy forcing is indeed capable of driving a relatively slow, broad circumpolar current with weak vertical shear.

In order to better understand the effect of bathymetry on the spatial pattern of the circumpolar current, we conduct an additional step response experiment constrained by the addition of a meridionally-oriented mid-ocean seamount (Figure 4.21). Frictionless flow must still follow contours of $f / h$, which are typically more zonal due to the addition of the ridge. As such, the bathymetry is expected to suppress the stationary wave response by partially "locking in" the position of the current via topographic steering. Interestingly, under control wind-forcing conditions the circumpolar transport is somewhat higher in the seamount case. Under doubled-wind conditions, the circumpolar current is indeed faster than in the control

case; the increase is about the same as that seen in the step response experiment without a mid-ocean ridge. However, in the doubled wind case the transport drops rapidly after the 

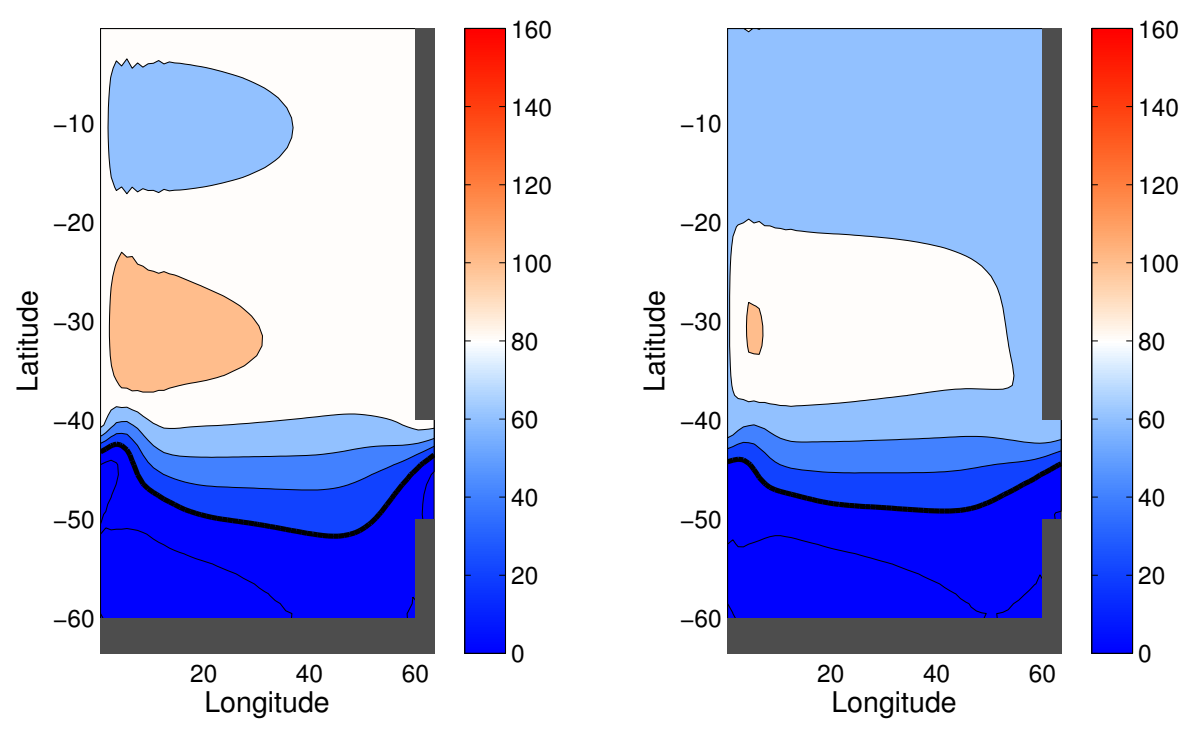

FIG. 4.19. Control (left) and doubled wind (right) simulation barotropic streamfunctions (Sv) for the half-wind experiment. $20 \mathrm{~Sv}$ contour is darkened, and the contour interval is 20 Sv.

period of initial adjustment. In the strong-wind regime, the seamount provides an additional source of topographic form stress that extracts momentum from the flow over sufficiently long timescales. It may be that this effect is reduced in the relatively weak-wind (control) case. We further discuss the seamount constraint using eddy-permitting configuration in a wind-driven experiment in section 6.2.

\subsection{DISCUSSION}

While the step-response approach is not particularly realistic for studying decadal-scale changes in forcing, it is a useful method for investigating the ocean's adjustment to a new equilibrium state following a significant perturbation. Here we summarize the results of our coarse-resolution step response experiment and discuss the implications for the basic theories outlined in Chapters 1 and 2 . 


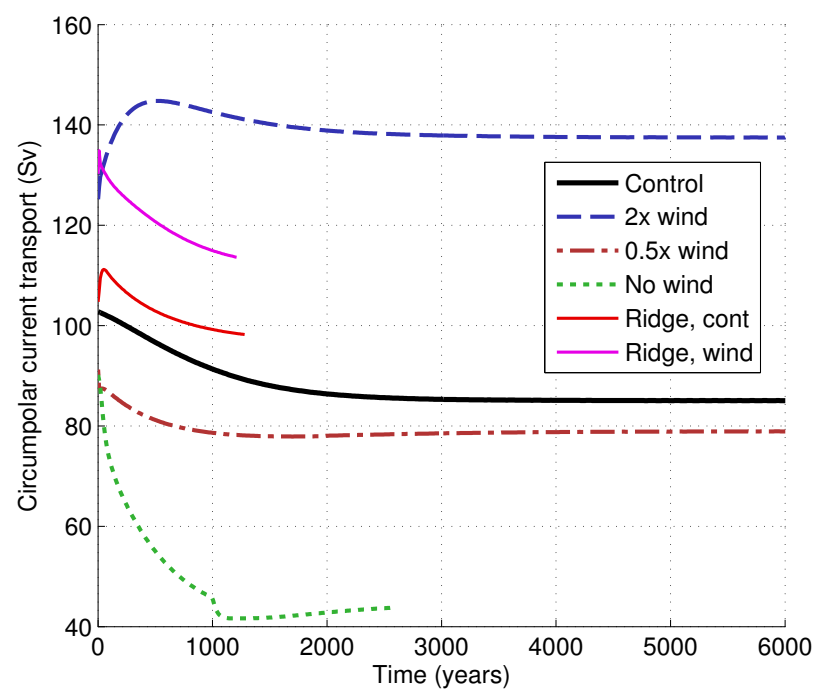

FIG. 4.20. Circumpolar current transport $\left(1 \mathrm{~Sv}=10^{6} \mathrm{~m}^{3} / \mathrm{s}\right)$ time series using six different surface wind profiles, including the control (thick black), doubled wind (blue dashed), halfwind (red dash-dot), and zero-wind (green dash) profiles. Also shown is the transport from runs with an additional meridional ridge (thin solid lines).
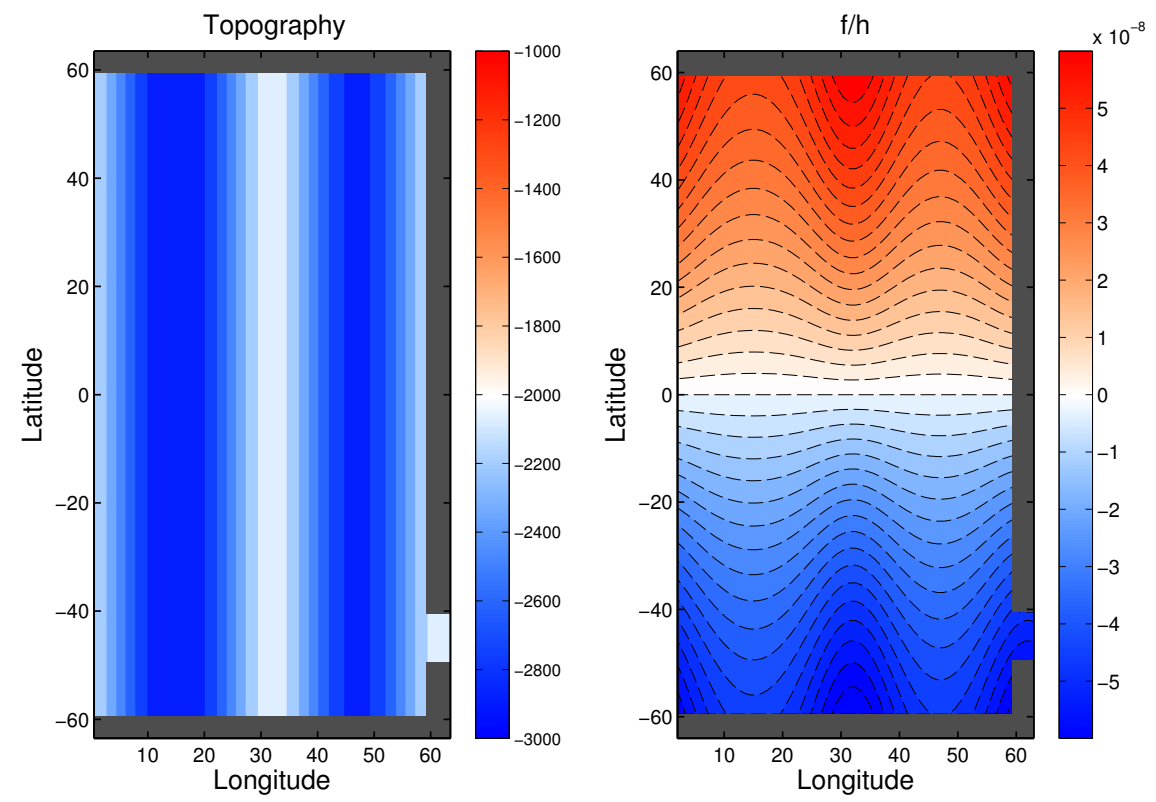

FIG. 4.21. Bathymetry used in the mid-ocean seamount step response experiment (left) and associated contours of $f / h$ (right), where $h$ is taken to be the entire depth of the ocean. 
Within a few rotation periods following the increase in wind stress, enhanced equatorward Ekman transport creates a cold anomaly in the channel near the surface, strengthens subpolar upwelling, and intensifies subtropical Ekman pumping. The barotropic contours of the circumpolar current broaden over the course of the first decade, in the sense that the current deviates further northward downstream of the channel and further southward across the basin interior. The result is warming in the subpolar region brought about by enhanced meandering of the stationary pattern of the circumpolar current. Over the course of the next several decades, the subtropical gyre continues to spin up and warm the interior. These two warming patterns deepen the Southern Ocean isotherms without significantly changing their slope for the first century or so. It takes many decades to centuries for the wind-induced changes in stratification in the Southern Ocean to communicate with northern basins through the adjustment of the global pycnocline. Isopycnal slope gradually changes as the global pycnocline deepens. The behavior of the coarse resolution primitive equation model is qualitatively consistent with the predictions discussed in Chapter 2.

It is widely recognized that coarse resolution models do not correctly represent the response of the eddy field to changes in wind stress, particularly in the Southern Ocean. Any conclusions we attempt to draw regarding eddy compensation and saturation would be premature. That being said, isopycnal slope and the residual circulation do indeed change in this experiment, lending some support to the idea that departures from eddy compensation may be relevant to centennial-scale adjustment. In the next chapter, we re-run the step response experiment using eddy-permitting horizontal resolution in order to gain a more realistic representation of mesoscale variability. 


\section{EDDY PERMITTING STEP RESPONSE EXPERIMENTS}

In Chapter 4, we performed a step response experiment by instantaneously switching from a weaker wind stress profile to a stronger one and tracking the adjustment process. In this chapter, we repeat our experiment using a finer horizontal grid $\left(1 / 6^{\circ} \times 1 / 6^{\circ}\right)$ capable of resolving mesoscale eddies. We call it an eddy-permitting configuration instead of an eddyresolving one because it does not resolve eddies down to the smallest scale; it only permits the existence of larger mesoscale features. Sub-grid scale eddy fluxes are still parameterized. The eddy permitting model exhibits a great deal more spatial and temporal variability compared to the coarse resolution model, much of it related to fluid dynamical instabilities and the formation, propagation, and dissipation of Rossby waves. The density field displays evidence of decadal and centennial adjustment to a change in surface wind stress that is roughly consistent with (though perhaps somewhat weaker than) the long-term equilibration process seen in the coarse resolution model.

As in Chapter 4, we begin by examining the change in momentum and buoyancy fluxes at the surface in section 5.1. Since the eddy-permitting variant of our model is still computationally expensive to run, we were unable to run a step response experiment to full thermodynamic equilibrium. Nevertheless, we can learn something about the decadal-to-centennial scale adjustment process by examining differences between the control and doubled wind

runs. We examine the quasi-equilibrium state in section 5.2. The transient adjustment of our eddy permitting model is discussed in section 5.3. Finally, we explore the nature of mesoscale interannual variability in the gyre and channel region of the model in section 5.4.

\subsection{SURFACE FLUXES}

As discussed in section 4.1, air-sea heat flux is parameterized using a Newtonian relaxation scheme $H=-\lambda_{T}\left(T-T_{s}\right)$, where $T_{s}=T_{s}(y)$ is the prescribed surface temperature 
profile and $1 / \lambda_{T}$ is a restoring timescale (set to about one month). The annual mean net air-sea heat flux displays fine spatial structure as permitted by improved horizontal resolution (Figure 5.1). The ocean absorbs heat from the atmosphere in the tropics (primarily on the western side of the basin) and releases heat to the atmosphere at middle and high latitudes. A region of strong heat uptake exists on the eastern side of the basin. The overall midlatitude pattern of air-sea heat flux is indicative of the subtropical gyre that transports warm water southward and cold water northward. Under enhanced wind conditions (i.e. when the momentum is forced by the doubled-wind profile), heat uptake is enhanced in the eastern subtropics and in the western subtropics. Overall, enhanced wind stress causes the midlatitude ocean to take up more heat (or at least cool more slowly) from the atmosphere compared to the control case, particularly in the latitude band of the gyre and channel.
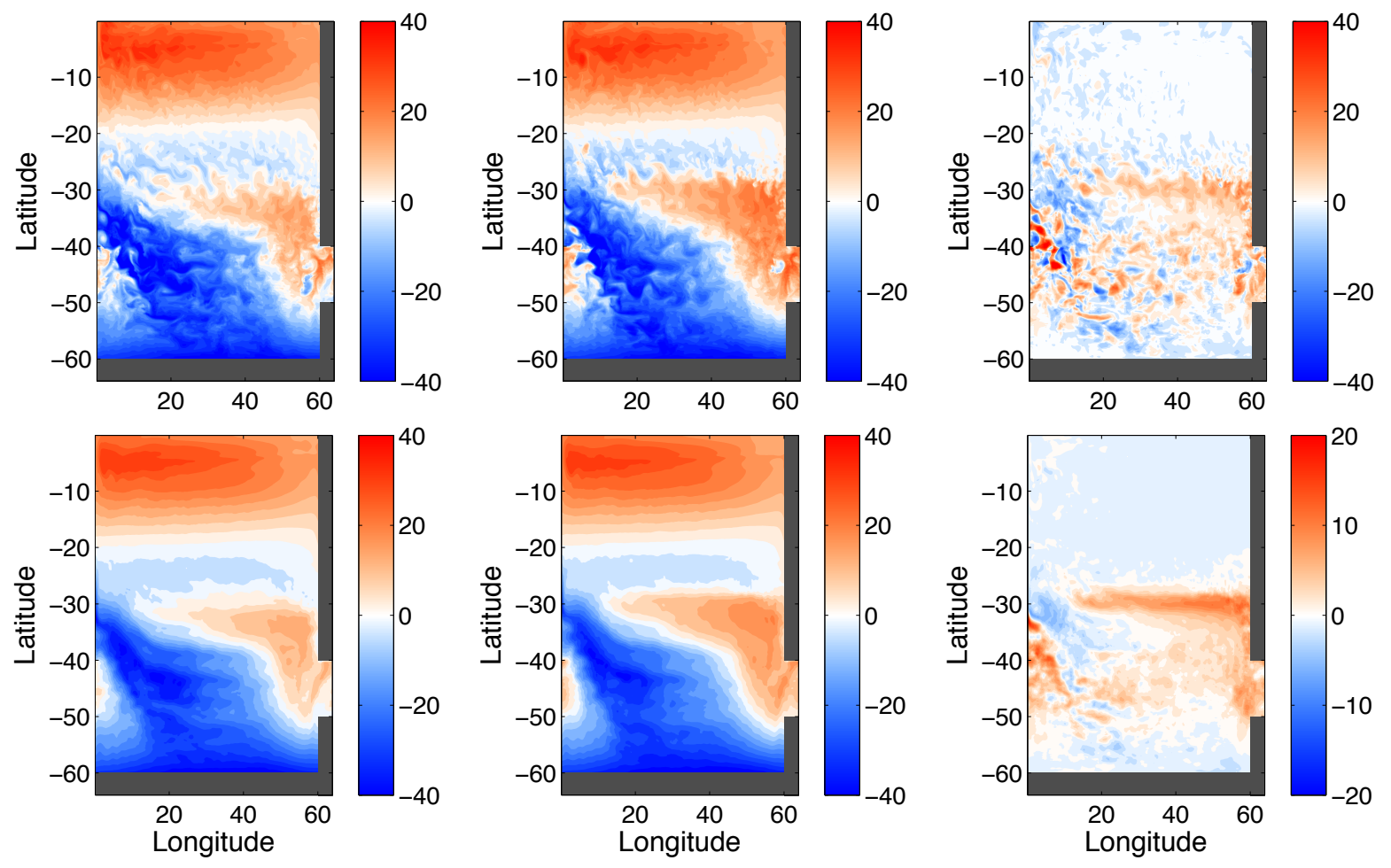

FIG. 5.1. Five-day average (top row) and annnual mean (bottom row) net air-sea heat flux $\left(\mathrm{W} / \mathrm{m}^{2}\right)$ for the control (left) and doubled wind (right) simulations. The anomaly (doubled wind minus control) is shown on the right. Positive values indicate heat flux into the ocean. 
Next we calculate the power input by the wind into the general circulation, i.e. $P=$ $\left\langle\tau \cdot \mathbf{v}_{\mathbf{g}}\right\rangle$ as discussed in section 4.1.2. The pattern (shown in Figure 5.2) is quite different from that seen in the coarse resolution case (Figure 4.2). Instead of a strip of positive power input that stretches across the channel, we find several narrow bands of alternating sign. Since the wind stress is positive everywhere south of $30^{\circ} \mathrm{S}$, the sign of the power input indicates the direction of the zonal component of the current, and we can conclude that both the gyre and channel regions of the domain host narrow, alternating jets. Some topographic steering is evident on the western side of the jets where the current deviates to the north. The banded pattern of shown in Figure 5.2 does not completely vanish under decadal averaging (the averaging period used in the coarse resolution case), but it does get weaker. The pattern is unique to the fine-resolution model; it is generated by mesoscale eddies and the variability associated with them. We discuss the dynamics of the alternating jets further in section 5.4.
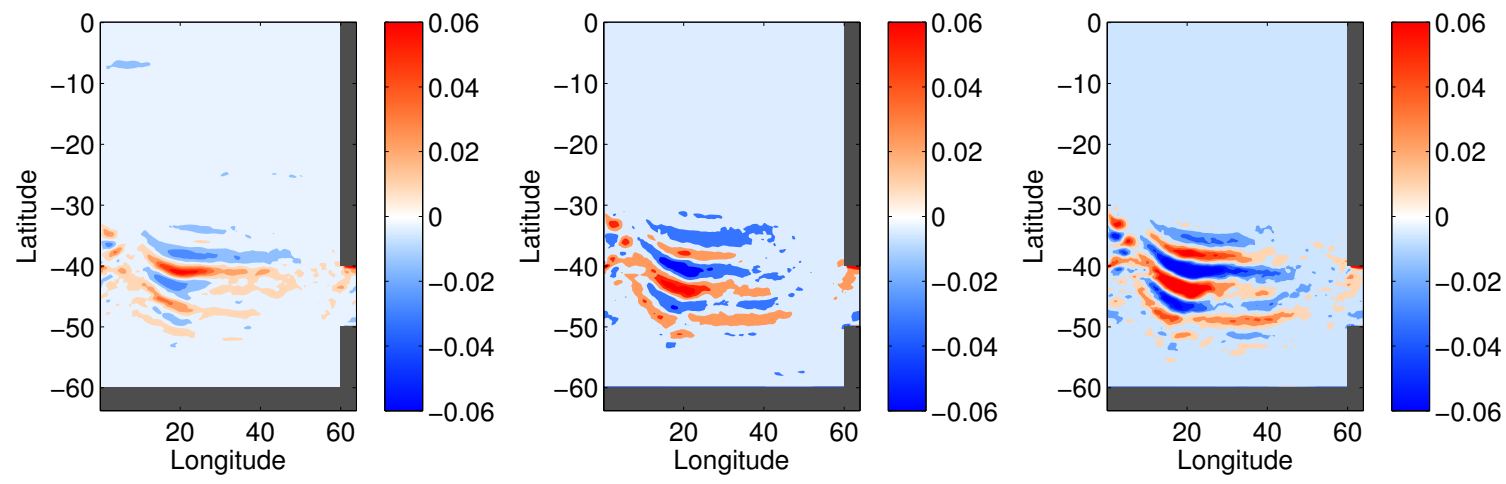

FIG. 5.2. Annual mean power input $\left(\mathrm{W} / \mathrm{m}^{2}\right)$ from the wind to the general circulation about 400 years after the wind is doubled. Shown are the control experiment (left), the doubled wind experiment (middle), and the doubled wind minus control case values (right).

\subsection{QUASI-EQUILIBRIUM}

In this section, we examine some of the differences between the control and doubled wind experiments in an effort to better understand the decadal to centennial scale adjustment of the ocean to changes in surface wind stress. We were unable to run the model 
to full thermodynamic equilibrium due to computational constraints, so our results are not definitive. Despite this limitation, our numerical experiment still provides insight into the decadal scale response of the buoyancy and eddy fields to changing wind stress. Oceanic stratification is at least weakly sensitive to wind stress on these longer timescales.

\subsubsection{Sea surface height}

First we examine the change in decadal mean sea surface height between the control and doubled wind runs. Much of the difference between the two runs (i.e. the "adjustment signal") is dominated by the presence of mesoscale variability (Figure 5.3, right panel). The bands of alternating rising/falling SSH represent the decadal-scale shift in the position of "tracks" of eddy activity along which the anomalies (i.e. oceanic "weather") preferentially propagate. The "storm tracks" shift on a decadal scale in both the control and the doubled wind runs, and the anomalies themselves propagate along the tracks on interannual and shorter timescales, as discussed further in section 5.4. It is difficult to detect the spin-up of the subtropical gyre and circumpolar current beneath the mesoscale noise, especially given the relatively short record available for the eddy permitting model. We must examine a few other metrics in order to quantify the gyre and circumpolar current response.
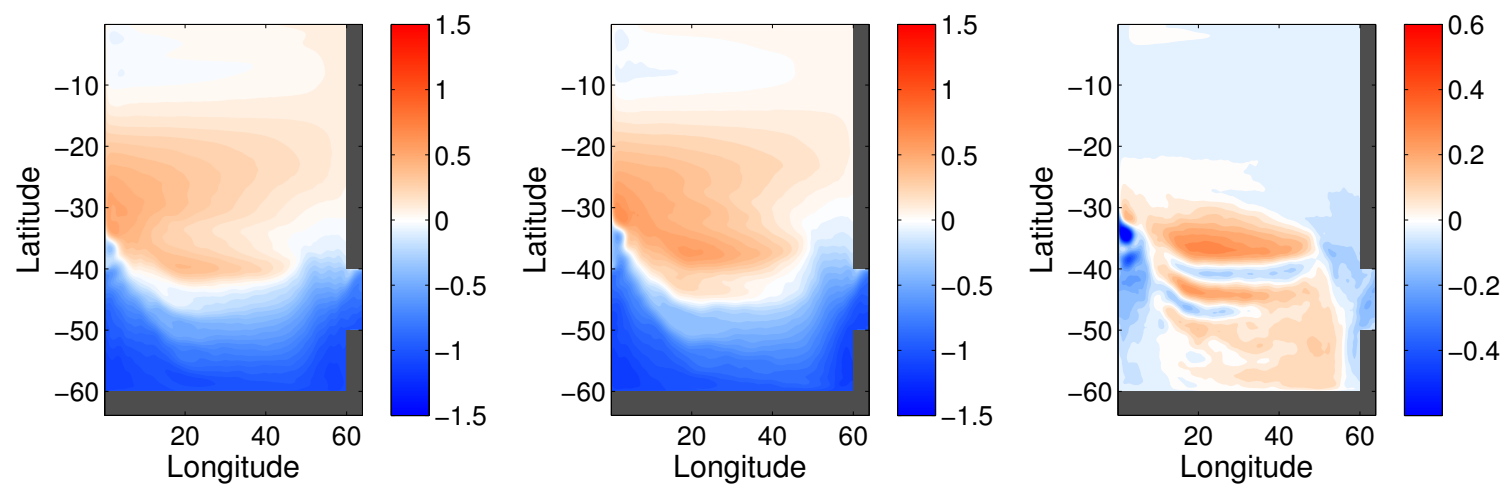

FIG. 5.3. Decadal mean sea surface height $(\mathrm{m})$ for the control (left) and doubled wind (middle) cases from years 90-100 in the step response experiment. The anomaly (doubled wind minus control) is shown in the right panel. 


\subsubsection{Zonal mean zonal velocity}

In Figure 5.4 we plot the depth-integrated zonal mean zonal velocity for both the control (solid black lines) and doubled wind (green dashed lines) cases for two different time periods. In the first decade following the step change in wind stress, a strong jet forms in the channel, and its mean position shifts by roughly $3^{\circ}$ southward as the stationary wave pattern of the circumpolar current adjusts to stronger forcing, in a manner similar to that seen in the coarse resolution experiment (see section 4.3). The mean jet "overshoots" its equilibrium latitude (which appears to be around $42^{\circ} \mathrm{S}$, as derived from a Hovmöller diagram of zonal velocity with latitude) and oscillates about it on a multi-decadal timescale. An average performed over years 300-390 reveals a sharp jet that sits near its approximate equilibrium position. The jets also display a considerable amount of vertical coherence, in that the zonal mean velocity typically changes by less than $1 \%$ in the top $1000 \mathrm{~m}$ of the ocean. It should be noted that the mean jet position is essentially an oceanic "storm track" along which strong anomalies propagate, as opposed to a zonal mean current as seen in the coarse resolution case. Hovmöller diagrams reveal a significant amount of energy at the mesoscale in the eddy-permitting run.

\subsubsection{Depth-integrated circulation}

We evaluate the barotropic streamfunction as discussed in section 4.2.5. We see that the contours associated with the circumpolar current meander in the meridional direction across the basin (Figure 5.5), much like those in the coarse resolution case (Figure 4.7). We can describe this pattern as a stationary wave that is at least in part topographically induced and sensitive to wind forcing. Eddies generate considerable spatial and temporal variability along the length of the stationary wave. In the annual mean we see a collection of zonal jets of alternating sign, as seen in the momentum flux field (Figure 5.2). There is also a strong western boundary current (WBC) that interacts with the portion of the circumpolar current 

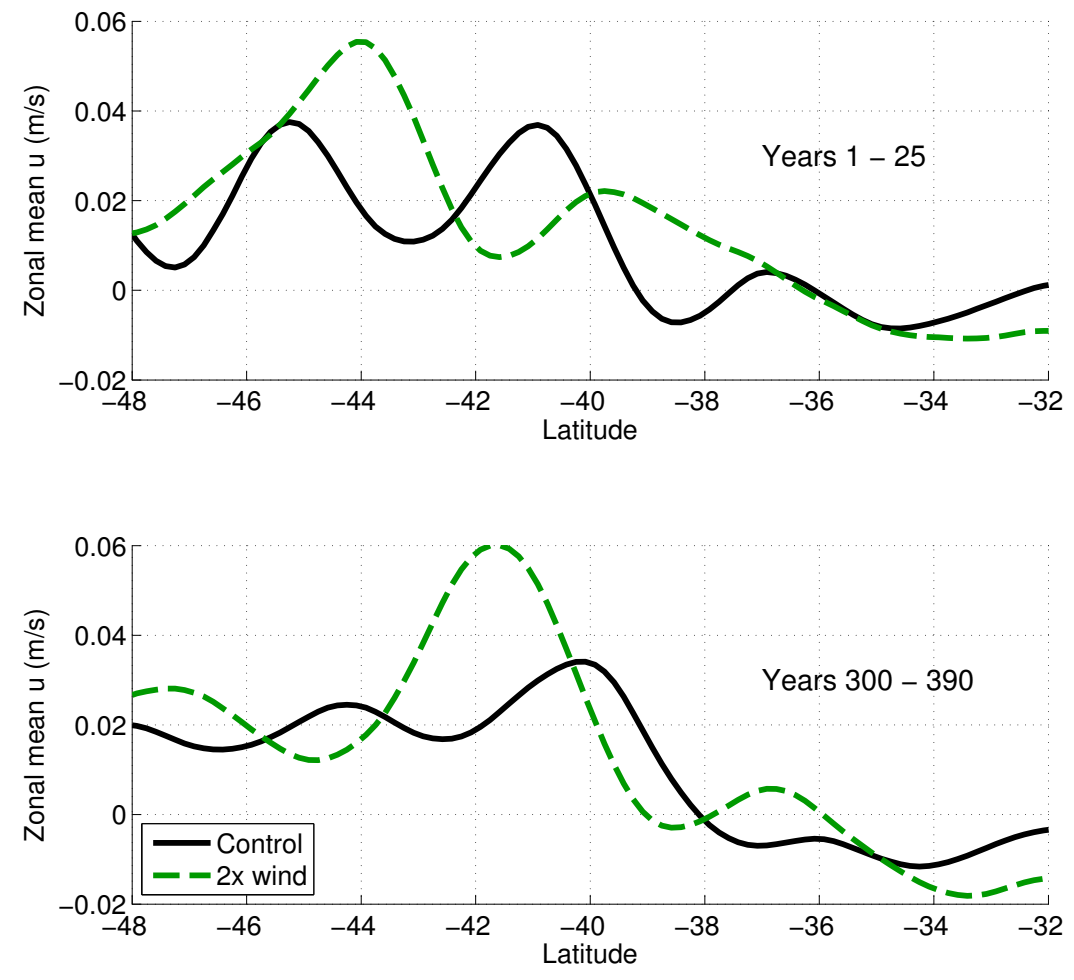

FIG. 5.4. Depth-integrated zonal mean zonal velocity versus latitude for both the control (solid black lines) and doubled wind (green dashed lines) cases for two different averaging periods. (Top) the first 25 years following the step change in wind stress and (bottom) over years $300-390$. 
just downstream of the channel opening. The north-south current structure in the western boundary also bears the imprint of alternating mesoscale jets (see section 5.4). The maximum southward extension of the circumpolar current contours increases under enhanced, sustained wind stress. As such, the stationary eddy compensation pathway is still be relevant in the fine-resolution model. Note that the meander of the $20 \mathrm{~Sv}$ contour, for example, can be much larger in any given annual mean than the long-term mean position (Figure 5.5). Both the variability and the mean of the maximum southward extent of the $20 \mathrm{~Sv}$ contour increase with stronger wind stress.
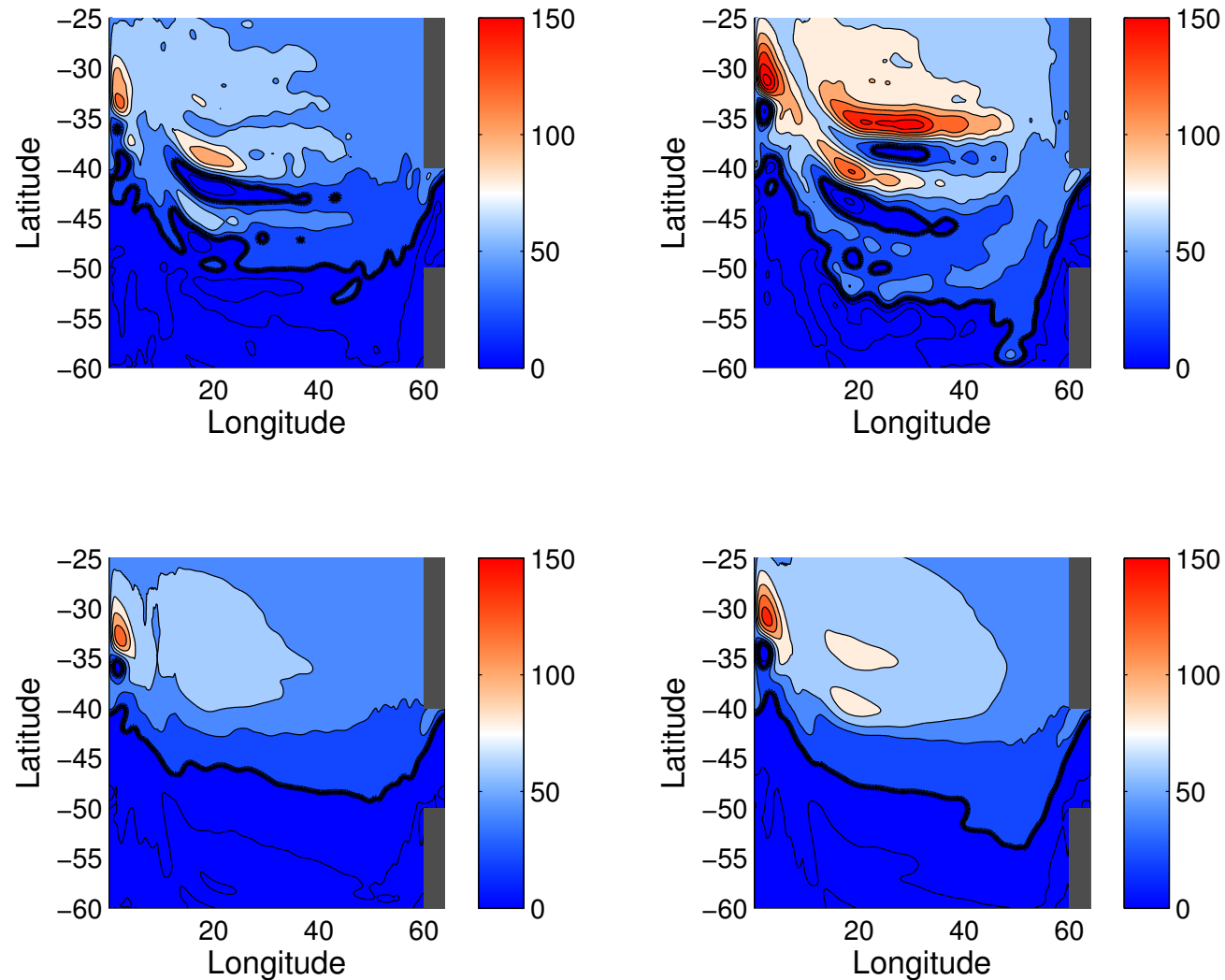

FIG. 5.5. Barotropic streamfunction for the control (left column) and doubled wind (right column) cases. The top row is an annual mean at year 360 and the bottom row is an average over years 330 - 360. The thick black line denotes the 20 Sv contour. 


\subsubsection{Overturning circulation}

Next, we examine the response of the meridional overturning circulation to increased wind stress. As in the coarse resolution case (Figure 4.6), the Eulerian mean circulation is driven by Ekman transport near the surface, downwelling along the narrow northern flank of the circumpolar current, and a much broader return flow to the south. The eddy-induced circulation is concentrated in the top $1000 \mathrm{~m}$ of the ocean and is surface intensified. The residual circulation is poleward right at the surface but is equatorward within much of the interior. Increased wind stress does speed up both the Eulerian mean and the eddyinduced circulations. However, since the responses of the Eulerian mean and eddy-induced circulations have different vertical structures, the changes in the two circulations do not cancel each other out (Figure 5.6). The residual overturning circulation is indeed sensitive to changes in surface wind stress, which is inconsistent with the strictest interpretation of the eddy compensation hypothesis (see section 1.3).

Using the maximum overturning strength at $42{ }^{\circ} \mathrm{S}$ as a metric of MOC strength, we see that the overturning circulation strengthens by around $60 \%$ within the first year, but shows no significant long-term adjustment following that. By component, the mean circulation increases by about $90 \%$, and the eddy-induced circulation, which has an opposite sense of circulation compared to the Eulerian mean, strengthens by $30 \%$. The difference shows up in the residual circulation. The standard deviation of the residual circulation increases by nearly a factor of four due to increased eddy variability.

\subsubsection{Eddy diffusivity of buoyancy}

Next, we examine the eddy diffusivity field using a rather simple approach. The meridional eddy diffusivity of (potential) temperature may be written as

$$
K_{T}=-\frac{\overline{v^{\prime} T^{\prime}}}{\partial_{y} T},
$$



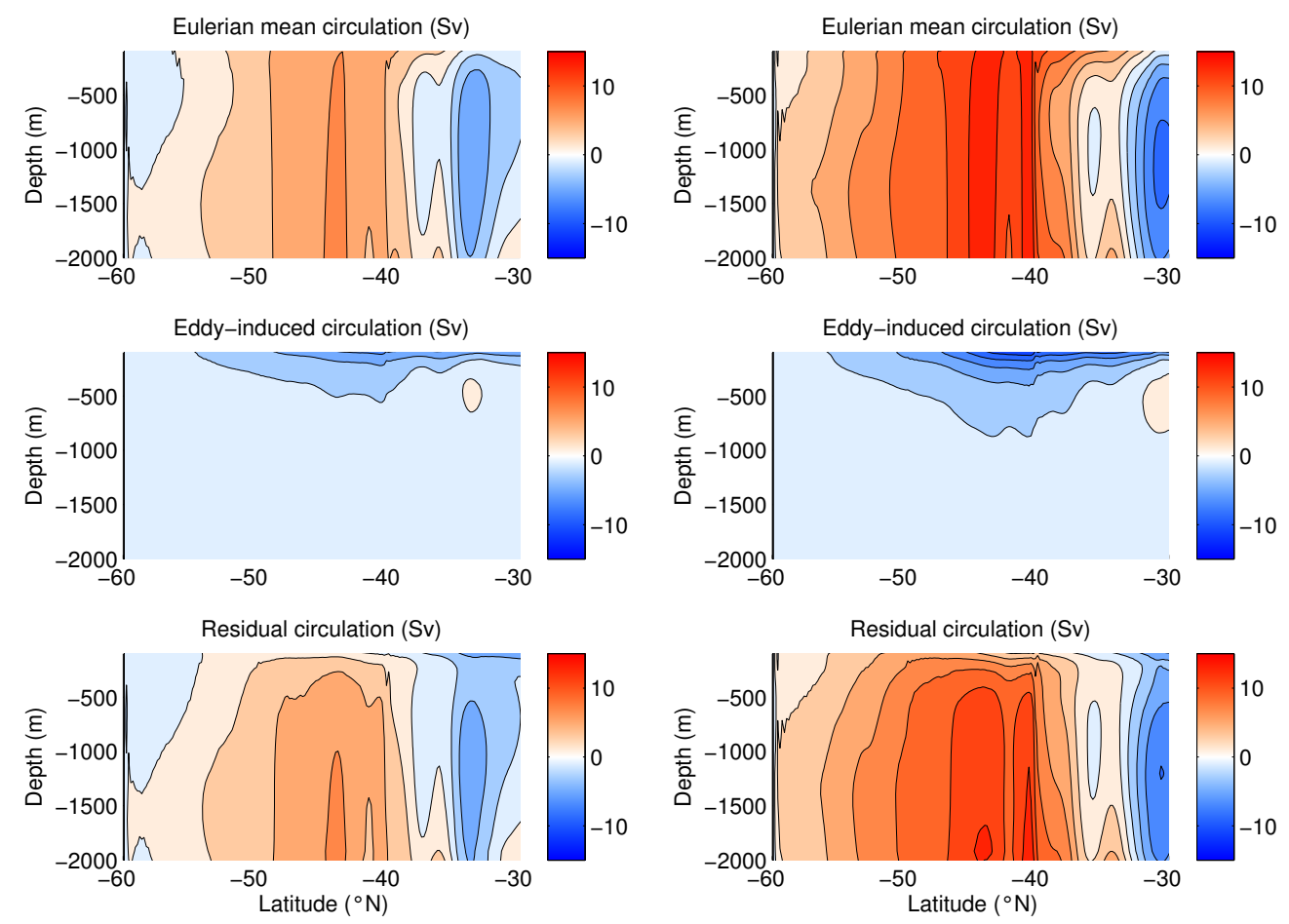

FIG. 5.6. Zonal mean meridional overturning circulation 350 years after the profile switch for the control (left column) and doubled wind (right column). Shown are the annual average Eulerian mean (top row), eddy-induced (middle row), and residual circulations (bottom row).

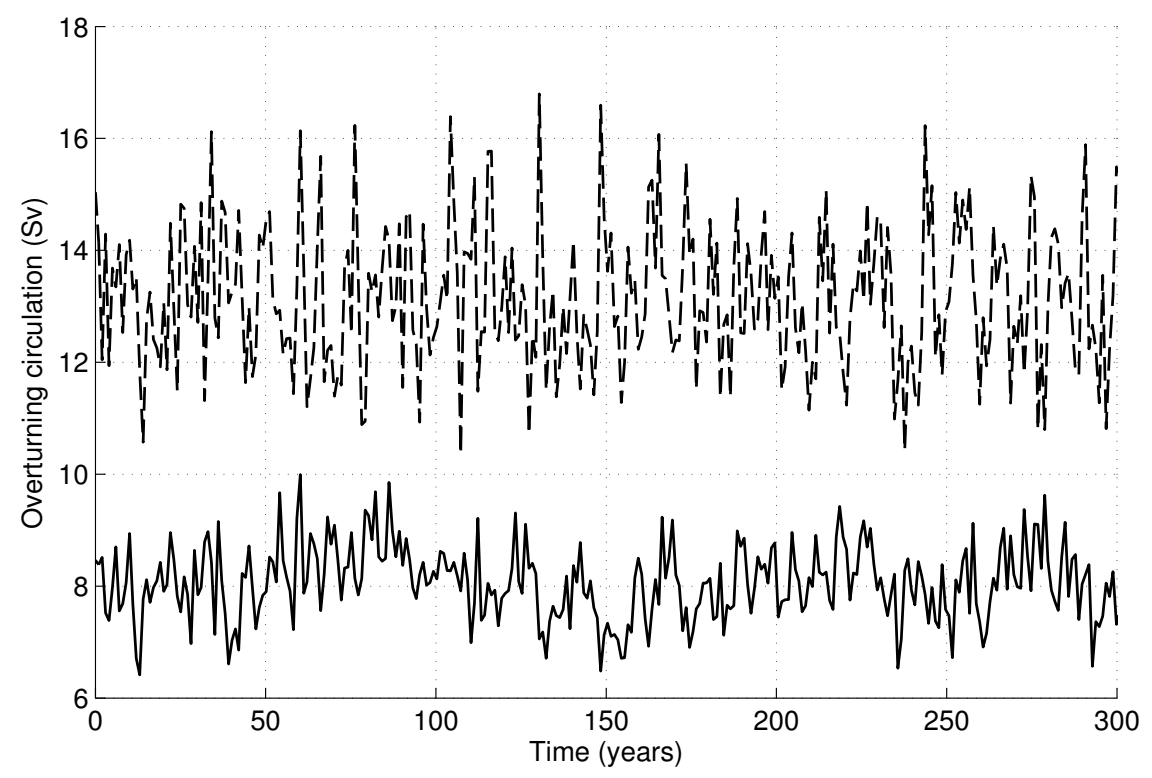

FIG. 5.7. Time series of the meridional overturning circulation for the control (solid) and doubled wind (dashed) cases, diagnosed as the maximum value of the residual circulation at $42{ }^{\circ} \mathrm{S}$. 
where the eddy flux $\overline{v^{\prime} T^{\prime}}$ is diagnosed from the model fields via the relationship $\overline{v^{\prime} T^{\prime}}=$ $\overline{v T}-\bar{v} \bar{T}$, and the overline represents a time average. We use a simple difference approximation for the temperature gradient across the channel, $\partial_{y} T \approx \Delta T / \Delta y=\left(T_{n}-T_{s}\right) / L_{y}$, where $T_{n}$ is the zonal mean temperature across the northern edge of the channel, $T_{s}$ is taken across the southern edge of the channel, and $L_{y}$ is the channel size in the meridional direction. Any particular annual mean map of $K_{T}$ is rather noisy. However, if we take the mean of many annual mean snapshots, a smoother picture emerges. The average value of $K_{T}$ (with $90 \%$ confidence interval) in the channel during the first 360 years of the simulation is $K_{T}=(2210 \pm 20) \mathrm{m}^{2} / \mathrm{s}$ in the control case and $K_{T}=(2690 \pm 30) \mathrm{m}^{2} / \mathrm{s}$ in the doubled wind case, i.e. a doubling of wind stress produces about a $20 \%$ increase in meridional eddy diffusivity. This is a very rough estimate that is only valid in the small neighborhood of parameter space occupied by our particular system parameters. The value of $K_{T}$ is largest around the northern flank of the channel opening, i.e. at the eastern and western edges of the basin along the topographic slope, where it can get as high as $5000 \mathrm{~m}^{2} / \mathrm{s}$. The sensitivity of $K$ to a change in wind stress can be approximated via linear expansion as

$$
\frac{\Delta K_{T}}{\Delta \tau} \approx-\frac{L_{y}}{\Delta T_{y}} \frac{\Delta\left(\overline{v^{\prime} T^{\prime}}\right)}{\Delta \tau}-\frac{K_{T}}{\Delta T_{y}} \frac{\Delta\left(\Delta T_{y}\right)}{\Delta \tau} .
$$

Using mean values from the simulation, we estimate a sensitivity of

$$
\frac{\Delta K_{T}}{\Delta \tau} \approx 3500 \frac{\mathrm{m}^{2} / \mathrm{s}}{\mathrm{N} / \mathrm{m}^{2}},
$$

where about $45 \%$ of the sensitivity comes from the eddy flux term and the other $55 \%$ from the temperature gradient term. The sensitivity of eddy heat diffusivity depends strongly on both of these factors. This estimate is roughly consistent with recently published estimates from other numerical studies (Abernathey et al., 2011).

\subsubsection{Potential vorticity}

In rapidly rotating, strongly stratified geophysical flows, large-scale circulation is heavily constrained by potential vorticity conservation. In particular, in a two-layer model of such a 
system, the response of the density structure to changes in surface forcing involves potential vorticity (Straub, 1993). Stronger winds enhance equatorward Ekman transport, which tends to steepen isopycnal tilt. This tilt stretches columns on the poleward side of the bottom layer and compresses them on the equatorward side. Eventually the PV gradient reverses sign and baroclinic instability is triggered, setting up an eddy flux that tends to flatten isopycnals back out. Since the reversal of a meridional PV gradient is only a necessary (not sufficient) condition for baroclinic instability in simple models of this process, there must be other conditions that are met to allow this instability to persist. However, practical experience suggests that PV gradient reversal is a fairly reliable indicator of the presence of instability.

First, we calculate potential vorticity using equation 4.8. Large-scale potential vorticity primarily depends on stratification, which is often assumed to be insensitive to changes in wind stress based on (fairly sparse) observations (Böning et al., 2008). In Figure 5.8 we show that PV (and therefore stratification) is at least weakly sensitive to stronger wind stress on centennial timescales. This is consistent with recent claims that eddy-permitting models are insensitive to changing wind stress on the timescale of a few decades (Meredith et al., 2012). Subtropical and high-latitude warming (induced by the gyre and circumpolar current meander, respectively) push the isopycnals down and gradually establish changes in the large-scale slope (see section 5.3).

The PV gradient changes near the surface in a banded fashion that may be related to the presence of small-scale alternating jets in the annual mean (Figure 5.8), though the presence of surface mixing complicates this interpretation. In the largely adiabatic interior, far away from the mixed layer, we find a region of PV gradient reversal indicative of instability in the channel (Figure 5.9). The sharp feature around $40{ }^{\circ} \mathrm{S}$ is an artifact of the transition from channel to gyre dynamics. The centennial-scale adjustment of the density structure is again evident. Stratification does change at least weakly with wind stress on time scales much longer than the available observational record. 

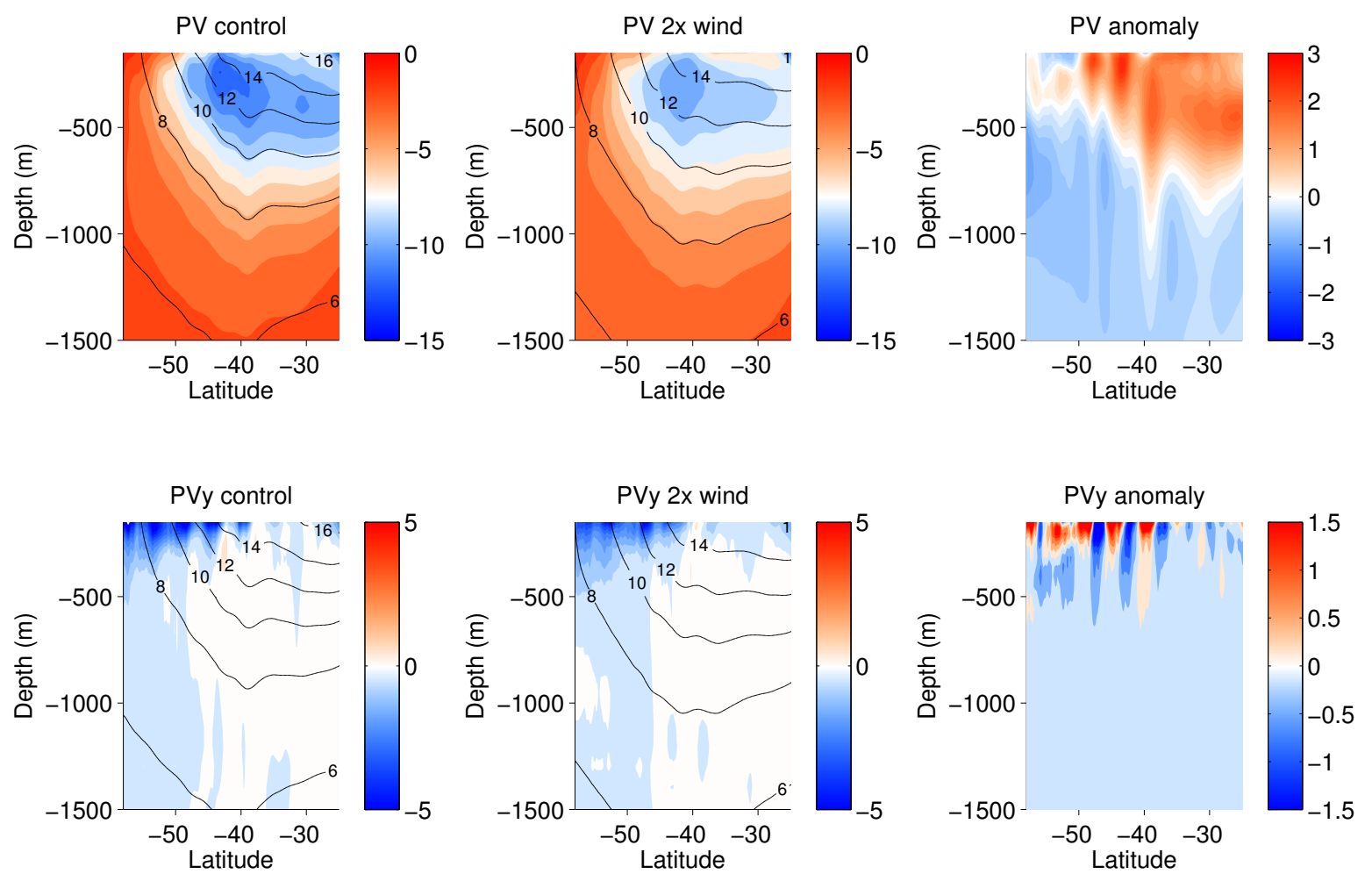

FIG. 5.8. Annual mean potential vorticity (top row, units of $10^{-11}(\mathrm{~ms})^{-1}$ and the meridional gradient of PV along zonal mean surfaces of constant density (bottom row, units of $10^{-16}$ $\mathrm{m}^{-2} \mathrm{~s}^{-1}$ ) around 350 years after the wind stress is increased. The anomaly (right column) is the difference between the doubled wind and the control fields. The top 150 meter layer is excluded. Temperature contours (solid black) are in steps of $2{ }^{\circ} \mathrm{C}$.
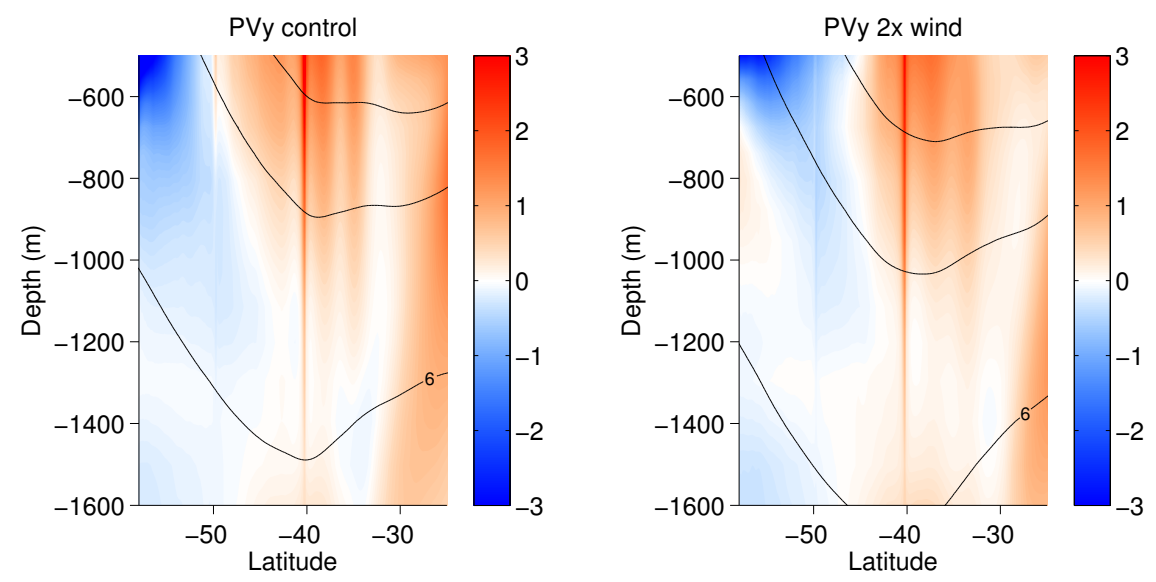

FiG. 5.9. Mean meridional gradient of PV along isopycnals in the interior (in units of $10^{-17}$ $\mathrm{m}^{-2} \mathrm{~s}^{-1}$ ). The average is taken over years $100-200$. Contours are in steps of $2{ }^{\circ} \mathrm{C}$. 


\subsubsection{Eddy fluxes of momentum, buoyancy, and PV}

In this section, we use quasi-geostrophic ( $Q G$ ) diagnostics to examine the pattern of meridional eddy fluxes. It should be noted that in the real Southern Ocean, some topographic features are on the same order of magnitude as the ocean depth, violating one of the assumptions of QG theory and limiting its applicability. Here we restrict our attention to the interior of the model domain away from the bathymetric features and below the mixed layer, where QG theory is expected to be applicable on the mesoscale and above. Here we calculate the EP flux vector and its divergence in order to examine the eddy fluxes in the model. In quasi-geostrophic theory, the meridional PV flux is given by $v^{\prime} q^{\prime}=\nabla \cdot \mathbf{E}$, where

$$
\mathbf{E}=\frac{1}{2}\left[\left(v^{\prime}\right)^{2}-\left(u^{\prime}\right)^{2}-\frac{\left(b^{\prime}\right)^{2}}{N^{2}}\right] \hat{\mathbf{i}}-\left(u^{\prime} v^{\prime}\right) \hat{\mathbf{j}}+\left(f_{0} N^{-2} v^{\prime} b^{\prime}\right) \hat{\mathbf{k}}
$$

Upon taking a zonal average and using no-slip boundary conditions at the edges of the domain, we can express the meridional eddy flux of potential vorticity anomalies as

$$
\overline{v^{\prime} q^{\prime}}=-\frac{\partial}{\partial y}\left(\overline{u^{\prime} v^{\prime}}\right)+\frac{\partial}{\partial z}\left(\frac{f_{0}}{N^{2}} \overline{v^{\prime} b^{\prime}}\right) .
$$

The above form suggests that we write down a vector of the form

$$
\mathbf{F}=-\overline{u^{\prime} v^{\prime}} \hat{\mathbf{j}}+f_{0} N^{-2} \overline{v^{\prime} b^{\prime}} \hat{\mathbf{k}}
$$

such that $\overline{v^{\prime} q^{\prime}}=\nabla_{x} \cdot \mathbf{F}$, where $\nabla_{x}=\left(\partial_{y}, \partial_{z}\right)$, the divergence in the meridional plane. The vector $\mathbf{F}$ is the EP-flux vector, which is a measure of flux associated with eddy transport. This flux may include contributions from stationary eddies, transient eddies, or both, depending on how the decomposition is carried out (here we calculate the total eddy contribution). The horizontal component of $\mathbf{F}$ is the (negative) of the meridional eddy flux of zonal momentum, and the vertical component of $\mathbf{F}$ is the meridional eddy flux of buoyancy, scaled by stratification and the local planetary vorticity. The EP flux vector can also be physically interpreted as a measure of wave activity. Here we use it to diagnose eddy fluxes.

The results are shown in Figure 5.10. First we note the difference in stratification between the control (top row) and doubled wind (bottom row) cases. At year 160, stronger 
wind has pushed isopycnals further into the interior and has pushed several of the outcrops southward. For example, the $7^{\circ}$ surface is pushed into the interior where it cannot outcrop to exchange buoyancy with the surface. It is effectively isolated from the atmosphere except for perhaps deep mixing. Equatorial stratification provides a strong constraint for the isopycnals as they extend northward out of the gyre region; the deepest surfaces especially shift by less than $50 \mathrm{~m}$ under strong wind conditions. Overall, the stratification exhibits a "kinked" Vshaped pattern; the mean slope changes rapidly at the channel-gyre transition latitude 40 ${ }^{\circ}$ S. The (large-scale average) sign of the slope reverses across this latitude, especially in the deep ocean. The V-shaped pattern is much more evident in the doubled wind simulation, and the sign of the eddy flux is dynamically consistent with the slope (i.e. the eddy flux is poleward where the slope is negative and equatorward where the slope is positive).

The eddy flux of zonal momentum $\overline{v^{\prime} u^{\prime}}$ is dominated by the signature of zonal mean jets. The flux represents both meandering of the large-scale current (stationary eddies) and the weather-like events that help to alter the jet profile (transient eddies). The large-scale density structure sets up the broad pattern of eddy buoyancy flux seen in the middle column of Figure 5.10. In the sub-polar and channel regions (where the slope is largely negative), $\overline{v^{\prime} b^{\prime}}$ is poleward. In the gyre region (where the slope is largely positive), $\overline{v^{\prime} b^{\prime}}$ is equatorward. Both of these fluxes tend to flatten out isopycnals. The meridional eddy flux of PV appears to be related to the maintenance of the jet structure. The convergence of eddy PV just south of $30{ }^{\circ} \mathrm{S}$ overlaps with the eastward jet and the divergence of eddy PV overlaps with the westward jet. The eddy fluxes both help to maintain the large-scale structure (especially the buoyancy fluxes) and set the smaller-scale structure variations in the annual mean picture.

\subsubsection{Potential vorticity homogenization}

In this section we discuss the tendency of eddy fluxes to create regions of constant potential vorticity and examine the implications for the large-scale density structure. We 


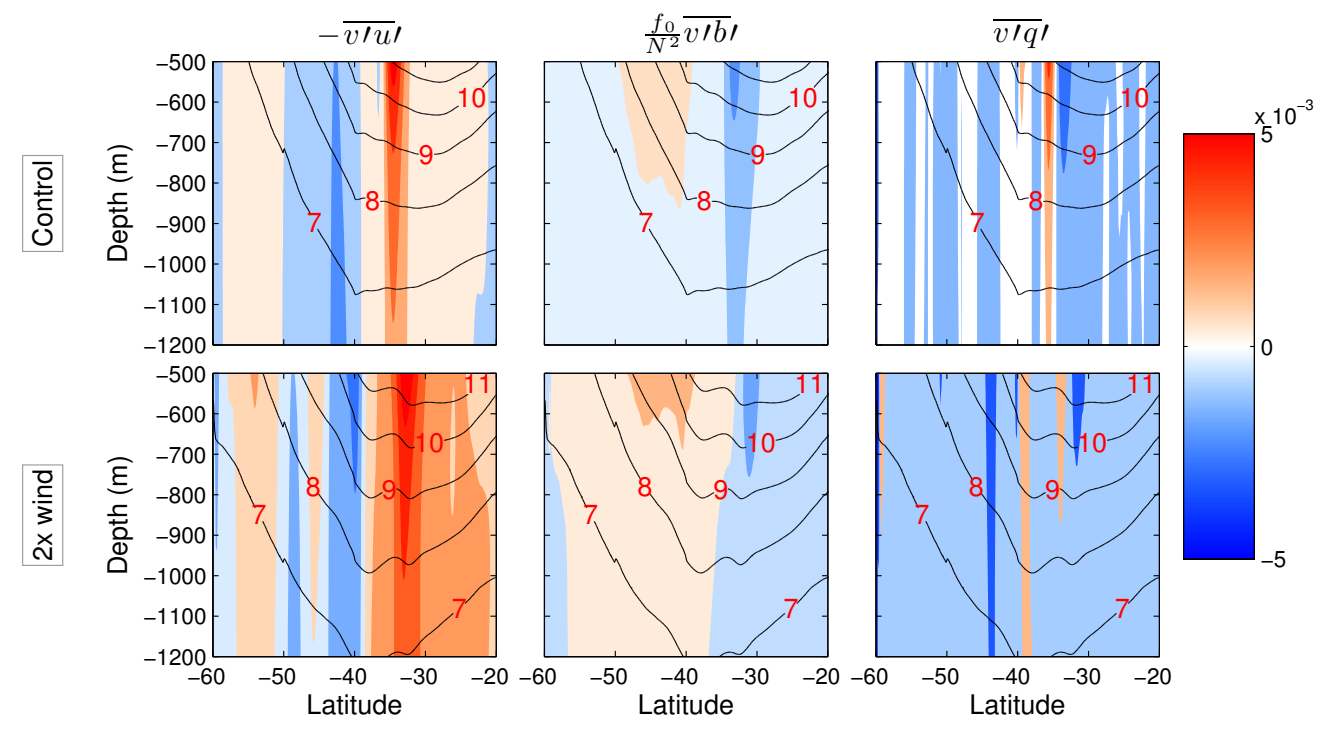

FIG. 5.10. Components of the EP flux vector $\left(\mathrm{m}^{2} / \mathrm{s}^{2}\right)$ and its divergence, representing annual mean eddy fluxes (color shading) of momentum (left column), buoyancy (middle column), and potential vorticity (right column). Both the control (top row) and doubled wind (bottom row) cases are shown. Isopycnals are shown as solid lines, and the mean is taken around year 160 in the simulation. Note that $f_{0}<0$ in the Southern Hemisphere.

begin with the quasi-geostrophic potential vorticity (QG-PV),

$$
q=\beta y+\nabla^{2} \psi+\frac{\partial}{\partial z}\left(\frac{f_{0}}{N^{2}} b\right),
$$

where $\beta$ is the meridional gradient of the Coriolis parameter, $N$ is the buoyancy frequency, and $b$ is the buoyancy field. The PV gradient is written

$$
\partial_{y} q=\beta+\frac{\partial}{\partial y} \nabla^{2} \psi+\frac{\partial}{\partial z}\left(\frac{f_{0}}{N^{2}} \frac{\partial b}{\partial y}\right)
$$

where we have used the typical convention that $N$ is a function of $z$ only. For large-scale dynamics the relative vorticity $\nabla^{2} \psi$ and its meridional gradient are typically small compared to the planetary and stratification terms, so the error incurred by neglecting the relative vorticity is small. In Transformed Eulerian Mean (TEM) theory, the evolution of zonal mean QG-PV can be expressed as

$$
\frac{\partial \bar{u}}{\partial t}=f_{0} \bar{v}^{\star}+\overline{v^{\prime} q^{\prime}}+\bar{F}
$$

where $\bar{u}$ is the zonal mean velocity, $\bar{v}^{\star}$ is the meridional component of the residual velocity, $\overline{v^{\prime} q^{\prime}}$ is the meridional eddy flux of potential vorticity, and $\bar{F}$ represents any forcing and 
dissipation terms. Under inertial $(\bar{F}=0)$, steady state $\left(\partial_{t} \bar{u}=0\right)$ conditions, we can relate the residual velocity to the eddy flux of PV,

$$
\overline{v^{\star}}=-\frac{\overline{v^{\prime} q^{\prime}}}{f_{0}}
$$

Furthermore, if we take the eddy-driven PV transport to be diffusive and downgradient (i.e. $\left.\overline{v^{\prime} q^{\prime}}=-\kappa \partial_{y} \bar{q}\right)$, then we can write equation 5.4 as

$$
\frac{\partial_{y} \bar{q}}{\bar{q}} \approx\left(\frac{\beta}{f_{0}}-\frac{\partial s}{\partial z}\right)
$$

In regions where the PV is homogenous $\left(\partial_{y} q=0\right)$, the vertical derivative of the slope is set, remarkably, by two geometric parameters that describe the spherical shape of the planet:

$$
\frac{\partial s}{\partial z}=\frac{\beta}{f_{0}}=\frac{2 \Omega a^{-1} \cos \left(\phi_{0}\right)}{2 \Omega \sin \left(\phi_{0}\right)}=a^{-1} \cot \left(\phi_{0}\right),
$$

where $a$ is the radius of the planet and $\phi_{0}$ is the reference latitude. With this rather unique result in mind, we define a "PV homogenization ratio":

$$
r=\frac{\overline{\partial_{z} s}}{\beta / f_{0}}
$$

where the overline denotes a vertical average. Note that $r<1$ is "under tilted" with respect to the equilibrium $\beta / f_{0}$ and $r>1$ is "over tilted" with respect to the equilibrium slope shear. In regions of inhomogeneous PV where $r<1$, the planetary effect dominates the stratification (as expressed through the change in slope with depth). In regions of inhomogeneous PV where $r>1$, the stratification overwhelms the planetary constraint.

Using equation 5.6, we may construct a simple bulk measure of homogenization by using a finite difference approximation,

$$
\frac{\Delta s}{\Delta z}=-\frac{1}{H} \frac{M^{2}}{N^{2}}=\frac{\beta}{f_{0}},
$$

where $H$ is a vertical scale and we have replaced the slope with a ratio of buoyancy frequencies. As such, $M^{2} \propto \beta H\left|f_{0}\right|^{-1} N^{2}$. In Figure 5.11, we plot several values of $M^{2}$ and $N^{2}$ 
from the channel region (below the mixed layer and above the topography). Simple scaling suggests that, if the PV is indeed homogeneous, the slope of the $M^{2}$ versus $N^{2}$ plot should be approximately $\beta H\left|f_{0}\right|^{-1}$, which is of order of magnitude $10^{-11+3+4}=10^{-4}$. This is consistent with the order of magnitude of the approximate slope in Figure 5.11. The large-scale PV in the interior of the channel is approximately constant following surfaces of constant density.

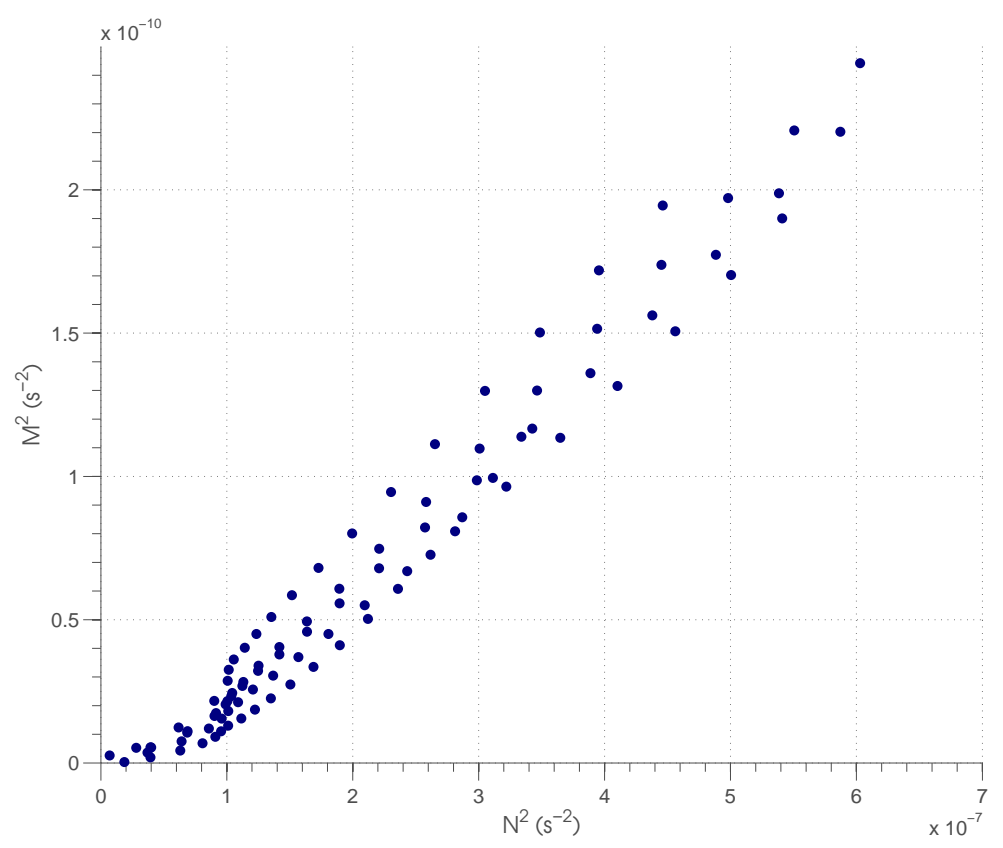

FIG. 5.11. Horizontal stratification $\left(M^{2}\right)$ versus vertical stratification $\left(N^{2}\right)$ for several different cuts across the channel.

We can also calculate the time series of the PV homogenization ratio (equation 5.7) averaged over the interior of the channel region, below the mixed layer, above the sill, and away from the side boundaries, where QG is expected to be a reasonably accurate description of the dynamics. Though the ratio $r$ is fairly close to unity in both the control and doubled wind cases (Figure 5.12), we do see some evidence of decadal scale adjustment. Within the first couple of decades of the experiment, $r$ in the doubled wind case develops a bias towards values less than unity $(r<1)$ while $r$ in the control case stays in the regime $(r>1)$. The decrease of $r$ in the doubled-wind case suggests that enhanced wind stress has either (i.) more effectively homogenized PV within the averaging volume or (ii.) changed the distribution of 
positive and negative $r$ anomalies associated with alternating jets. Spatial maps and zonal means of $r$ suggest that PV is indeed more effectively homogenized in the doubled wind case, despite the presence of alternating jets. On a centennial scale, the $r$ values in the control and doubled wind cases converge somewhat as the large-scale buoyancy structure and eddy fields approach equilibrium. We further explore the transient response of ocean circulation to changing wind stress in the next section.

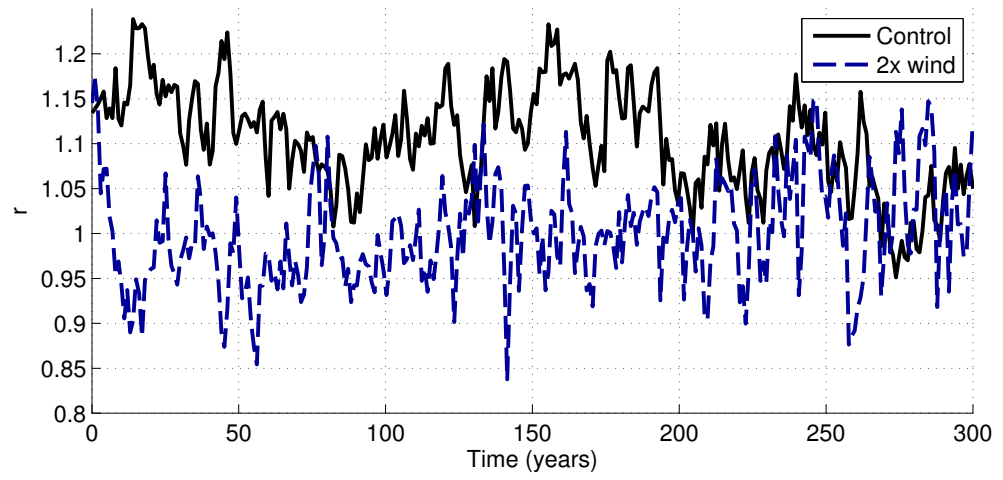

FIG. 5.12. PV homogenization ratio in the channel latitudes.

Finally, we examine the northward transport of heat and momentum as functions of latitude using the method discussed in section 4.2.6 as applied to both temperature and zonal momentum. We average six decades of model data to define the "zonal mean state" used in the definition of the stationary component. As in the coarse resolution experiment, the mean circulation in the channel approximately doubles under doubled wind stress conditions, likely from increased Ekman transport (Figure 5.13, left column). The increase in winddriven transport is largely canceled out by an increase in stationary eddy transport, i.e. from the meander of the stationary pattern of the circumpolar current). In terms of heat transport, eddy compensation seems to hold reasonably well in a quasi-equilibrium sense. However, this may partially be a consequence of our surface heat flux condition; the heat flux is constrained by the surface temperature profile. Even though the time mean values of the transient-eddy driven transport and net transport are not sensitive to wind stress in this experiment, every component of the heat transport (mean, stationary, transient, and 
net transport) exhibits much more interannual variability under enhanced wind conditions. Specifically, the standard deviation of annual mean transport across the channel increases by at least $40 \%$ for each component.

Unlike the buoyancy transport, the meridional transport of zonal momentum $(\overline{u v})$ is sensitive to the imposed change in wind stress (Figure 5.13, right column). The increase in poleward transport of zonal momentum is almost entirely eddy-driven and occurs primarily in the gyre region. Note that the eddy flux of momentum is not parameterized in the coarse resolution model, so the transport of zonal momentum by mesoscale eddies is unique to our eddy-permitting experiments. The momentum input at the surface is imparted downward by stress between fluid layers removed near the bottom by topographic form stress on the eastern side of the domain, in the fashion discussed by Munk and Palmén (1951).

\subsection{TRANSIENT RESPONSE}

In this section we explore the long-term (decadal) response of the eddying ocean to a large-scale change in wind forcing. In the coarse resolution simulation, Rossby waves dominate the transient adjustment. However, in an eddying simulation both Rossby waves and small-scale turbulence may affect the mean flow. We examine the zonal mean buoyancy structure, pycnocline depth, circumpolar transport, and the eddy diffusivity of buoyancy.

\subsubsection{Buoyancy structure}

In Figure 5.14, we plot the anomalous (doubled wind minus control) zonal mean buoyancy as a function of latitude and depth. The overall pattern is remarkably consistent with the coarse resolution case (see Figure 4.10), despite the fact that the low-resolution model does not parameterize the eddy flux of momentum. Note that the inclusion of the KPP 

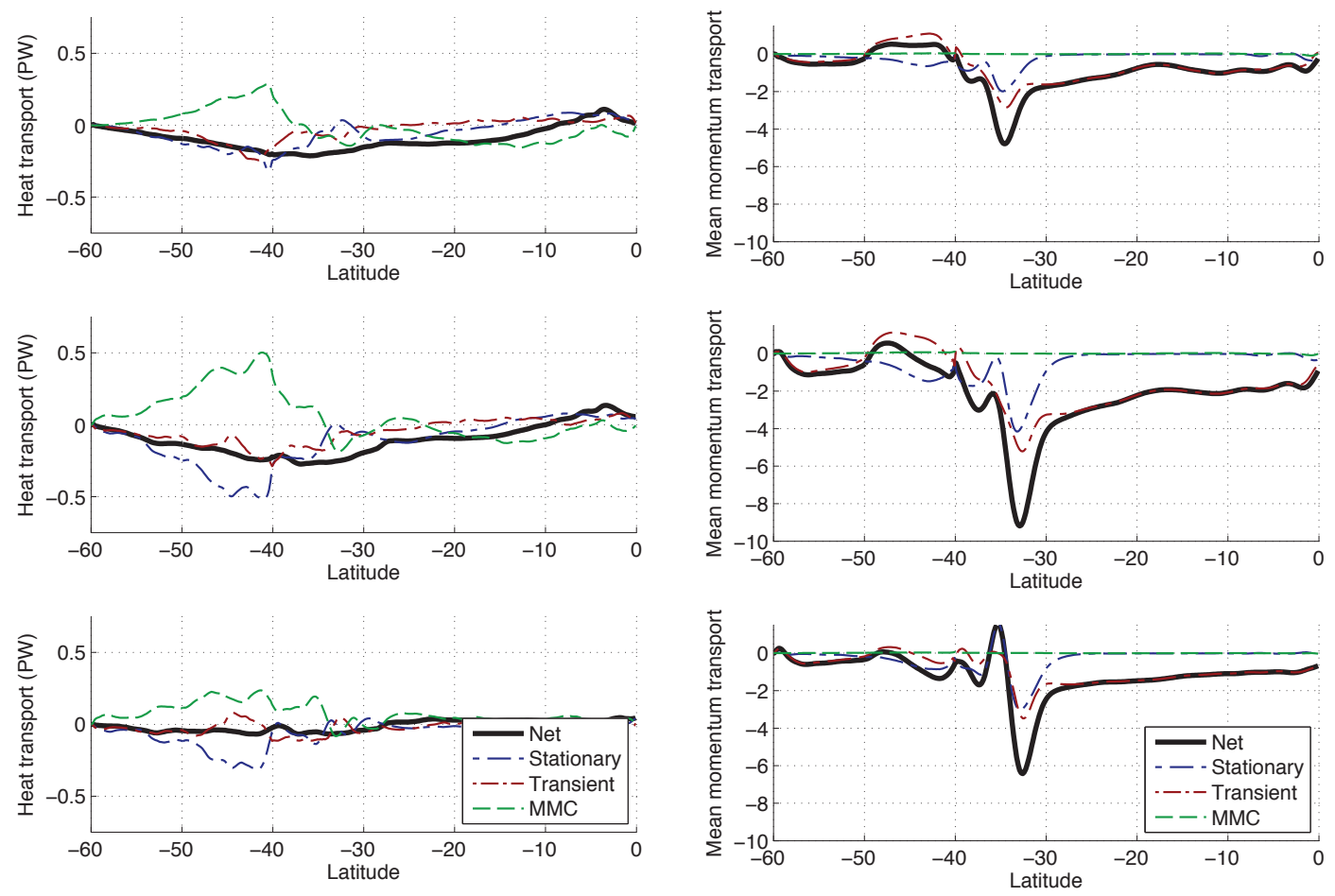

FIG. 5.13. Northward heat (left column) and eastward momentum (right column, $10^{7} \mathrm{~m}^{2} / \mathrm{s}^{2}$ ) transport for the control (top row), doubled wind (middle row), and anomalous (bottom row) fields.

package in both the coarse and eddy permitting models greatly improves the consistency between the two configurations.

Over the course of the first year, a cold anomaly develops at the surface due to enhanced Ekman transport, though it does not penetrate as far into the interior as in the coarse resolution case. Throughout the next decade the interior ocean warms at both high and low latitudes in a weakly banded pattern where the warming seems to be concentrated in the channel latitudes. In the coarse resolution case, the warming instead peaks at high northern latitudes. It may be that small-scale turbulence diminishes the extent of the stationary wave's meander. As the flow becomes more non-zonal, it becomes unstable to mesoscale turbulence and dumps energy into zonal jets instead of into a meandering mean flow. The agreement between coarse and eddying models improves in the following decades. By year 
100, we see two distinct warming regions - one at high southern latitudes and the other in the latitude band of the gyre. The low-latitude warming is sharper in the eddy permitting case.
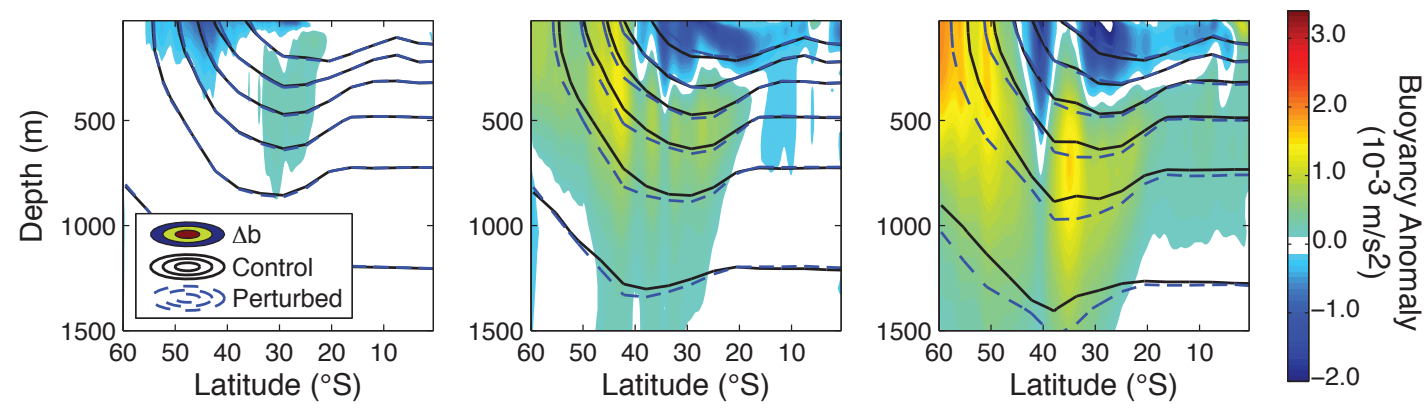

FIG. 5.14. Zonal mean anomalous (doubled wind minus control case) buoyancy structure as a function of latitude and depth at one (left), ten (middle), and 100 years (right) after the step function change is applied in the eddy permitting model. The lines are zonal mean isotherms (every $2{ }^{\circ} \mathrm{C}$ up to $16{ }^{\circ} \mathrm{C}$ ) from the control (solid) and doubled wind (dashed) experiments.

We can better understand the warming features by examining time series at southern hemispheric high latitude, southern hemispheric low latitude, and northern hemispheric high latitude (Figure 5.15). We see that the high-latitude warming increases relatively rapidly over the first few decades, which is consistent with a slow change in the standing wave pattern of the circumpolar current. The low-latitude pattern is much slower, increasing in a roughly linear fashion on a centennial scale due to gyre spin-up and the long-term adjustment of the buoyancy structure. The temperature anomaly in the Northern Hemisphere high latitudes displays much less interannual variability than that in the Southern Hemisphere, but there is still evidence of centennial-scale warming.

\subsubsection{Pycnocline depth}

The global pycnocline depth (given by equation 4.9) can be used as a measure of largescale adjustment. In Figure 5.16, we see that the control case is indeed not in thermodynamic 

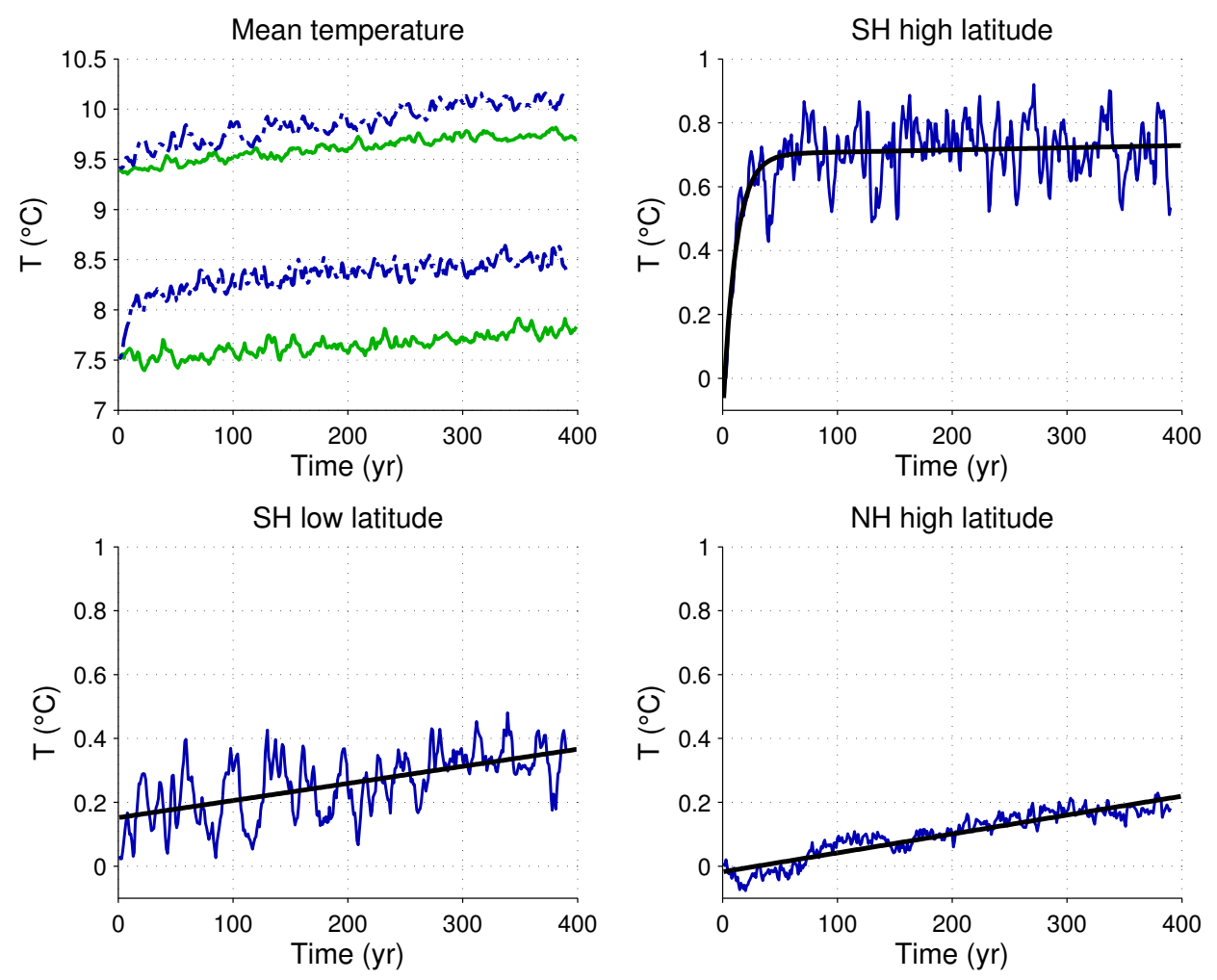

FIG. 5.15. (Top left) mean temperature time series for the control (green solid) and doubledwind (blue dashed) cases. Anomalous (doubled wind minus control) temperature time series at high southern latitudes (top right), low southern latitudes (bottom left), and high northern latitudes (bottom right). The black solid lines are simple fitting functions, which highlight long-term adjustment patterns (exponential in the top right, linear on the bottom row). 
equilibrium, as expected. Were it in equilibrium the pycnocline depth would be nearly constant with time. The pycnocline deepens for the first century and begins to shoal over the next several hundred years. In the doubled wind experiment, enhanced conversion of cold, high-latitude water to warm, low-latitude water in the Southern Hemisphere pushes the pycnocline deeper than in the control case, but only by a few percent. The transition from deepening to shoaling of the pycnocline is delayed by about a century compared to the control case, and some weak, centennial scale adjustment is evident in the centuries following the transition. Enhanced eddy activity (i.e. that permitted by the finer resolution grid) does not cancel out the increased wind-driven transport entirely, but it does mute the response of the pycnocline compared to the coarse resolution case. Assuming that the permitted eddies are indeed more nonlinear than their parameterized counterparts, the results shown in Figure 5.16 are broadly consistent with the predictions of our simple model from Chapter 2, wherein greater eddy nonlinearity shortens the pycnocline response timescale (i.e. a couple centuries compared to several millennia in the coarse resolution case) and weakens the magnitude of the adjustment (i.e. a few percent compared to around 10-20\% in the coarse resolution case).
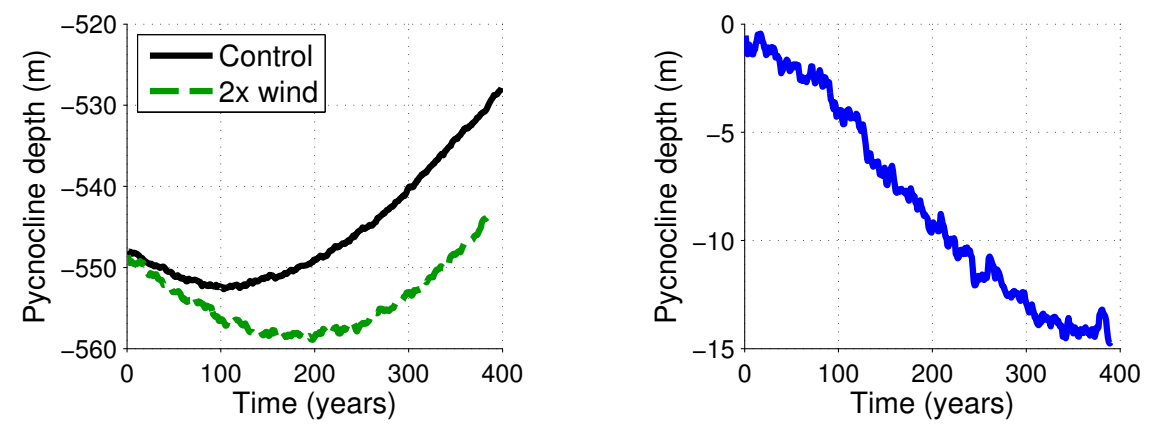

FIG. 5.16. Time series of pycnocline depth (left) and anomalous depth (doubled wind minus control, right) in the eddy-permitting experiment.

\subsubsection{Circumpolar transport}

Since a large portion of the energy imparted by the winds end up in the eddy kinetic energy as opposed to that of the mean flow, the circumpolar transport in the eddy-permitting 
model (Figure 5.17) is weaker than that seen in the coarse resolution case (Figure 4.17). There is a weak, century-scale linear slow down of the transport strength in both the control $(-1.6 \pm 0.4 \mathrm{~Sv} /$ century) and doubled wind $(-2.3 \pm 1.0 \mathrm{~Sv} /$ century) cases. The anomalous transport features a weak linear slow-down as well (-0.73 $\pm 0.7 \mathrm{~Sv} /$ century), though since the trend is small it is difficult to confidently distinguish the slope from zero (specifically, the error reported here is at the $95 \%$ confidence level. The slope cannot be distinguished from zero at the $99 \%$ level or above). The slow-down likely reflects the century-scale adjustment of the buoyancy structure as the across-channel temperature gradient is slowly reduced at roughly the same rate in both the control and doubled wind runs. However, the mean transport is consistently about $35 \%$ higher in the doubled wind case compared to the control case, so by this metric the circumpolar transport is indeed sensitive to wind stress, contrary to the eddy saturation hypothesis. The transport increase is established well within the first year, suggesting that fast barotropic and low-order baroclinic waves control the initial response. The (de-trended) transport is also more variable ( $\sigma$ increases by about 30\%) in the doubled wind case.
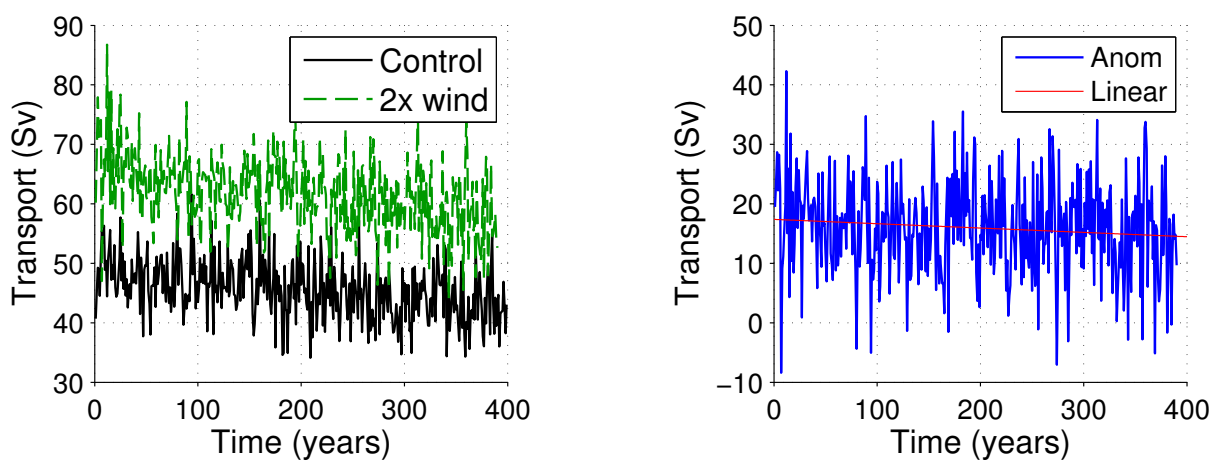

FIG. 5.17. Circumpolar current transport for both the control and doubled wind cases (left) and anomaly (right, doubled wind minus control). 


\subsubsection{Eddy diffusivity}

Finally, we discuss the transient behavior of the eddy diffusivity of temperature (buoyancy) and its components following the approach outlined in section 5.2.5. The diffusivity and the two factors that determine it (namely the eddy flux and meridional temperature gradient, through $\left.K=-\overline{v^{\prime} T^{\prime}} / \partial_{y} \bar{T}\right)$ are shown in Figure 5.18. Over the course of the first 2-5 years of the simulation (not shown), the (southward) eddy flux increases by about $35 \%$ in the doubled wind run, which is reflected in stronger eddy diffusivity. Over the first 100150 years of the simulation, the poleward eddy flux decreases while the temperature gradient weakens, which allows the diffusivity to remain elevated compared to the control case. Again we see that $K$ is indeed sensitive to increased wind stress, and its response is governed by the adjustment of both the eddy flux and large-scale temperature gradient in the channel. Note that both the eddy flux and the diffusivity display considerable interannual variability not shown in the smoothed-out time series, and as with other fine resolution model fields, this variability increases with enhanced wind stress ( $\sigma$ increases by about $40 \%$ for both components).

\subsection{INTERANNUAL VARIABILITY}

In this section, we examine the interannual variability of alternating jets in the gyre and channel regions of the model domain. The jets are essentially oceanic "storm tracks" along which anomalies propagate to the west across the basin on monthly to yearly timescales. The storm tracks themselves shift on annual to decadal timescales, and it is that variability that we attempt to study here. 

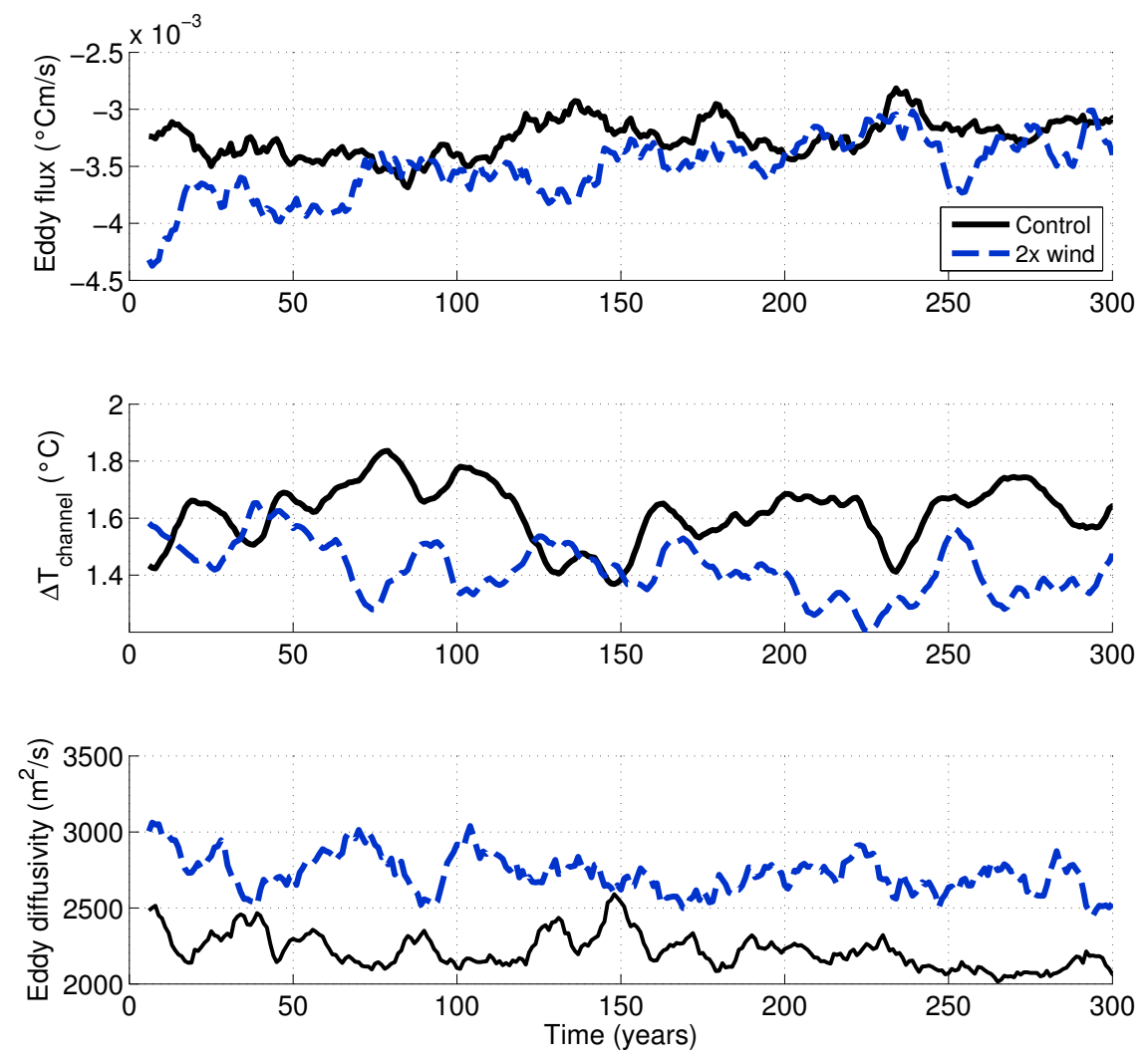

FIG. 5.18. Northward eddy flux of temperature (top), the temperature difference across the channel (middle), and the eddy diffusivity (bottom) for both the control and doubled wind cases. Each time series is passed through a decadal-scale running mean smoothing filter in order to emphasize the long-term behavior. 


\subsection{1 $\mathrm{EOF} / \mathrm{PC}$ analysis}

In order to better understand the interannual scale variability of an eddying ocean in a sector configuration, we apply empirical orthogonal function/principal component analysis (EOF/PC) to the 400 years of available annual-mean spatial maps (Lorenz, 1956). First, we apply EOF/PC analysis to anomalous, detrended sea surface height (SSH) data from both the control and doubled-wind simulations, i.e. the linear trend is removed from each grid point's SSH time series.

The first two EOF patterns are statistically significant and together can account for around $45 \%$ of the variability. EOF1 and EOF2 are very similar in appearance; they are orthogonal to one another (by definition and as verified by numerical integration) and constitute two phases of a meridionally-drifting pattern (Figure 5.19). The influence of the topography on the pattern is evident near the western side of the basin close to the sill. Both EOF1 and EOF2 are robust to record partitioning; they appear even if only the first or last half of the data is used, and they appear in both the control and doubled wind cases. The pair EOF1/EOF2 suggest the presence of a meridionally-propagating pattern in the gyre and channel latitudes. Hovmöller diagrams of SSH and zonal velocity show that the flow is concentrated in several narrow, filamentary tracks of alternating sign that drift in the meridional direction on a decadal timescale (see Figure 5.20). The jets are strongest in the channel latitudes between $50{ }^{\circ} \mathrm{S}$ and $40{ }^{\circ} \mathrm{S}$ but extend well into the gyre region.

\subsubsection{Drifting jets}

A Hovmöller diagram of sea surface height (SSH) with latitude reveals the meridional (mostly northward) drift of the jets on a decadal timescale (see Figure 5.20). The jets are zonally oriented in the ocean interior and cross the flat-bottomed part of the basin in 1-3 years (see Figure 5.21). The amount of variability seen in the western boundary current increases 

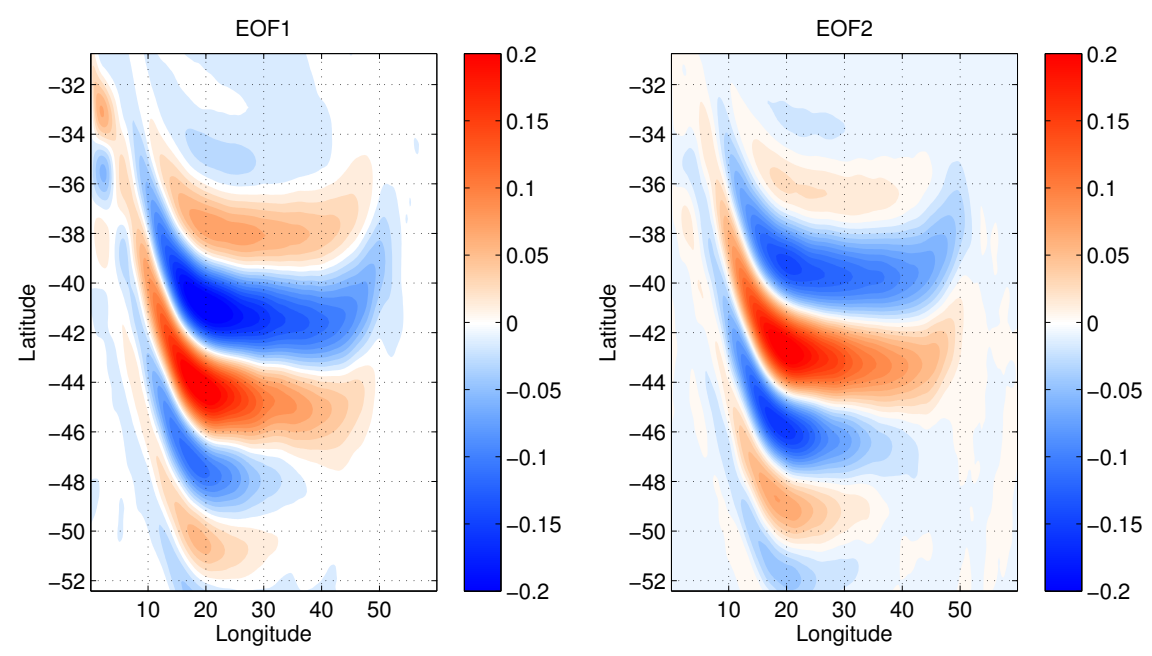

FIG. 5.19. First and second EOF patterns from the doubled-wind simulation. The patterns are statistically distinct from one another and are drawn from around 400 years of model data. The control case exhibits a similar pattern.

with latitude while the zonal anomalies become less prevalent. On interannual timescales, the anomalies form near the sill on the eastern side of the basin, propagate westward in the interior, deviate northward across the western sill, and collect in the western boundary current. On decadal timescales, the mean position where the anomalies tend to be found drifts meridionally. Now that we have described the propagation of these jets, we can attempt a brief explanation of their dynamics.

The jets are a manifestation of the complex interaction between wind stress, topography, and stratification. Here we discuss a possible mechanism for their formation and maintenance. The mean current typically follows contours of constant $f / h$, such that topographic steering is able to create non-zonal flows that are subject to instability (Kang et al. (1982), Marshall (1995a), Spall (2000)). In our model domain, the flow is strongly non-zonal and unstable on either side of the channel across the topographic ridge. Instability in these areas converts the kinetic energy of the mean flow into eddy kinetic energy and eddy length scales grow via inverse energy cascade. Once the eddy mixing length exceeds the topographic scale, zonal jets emerge that propagate westward throughout the interior of the channel (Thompson et al., 2010). Eddy mixing of PV in the jet flanks sets up an 
eddy-induced circulation that locally sharpens fronts in the upper water column, reinforcing the jet structure. At depths, eddies tend to homogenize PV along isopycnal surfaces, establishing vertically coherent density structures and strongly coupling horizontal and vertical stratification. Topographic steering deflects the jets northward across the downstream side of the still and into the region where the western boundary current is found.

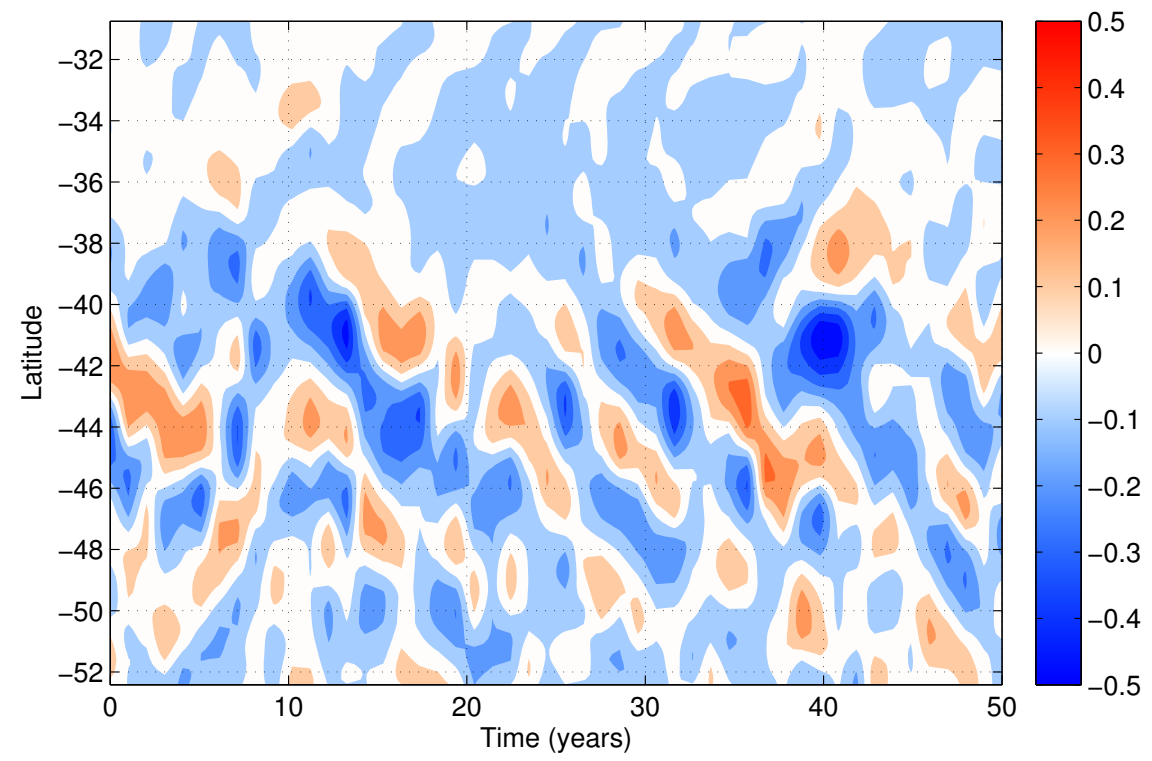

Fig. 5.20. Hovmöller diagram of a meridional section of SSH (m) at $20^{\circ} \mathrm{E}$ for the control case.

\subsection{3 $\beta$-plume}

A simple model, sometimes referred to as the $\beta$-plume, may help us get a better understanding of mesoscale jet dynamics. In a homogeneous ocean with topography, we may write the $\mathrm{PV}$ evolution equation as

$$
\frac{D q}{D t}=\frac{F}{h},
$$

where $q=f / h$ where $h=h(x, y)$ is the fluid depth, and $F$ represents vorticity forcing and friction (Vallis, 2006). Here we have neglected relative vorticity. Using linear drag for friction 

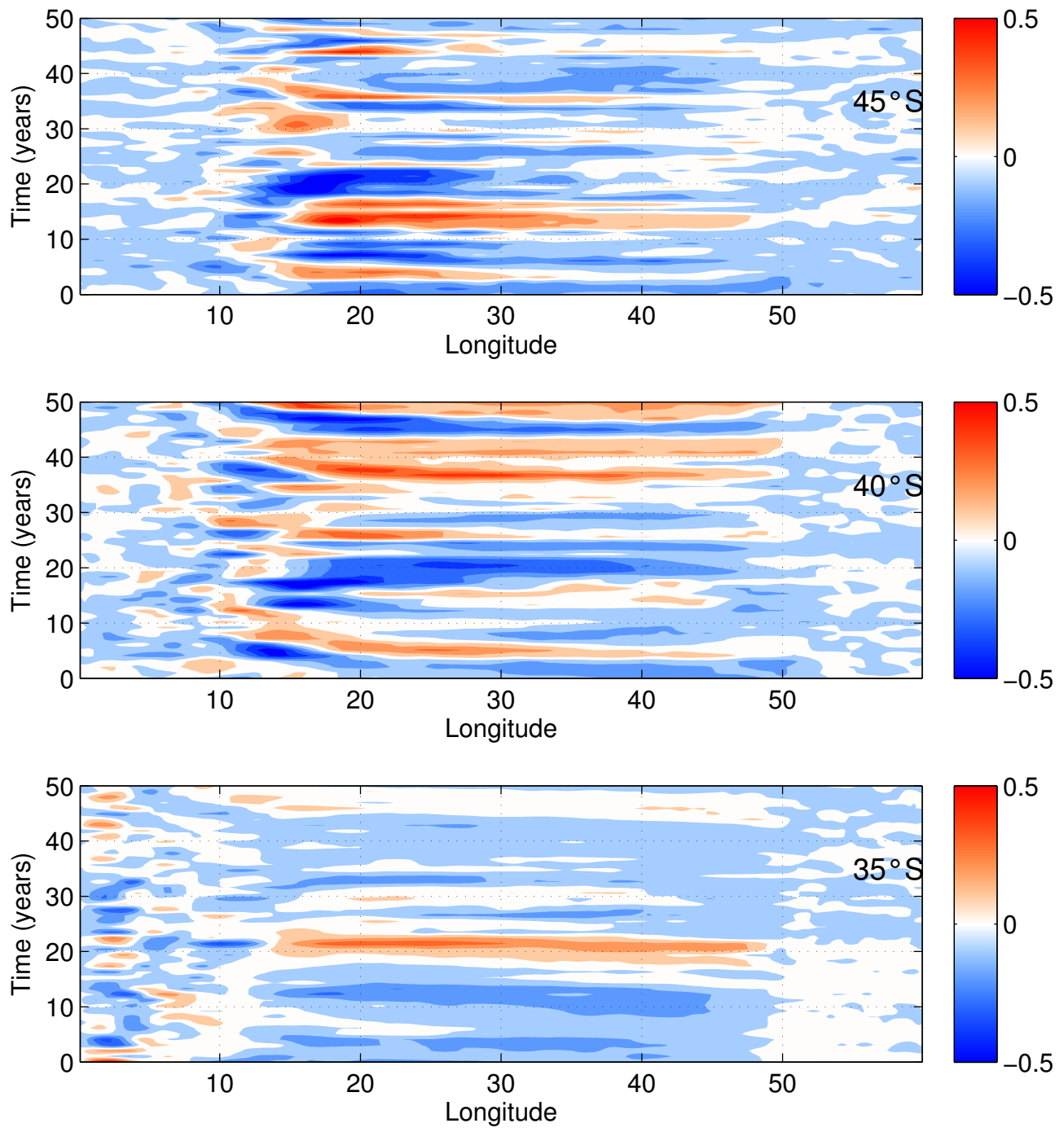

Fig. 5.21. Hovmöller diagram of SSH (m) versus longitude at three different latitudes. 
and wind-stress curl forcing, we have the advective form of the steady-state problem:

$$
J\left(\frac{f}{h}, \psi\right)=r \nabla^{2} \psi-\nabla_{z} \times(\tau / h),
$$

where $r$ is a friction parameter and $J(f / h, \psi)=\mathbf{U} \cdot \nabla \psi$ is the Jacobian. The pseudovelocity $\mathbf{U}=\mathbf{k} \times \nabla(f / h)$ is directed quasi-westwards, i.e. along surfaces of constant $f / h$ with high values of potential vorticity on the right. In the sector model configuration, the contours of $f / h$ curve equatorward when approaching the sill from the east (as freely propagating Rossby waves in a weak mean flow would). The contours of $\psi$ "diffuse" westward and converge towards the northwest of the mean flow (i.e. in the western boundary current in the sector model). There the $f / h$ contours are densely packed, and friction allows fluid to cross $f / h$ contours.

\subsubsection{Barotropic and baroclinic variability}

Sea surface height $\eta$ can be decomposed into a time-mean $\bar{\eta}$ and departures $\eta^{\prime}$ from the time-mean state, such that $\eta=\bar{\eta}+\eta^{\prime}$. We then have

$$
\overline{\eta^{2}}=\overline{\left(\bar{\eta}+\eta^{\prime}\right)^{2}}=\bar{\eta}^{2}+\overline{\left(\eta^{\prime}\right)^{2}}
$$

since $\overline{\eta^{\prime}}=0$ by definition. Using this result, we construct a measure of eddy variability as $\overline{\left(\eta^{\prime}\right)^{2}}=\overline{\eta^{2}}-\bar{\eta}^{2}$. Following Baker-Yeboah et al. (2009), we decompose sea surface height variability into baroclinic and barotropic components using the bottom pressure $P_{b o t}=$ $\bar{P}+P^{\prime}$. The baroclinic component of the sea surface height is given

$$
\eta_{b c}=\frac{\phi_{\bar{P}}}{g}
$$

where

$$
\phi_{\bar{P}}=\frac{1}{g} \int_{0}^{\bar{P}} \delta d P=-\int_{z(P=0)}^{z(P=\bar{P})}\left(1-\frac{\rho}{\rho_{0}}\right) d z
$$

and $\delta=\alpha(S, T, p)-\alpha(35,0, p)$ is the specific volume anomaly. The component of variability associated with changes in the density of the water column can be diagnosed from $\eta_{b c}^{\prime}=\eta-\bar{\eta}$. 
The component of variability associated with changes in the total mass of the water column (i.e. the barotropic anomaly) can be estimated using

$$
\eta_{b t}^{\prime}=\frac{P_{b o t}-\bar{P}}{\rho_{b} g}
$$

Much of the model's variability is consistent with bottom pressure anomalies, especially at high latitudes and in the eastern portion of the gyre (see Figure 5.22). In those locations the flow is largely independent with depth. There are many areas where the variability has a strong baroclinic component, such as along the equator and within the gyre-channel regions in both hemispheres. Again we see evidence of topographic steering on the western side of the basin across the sill.

Now we discuss the effect of increased wind stress on sea surface height (SSH) and sea surface temperature (SST) variability. There is a $32 \%$ increase in mean Southern Hemispheric SSH variability between the control and doubled-wind simulation measured about 100 years after the perturbation. Interestingly, there is also a $15 \%$ increase in SSH variability in the northern hemisphere. There is a $16 \%$ increase in SST variability and there is actually a $1 \%$ decrease in SST variability in the northern hemisphere. There is a significant increase in mesoscale variability after doubling the wind. This increase shows up in both hemispheres given sufficient time, but the increase is about twice as strong in the SH compared to the NH. The difference in mesoscale variability between the control and doubled run is statistically significant at the $95 \%$ level $(\alpha=0.05)$.

\subsection{DISCUSSION}

In both the coarse and eddy permitting runs, an increase in wind stress strengthens equatorward Ekman transport south of $30^{\circ} \mathrm{S}$ and sets up a cold anomaly near the surface in the channel. Over the course of the first decade, the contours of the ACC meander further southward downstream of the channel opening and heat up the subpolar region. 

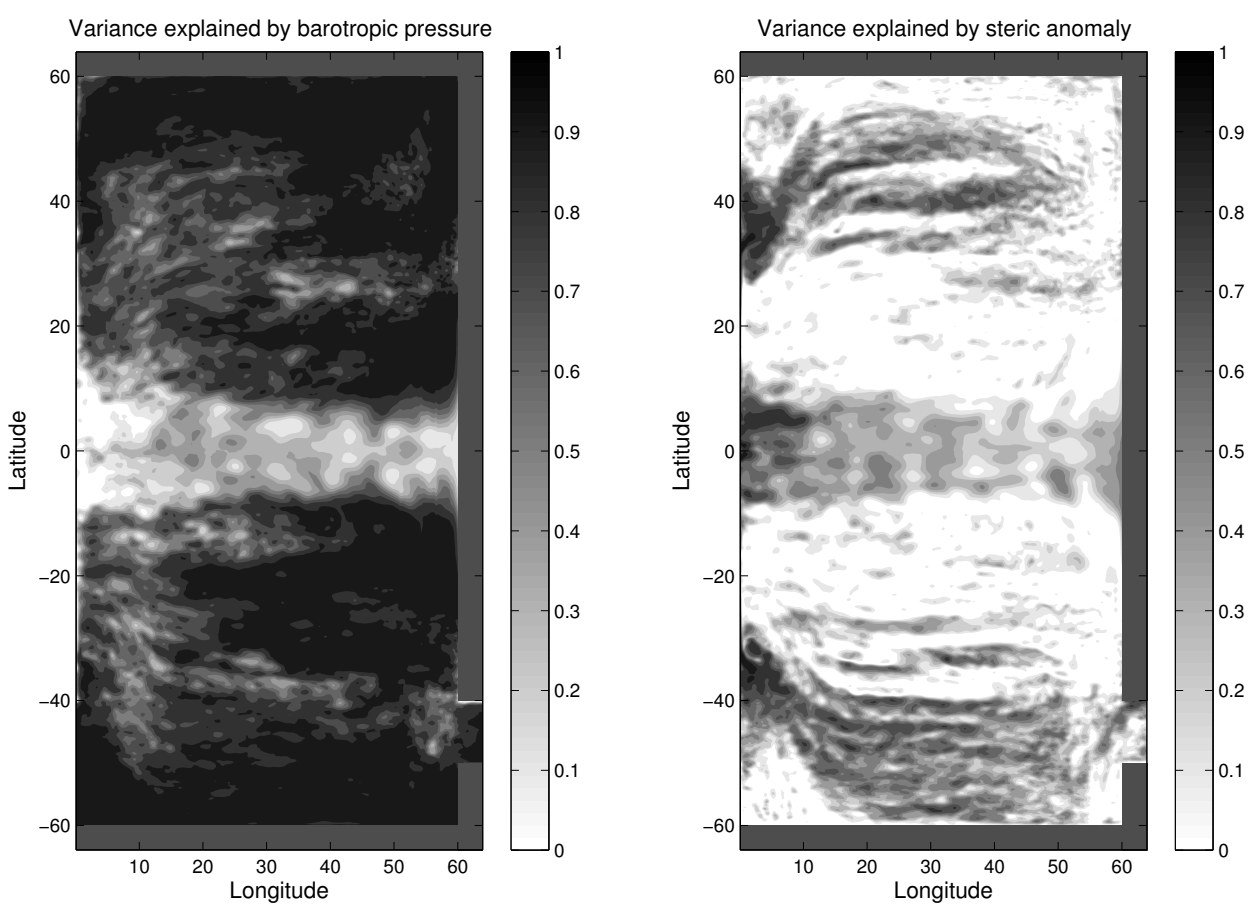

FIG. 5.22. Component of SSH variability $\left(r^{2}\right)$ congruent with barotropic (left) and steric (right) anomalies.

Throughout the next several decades, the subtropical gyre spins up, warming the ocean interior. However, the eddy permitting run hosts a great deal of interannual variability that impacts the dynamics and wind stress sensitivity. Much of the extra energy from strong winds ends up in mesoscale eddies, particularly those that form in the channel and gyre in the form of narrow, alternating jets.

The residual overturning circulation and stratification are at least weakly sensitive to wind stress on multi-decadal to centennial timescales, in part because the Eulerian mean and eddy-induced streamfunctions have different vertical structures and cannot completely cancel each other out. The wind-driven SO adjustment process has a local component (i.e. Eulerian versus eddy-induced circulation) and a global component (i.e. feedback between Southern Ocean stratification and northern basins through the global pycnocline). Small changes in the local SO pycnocline are modified on multi-decadal to centennial timescales by interactions with the rest of the ocean. Our model displays evidence of this long-term adjustment, which 
has until now not been explored in a primitive equation model. As such, when considering the long-term response of Southern Ocean circulation to decadal wind stress variability, interactions between the SO and the global ocean should not be neglected. Based on this reasoning, it is likely that the SO circulation and stratification will respond to the recent decadal-scale increase in wind stress over the next several decades to centuries. It is difficult to detect this response signal with the relatively short (and sparse) data record available at this time. A sustained observational program and more realistic numerical simulations are needed to better quantify the strength and nature of the wind-driven adjustment. 


\section{PERIODIC FORCING EXPERIMENTS}

The numerical step response experiments of the previous two chapters are useful for exploring decadal to centennial scale adjustment, but each experiment only offers one realization of the oceanic response in the first several years following a change in wind stress. In this chapter we attempt to better understand the interannual response of ocean circulation to changing wind stress. Using eddy-permitting horizontal resolution $\left(1 / 6^{\circ} \times 1 / 6^{\circ}\right)$, we conduct a set of periodic forcing experiments in which the maximum wind stress magnitude is given as a sinusoidal function of time (section 6.1). We also examine the effect of an additional meridional seamount on the response of mean and eddying circulation to changing wind stress (section 6.2). The periodic forcing approach affords multiple realizations of monthly and interannual adjustment, facilitating our efforts to understand windand buoyoancy-driven gyre/channel dynamics on those timescales.

\subsection{WIND STRESS MAGNITUDE}

Here we conduct a periodic forcing experiment in which the wind stress profile is smoothly and periodically transitioned via linear interpolation between the control and doubled wind profiles described in section 3.3. We employ the same buoyancy forcing and bathymetry used in the step response experiments. The time-dependent component of the forcing function is given by

$$
\tau_{t}(t)=-0.065 \cos \left(2 \pi \frac{t}{T}\right)+0.195
$$

where $T$ is the period, set to 5 years (60 months) in each experiment. Linear instability theory predicts a time scale for the growth of the most unstable mode (i.e. the Eady time scale) of about $T_{E}=L_{d} /(0.3 U) \approx 10^{5} /(0.3 \times 0.1) \mathrm{s} \approx 38$ days, where $L_{d}$ is the deformation radius and $U$ is the velocity scale (Vallis, 2006). As such, a five-year forcing cycle should be long enough to permit at least the fastest-growing eddies to develop. The forcing pattern 
as a function of latitude and time is shown in Figure 6.1. Note that the maximum wind stress occurs in the channel region. A natural extension to this set of experiments is to use forcing profiles with different values of $T$ and examine the lag between forcing and various circulation diagnostics. We leave that for future work.

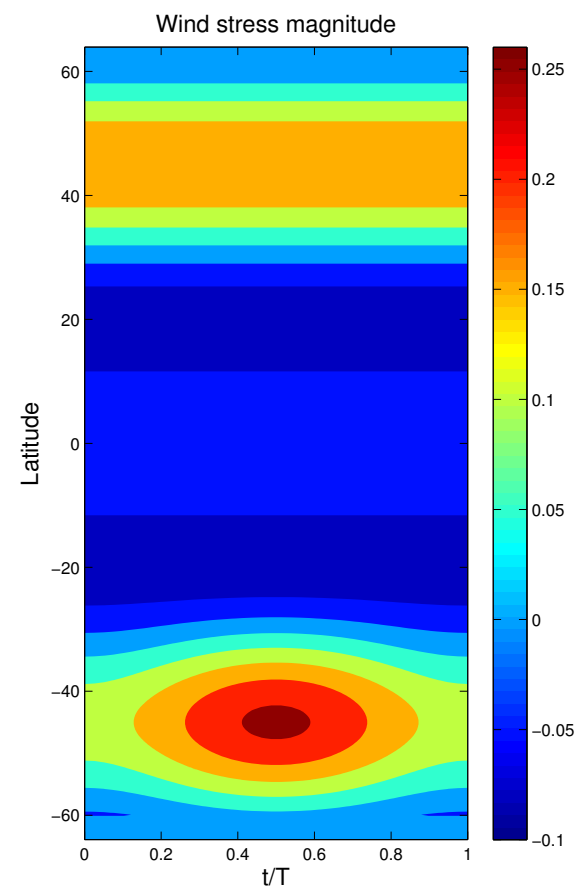

FIG. 6.1. Hovmöller diagram of wind stress magnitude across all model latitudes. The wind stress is zonally uniform and is periodic in time with period $\mathrm{T}$.

\subsubsection{Meridional overturning circulation}

First, we diagnose the maximum meridional overturning circulation in the channel region of the domain using monthly mean model fields. For each monthly mean field, we identify the maximum value in depth of the overturning circulation at $42^{\circ} \mathrm{S}$. As such, this metric may at times indicate the circulation strength at different depths. The results are shown in Figure 6.2, with the forcing represented by the black negative cosine curve in the center of the figure. The mean circulation is fairly well correlated with the forcing $\left(r^{2}=0.6\right)$ with no appreciable lag. Much of this correlation comes from the rapid, daily-timescale response of wind-driven 
Ekman transport near the ocean surface. Interestingly, the eddy-induced circulation shows little correlation with the wind stress forcing (maximum $r^{2}=0.3$ at a lag of 5 months, with forcing before circulation). The residual circulation mostly follows the Eulerian mean overturning $\left(r^{2}=0.8\right)$, with some variability introduced by the eddy circulation. Based on this metric of interannual oceanic response, eddy compensation does not appear to provide a strict constraint on the overturning circulation in the channel on interannual timescales.

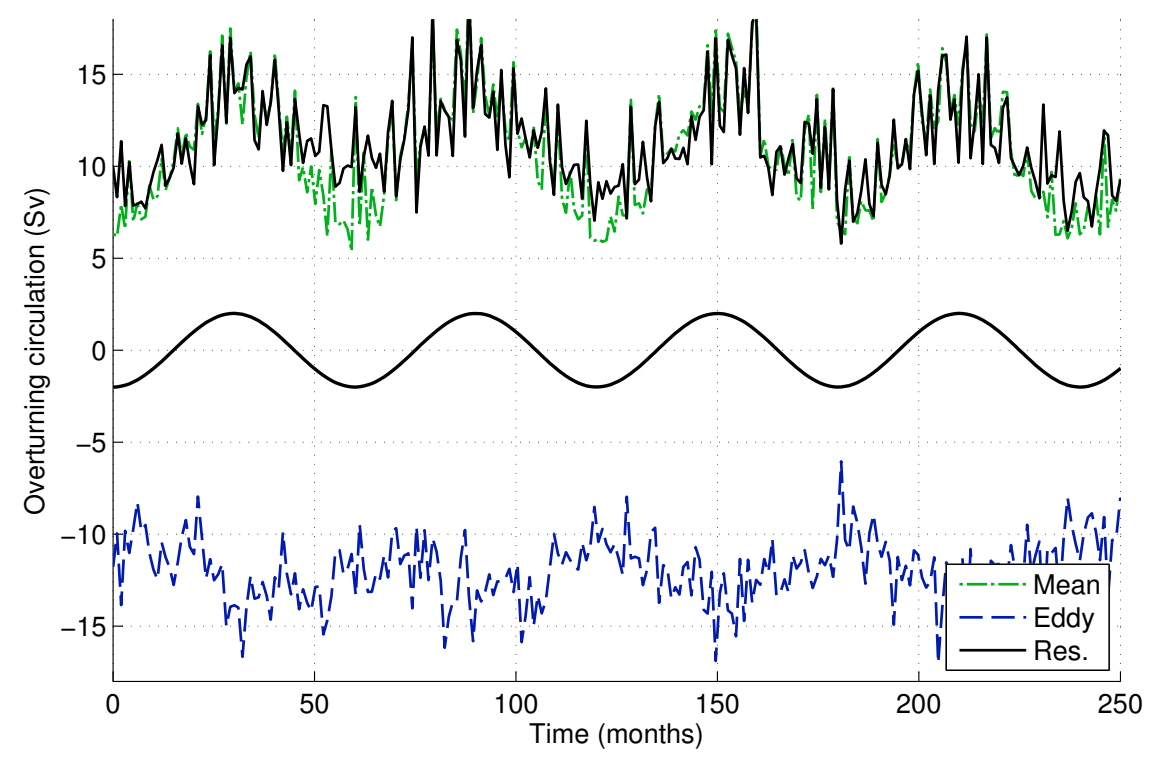

FIG. 6.2. Maximum meridional overturning circulation by component at $42^{\circ} \mathrm{S}$. The smooth black curve in the center represents the magnitude of wind stress forcing. The jagged black line is the residual circulation strength, the green dash-dot line is the Eulerian mean circulation, and the blue dashed line is the eddying circulation, including both stationary and transient components.

\subsubsection{Circumpolar current and EKE}

The additional power input provided by increased wind stress may end up in the kinetic energy of the mean circulation, the potential energy of the buoyancy field, or in the eddy kinetic energy. As such, increasing the wind stress by some factor may have a nonlinear effect on the flow. In Figure 6.3, we plot the standardized circumpolar transport (one measure of the energy contained in the mean flow) and the eddy kinetic energy EKE = 
$\frac{1}{2}\left[\overline{\left(u^{\prime}\right)^{2}}+\overline{\left(v^{\prime}\right)^{2}}\right]$, where $\overline{\left(u^{\prime}\right)^{2}}=\overline{u^{2}}-(\bar{u})^{2}$ and the overline denotes a monthly temporal average. The decorrelation timescale $b$ is estimated by fitting the autocorrelation curve of the deseasonalized time series to a decaying exponential $y=\exp (-t / b)$, and the number of independent measurements (i.e. degrees of freedom) of $f(t)$ (i.e. transport or EKE) is estimated by comparing the record length to the decorrelation timescale. We estimate that transport decorrelates in about two months and EKE in three months, giving us 127 independent ACC measurements and 83 independent EKE samples in the full record.

The wind stress and (smoothed) circumpolar current are reasonably well correlated $(r=$ 0.8), and composite analysis reveals a statistically significant (at the $95 \%$ level) bias of the mean transport towards smaller/larger values when the wind stress is especially weak/strong (see Figure 6.4). The difference between the positive composite mean and the negative composite mean transport is $0.59 \pm 0.5$ (standardized, 95\% CI). The (relatively meager) success of this particular composite analysis rests on the availability of a very large number of data points. It is difficult to detect a composite difference using the current observational record, which is rather sparse compared to available model data. Simple linear regression of transport $T$ versus wind stress $\tau$ suggests a wind stress sensitivity of $\Delta T / \Delta \tau=(115 \pm 70)$ $\mathrm{Sv} / \mathrm{N} / \mathrm{m}^{2}(95 \% \mathrm{CI})$, such that a change of $\Delta \tau=0.13 \mathrm{~N} / \mathrm{m}^{2}$ produces a change in transport of $\Delta T=(15 \pm 9) \mathrm{Sv}$, which is $12-48 \%$ of the mean transport (50 Sv in this simulation). This is consistent with the sensitivity exhibited by the high-resolution step response experiment in Chapter 5. Significant scatter in $T$ makes it difficult for a simple linear model to constrain the transport sensitivity. Assuming little change in scatter, increasing the degrees of freedom (by running the simulation longer) by a factor of 10 would reduce the margin of error from around $9 \mathrm{~Sv}$ to $3 \mathrm{~Sv}$.

While there is only a small correlation between wind stress and EKE $(r=0.3,14$ months lag), composite analysis does reveal a significant difference (at the $95 \%$ level) in mean EKE with wind stress. Stronger wind stress increases mean EKE, but with a maximum lag at 14 
months. Visual inspection of Figure 6.3 suggests that the peak in EKE does at times have a peak around one year after the wind stress maximum, but this does not seem to occur for every maxima. It may be the case that the eddy response depends on the mean state, which itself changes over the course of the simulation. Doubling the wind stress produces roughly a 30\% increase in EKE (averaged across the channel and full depth). Finally, circumpolar transport and EKE have a maximum correlation of $r=0.5$, with EKE lagging behind transport by 13 months. Additional simulated forcing cycles are needed to better address the accuracy and significance of these correlations.

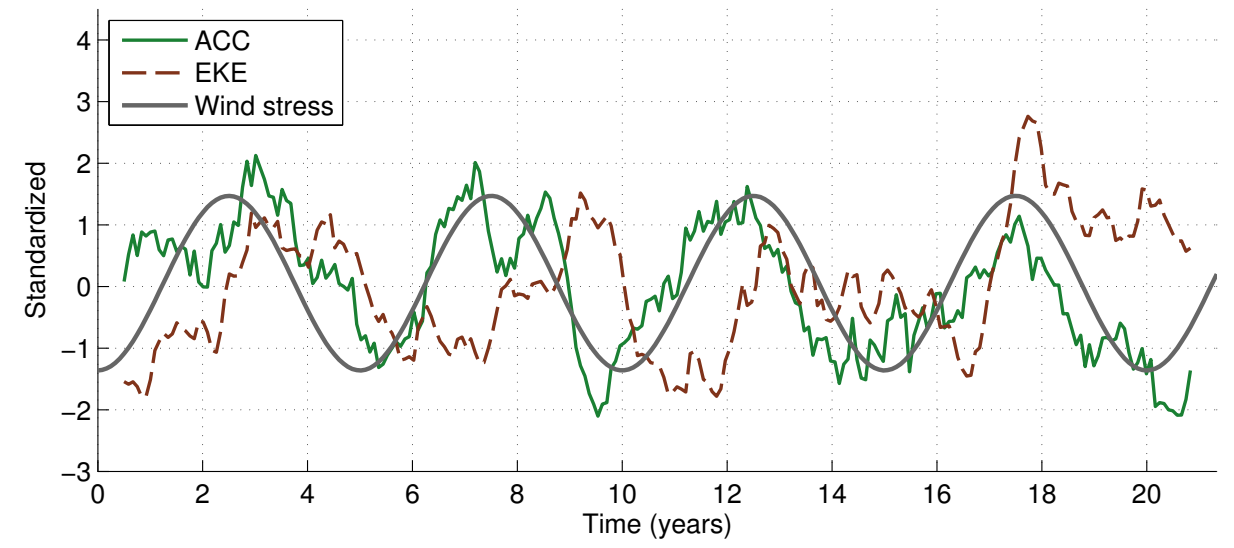

FIG. 6.3. Time series of standardized circumpolar transport (ACC) and eddy kinetic energy (EKE) shown with periodic wind stress forcing. Both the ACC and EKE time series have been passed through a 13-month running mean filter in order to smooth out the variability.

\subsubsection{Diffusivity}

Eddy diffusivity depends on both local eddy activity and on the temperature gradient. Here we diagnose the eddy diffusivity using the simple approach described in section 5.2.5. We evaluate the temperature gradient across the channel $T_{y}$, the eddy kinetic energy (EKE), and the diffusivity from $K_{T}=-\overline{v^{\prime} T^{\prime}} / \partial_{y} T$. Standardized time series of these quantities are shown in Figure 6.5. We see a decadal-scale decrease in the temperature gradient across the channel. Wind stress appears to drive the diffusivity, especially over the last two full cycles of the simulation. The maximum lag correlation between forcing and diffusivity occurs at 

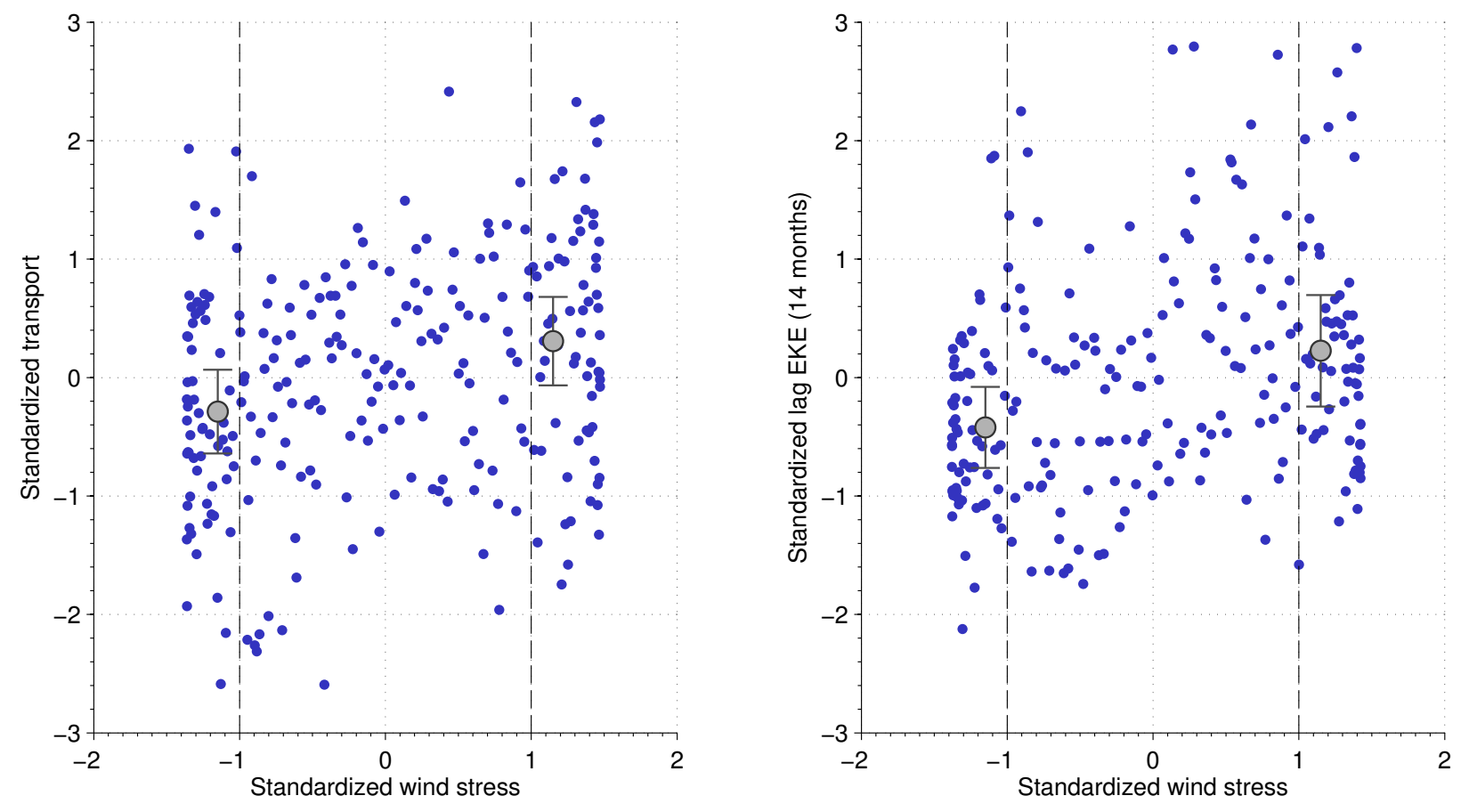

Fig. 6.4. Scatter plot of wind stress versus transport (left) and wind stress versus eddy kinetic energy (right). Composite means (gray circles) are shown with $95 \%$ confidence bounds. 
4 months, with a value of 0.6 if the full record is used and a value of 0.8 of only the last half of the data is selected. We attempt to diagnose the sensitivity of the diffusivity to wind stress by performing simple regression on the logarithmic four-month lag diffusivity $\log \left(K_{4}\right)$ as a function of the logarithm of the wind stress $\log (\tau)$. By fitting in the logarithm, we are essentially fitting $K_{4}(\tau)$ to a power law profile $y=x^{n}$, $\operatorname{such}$ that $\log (y)=n \log (x)$. The slope of the regression line provides an estimate for $n$. The decorrelation timescale of $K$ is about 3 months, giving us 83 independent measurements of $K$ when the lag is taken into account. We estimate $n=0.45 \pm 0.3(95 \% \mathrm{CI})$, so $0.15<n<0.75$. This range has some overlap with recent estimates from scaling theory that place $n$ between 0.5 and 1.5 (Meredith et al., 2012). In an averaged sense, our model displays slightly weaker compensation than that predicted by scaling theory. Increasing the horizontal resolution of the model may increase the degree of compensation. Assuming no change in scatter, increasing the number of degrees of freedom by a factor of 10 would decrease the margin of error for $n$ from 0.3 to about 0.1 .

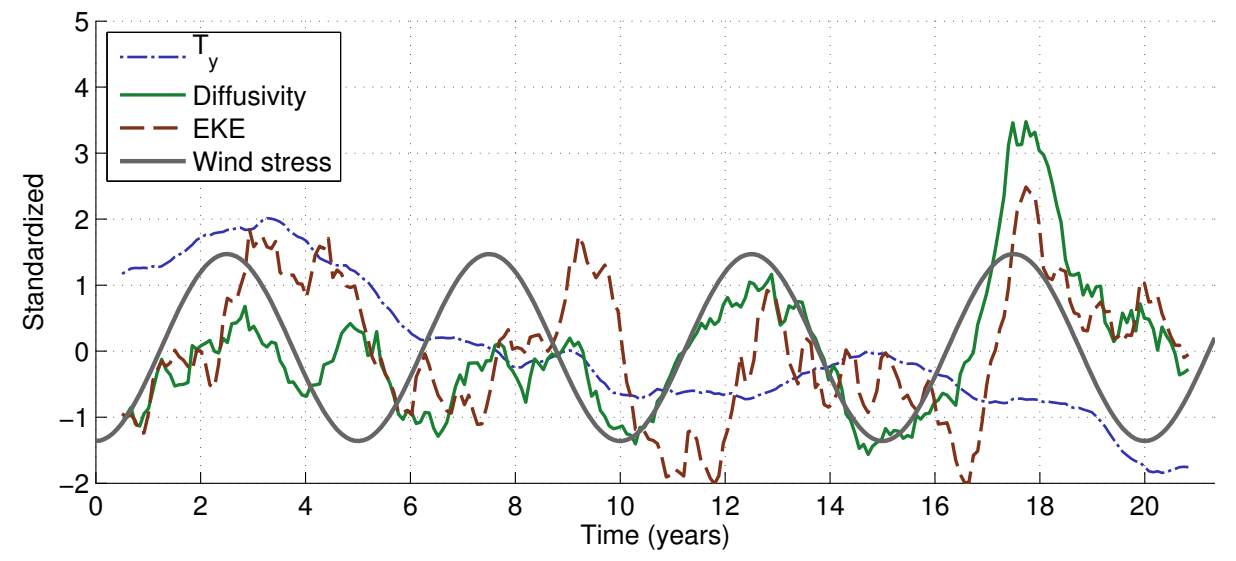

FIG. 6.5. Time series of standardized meridional temperature gradient across the channel $\left(T_{y}\right)$, mean eddy diffusivity in the channel, eddy kinetic energy (EKE), and periodic wind stress forcing. All fields except the wind stress have been passed through a 13-month smoothing filter in order to clarify the interannual variability. 


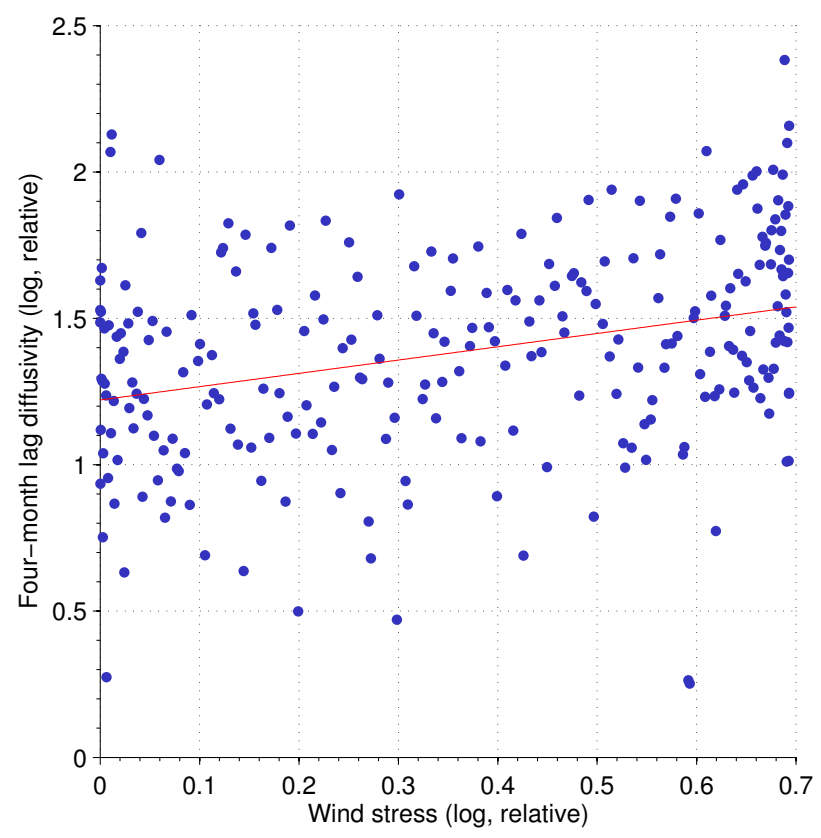

FIG. 6.6. Normalized, four-month lag diffusivity (logarithmic) versus normalized wind stress (logarithmic). The lag correlation between wind stress and diffusivity peaks at four months.

\subsubsection{Northward heat transport}

In Chapter 5 we found that mean and eddy-driven heat transport have roughly the same sensitivity to changes in wind stress, implying that eddy compensation (with heat transport used as a metric) is a good approximation on decadal timescales. Here we test the degree of compensation on interannual timescales. None of the net heat transport fields show an especially strong correlation $\left(r^{2}\right)$ with wind stress (mean 0.5, stationary 0.2 , transient 0.1 , and net 0.0). However, composite analysis suggests that weaker/stronger wind stress can shift the distribution of mean and eddy transport towards lower/higher magnitudes. The net heat transport shows no such composite difference, which indicates that eddy compensation (of heat transport) holds on interannual timescales. 


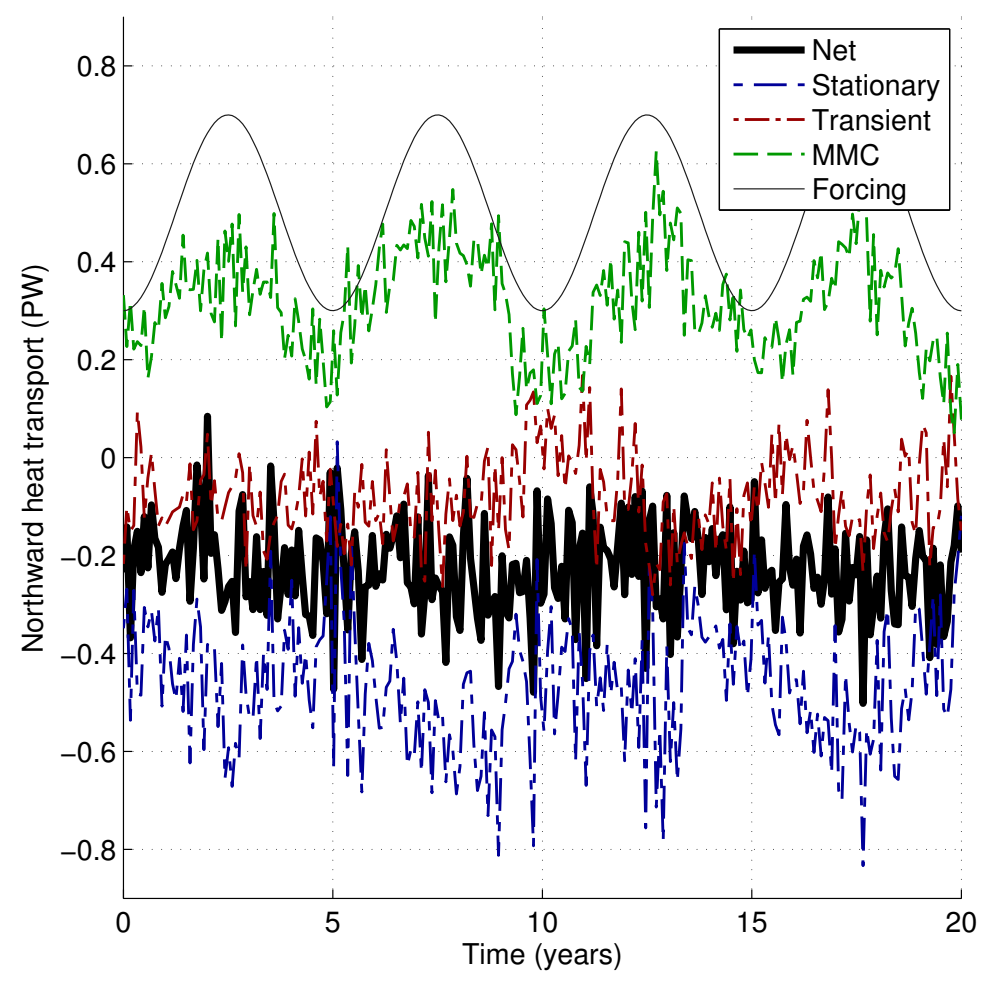

FIG. 6.7. Northward heat transport by component in the periodic forcing experiment

\subsubsection{Zonal mean jets}

The first two EOFs are similar to those found in the fine-resolution step response experiment, though they display increased power in the western boundary current. This is somewhat expected, as the EOFs illustrate a natural mode of variability (as opposed to a forced mode) set largely by the bathymetry. The PC time series of EOF1 and EOF2 are not correlated with wind stress $\left(r^{2}<0.1\right)$, which implies that the storm track positions are not set by interannual forcing variability (see Figure 6.8). However, mesoscale variability in the channel and gyre do increase wind wind stress, so we expect the intensity and frequency of oceanic "storms" that propagate along the length of the jet to increase, even if the jet positions stay roughly constant. 

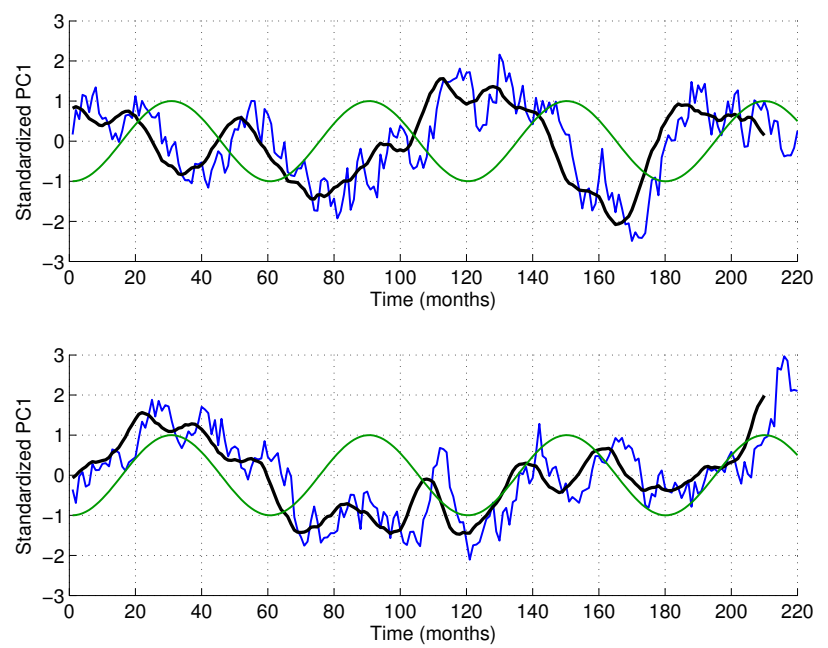

FIG. 6.8. Standardized principal component (PC) times series associated with the first two EOFs (thin blue line), a ten-year mean (thick black line), and the forcing (thin green line).

\subsection{MID-OCEAN SEAMOUNT}

The adjustment of the ocean to changing wind stress involves both transient and stationary eddies. As we saw in previous chapters, increasing wind stress can alter the spatial structure of circumpolar flow such that poleward heat transport by the stationary eddy component increases. Since bathymetry can constrain the mean position of the circumpolar current, it may be possible to reduce or eliminate the heat flux by stationary eddies in an idealized model by adding bathymetric features that intersect with the depth-integrated flow.

In this section, we perform a periodic forcing experiment using the time-dependent wind stress profile described in section 6.1 and sector model bathymetry with a meridionallyoriented seamount as described in section 4.21. The seamount forces contours of $f / h$ to converge in the mid-ocean and should constrain the flow to be less zonal. Figure 6.9 shows the annual mean barotropic streamfunction averaged during a 12-month period when the wind is near its minimum (left) and maximum (right). Surprisingly, the current meander appears to be consistently wider when the wind stress is weak. Stronger winds actually 
force the current to become more zonal, in contrast to what we saw in simulations with a continental ridge but a flat interior. The mesoscale jets also appear to be confined to the western half of the basin. The mean flow is strongly nonzonal and unstable upstream of the seamount; there it generates the characteristic westward propagating anomalies that eventually collect in the western boundary current (see section 5.4). The mean flow continues past the seamount, but instability seems to be suppressed in the eastern half of the basin. It may be that the current is too weak past the seamount for the nonzonal instability to dominate.
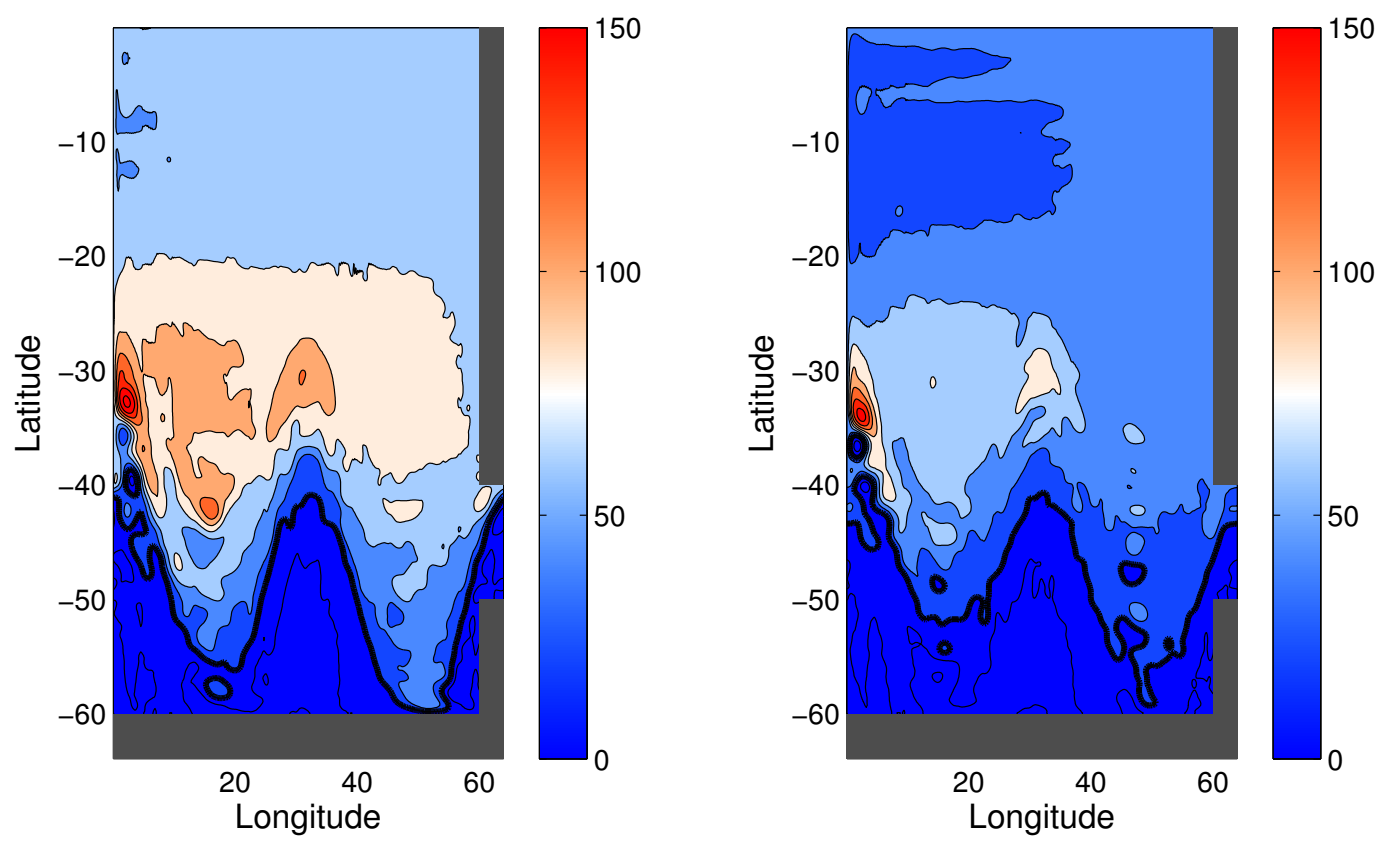

FIG. 6.9. Annual mean barotropic streamfunction for the one-bump case during a period when the wind is near its minimum value (left, months 174 to 186) and maximum value (right, months 84 to 96). The $20 \mathrm{~Sv}$ contour is plotted as a thick black line.

The response of the meridional overturning circulation is also surprising. The mean circulation is strongly influenced by the wind, but the sense of the circulation is reversed compared with results from Chapter 5 (see Figure 6.10). Specifically, while the correlation is not especially strong due to intense variability $\left(r^{2}=0.38\right)$, composite analysis suggests that weak/strong winds tend to bias the mean circulation towards higher/lower values. The 
interannual variability of the residual circulation is dominated by the mean circulation, so the residual is sensitive to wind stress as well. Though it is difficult to tell from the plot, composite analysis also shows that the eddy response does have some wind stress sensitivity, in the sense that stronger winds are associated with a weakened eddy response. This result is rather counterintuitive and suggests that topography can drastically alter the oceanic response to changing winds.

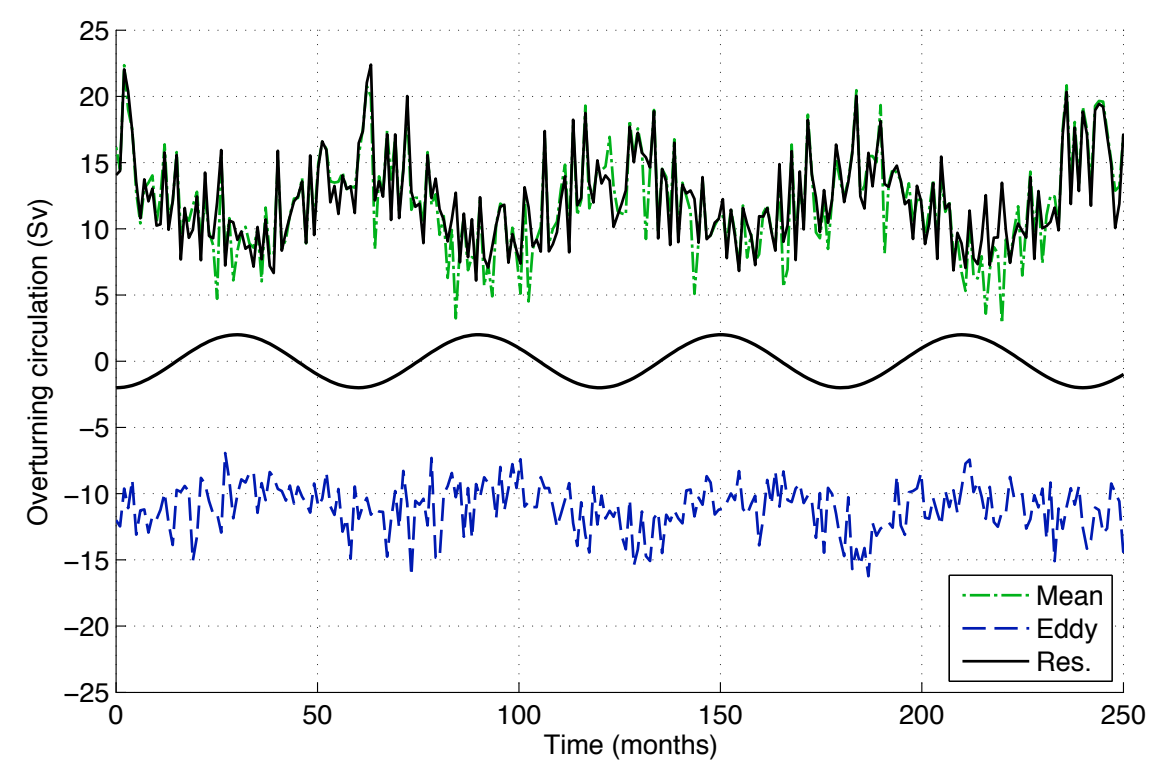

FIG. 6.10. Southern Ocean meridional overturning circulation by component. The smooth curve in the center represents the periodic component of wind stress forcing.

Finally, we examine the northward heat transport. As with the meridional overturning and barotropic streamfunction, stronger wind stress biases the mean heat transport towards lower values (see Figure 6.11). Stationary eddy heat transport also weakens during periods when the wind stress is low, such that changes between the mean and stationary transport cancel each other out. Since the transient eddy component is largely insensitive to changing wind stress and the other two components compensate, we see that the total, vertically-integrated heat transport is not sensitive to changing wind stress on interannual timescales. The results of this section are somewhat preliminary and suggest the need for further investigation of topographic effects. 


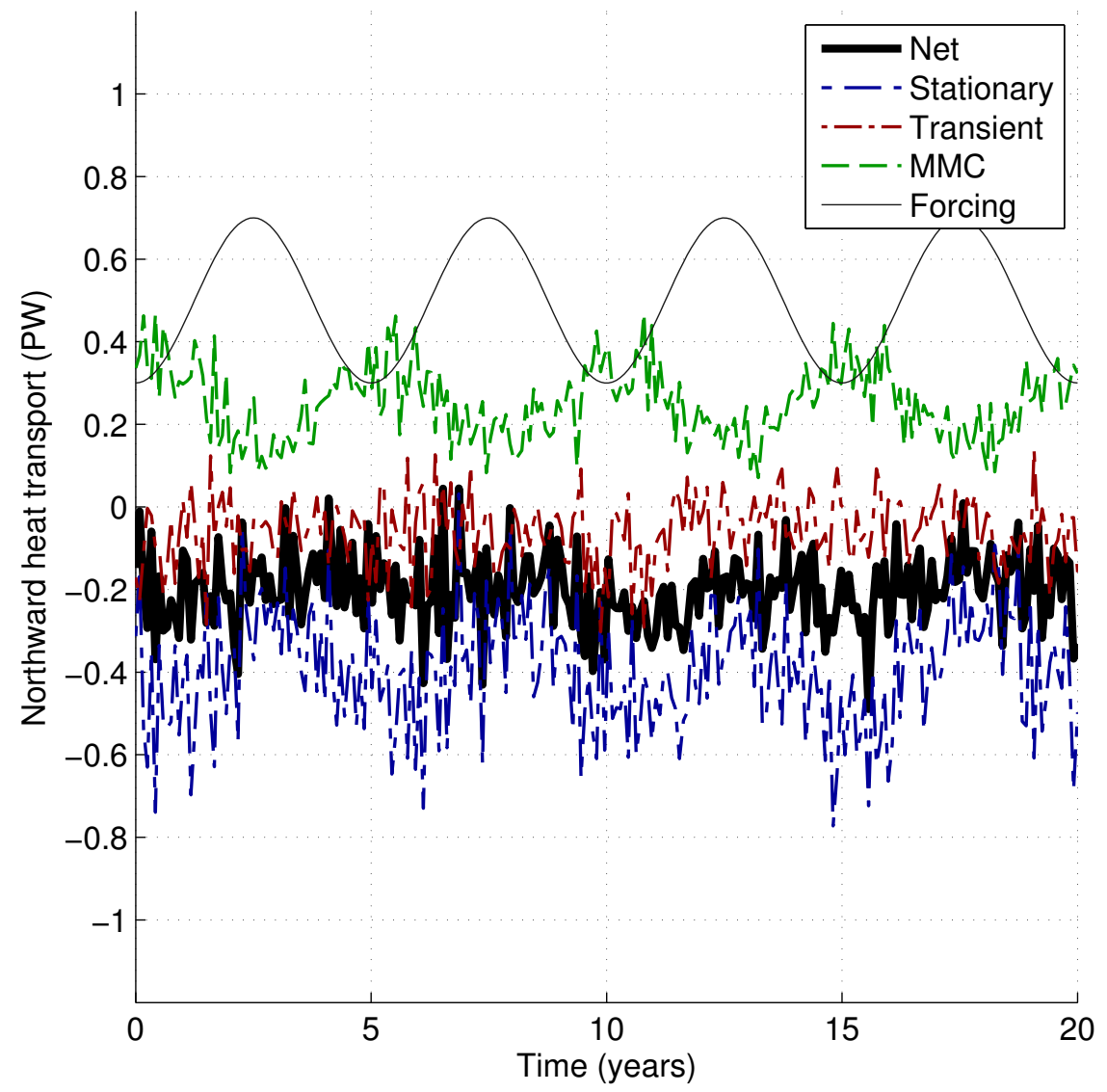

FIG. 6.11. Northward heat transport for the periodic forcing experiment with a mid-ocean ridge. 


\section{DISCUSSION AND CONCLUSIONS}

In this work we have attempted to better understand the sensitivity of the Southern Ocean to changes in wind stress using simple theories and idealized numerical simulations. Here we summarize our central results and discuss possible lines of future investigation.

\subsection{IS THE SOUTHERN OCEAN INSENSITIVE TO CHANGING WIND STRESS?}

The sector model is an idealized representation of an interhemispheric ocean basin with a circumpolar channel. It is capable of simulating both local Southern Ocean dynamics and global adjustment through the overturning circulation. In our experiments, the depthintegrated flow exhibits two dominant response patterns, illustrated in Figure 7.1. The two patterns are (i.) a change in the position of the circumpolar current that leads to stronger poleward eddy heat transport and (ii.) the intensification of the subtropical gyre. Pattern (i.) leads to subsurface warming in the subpolar region, and pattern (ii.) produces subsurface warming in the gyre region. Both warming signatures push isopycnal surfaces deeper into the interior, thereby altering SO stratification on decadal to centennial timescales. While care should be taken in comparing our highly idealized model with observations, we note that qualitatively similar subsurface heating trends have been observed in the synthesis of hydrographic and autonomous float data. Specifically, Roemmich et al. (2007) shows that the South Pacific subtropical gyre has spun up on a decadal timescale, likely in response to stronger Southern Hemispheric winds. High-latitude warming in the Southern Ocean has also been detected (Gille, 2008).

The transition region between circumpolar-current dominated latitudes and gyre-dominated latitudes is a dynamically interesting area. The mean isopycnal slope can change rapidly there, especially as seen in the doubled wind case (see Figure 7.2). The change in slope 


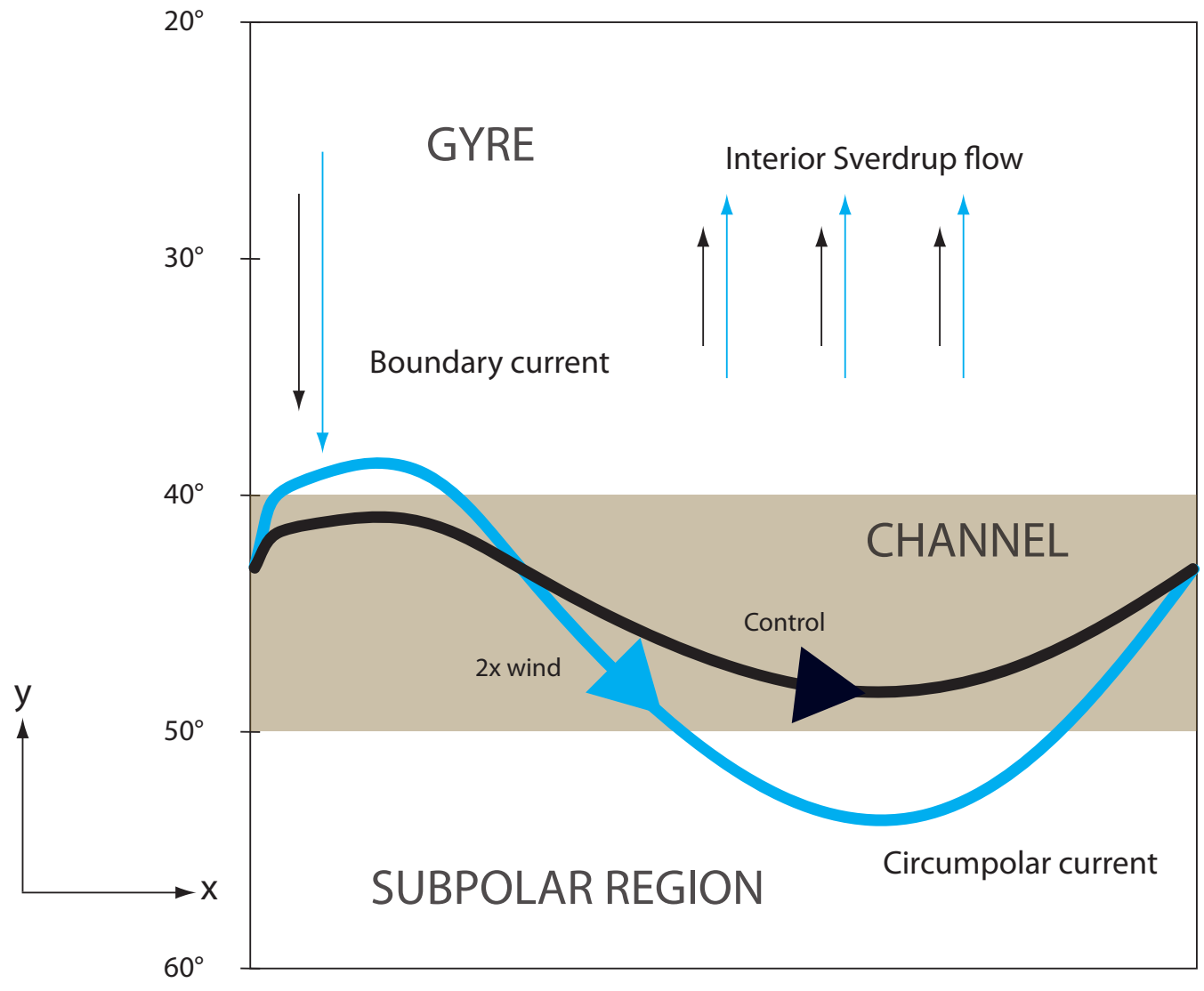

FIG. 7.1. The two dominant response patterns of depth-integrated flow to increased wind stress in the sector model. Flow associated with the control case is shown in black, and flow associated with the doubled wind case is shown in blue. The arrows in the gyre region are scaled as the strength of depth-integrated meridional flow. 
(if it exists) flips the sign of eddy heat flux from equatorward to poleward in the interior. The mean slope is set by a balance between direct wind-driven flow and eddy fluxes. Nevertheless, since the Eulerian mean and eddy induced overturning circulations do not have the same vertical structure (and since the gyre and channel regions respond differently to wind stress), a sustained increase in wind stress can eventually change the stratification of the SO.

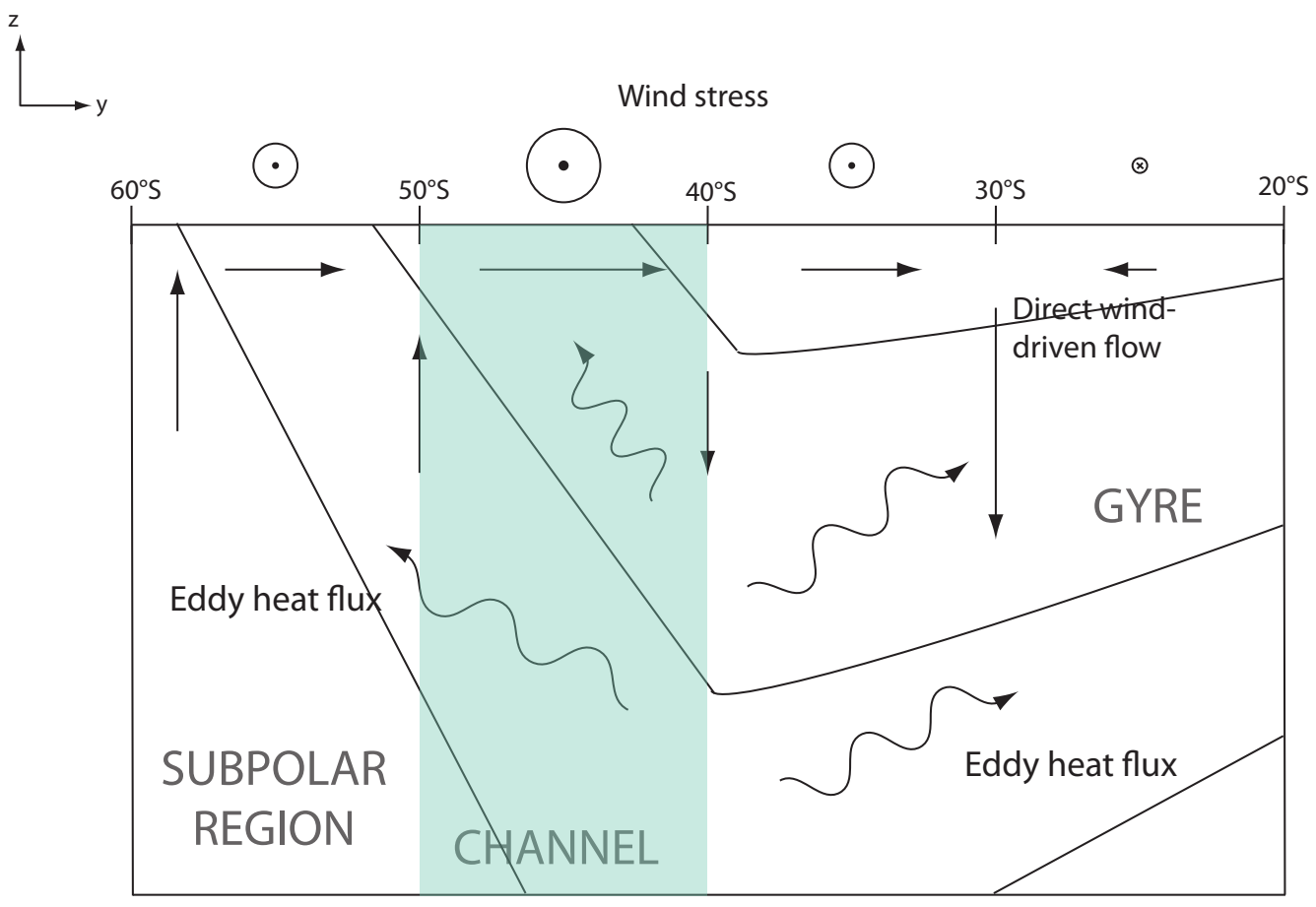

FIG. 7.2. Schematic of circulation and buoyancy in the doubled wind case showing the change in slope crossing from the channel to gyre region and the opposite sense of eddy heat flux. 
The SO wind-driven adjustment problem must be addressed across a range of temporal scales. On interannual timescales, strong winds can bias circumpolar transport and the meridional overturning circulation (as measured in depth-coordinate space) towards higher values, though the depth-integrated heat transport displays little to no sensitivity to changes in forcing. In the sector model, most of the heat transport compensation is accomplished by stationary eddies. On decadal to centennial timescales, the meander of the circumpolar current and the spin-up of the subtropical gyre change the density structure of the SO. The local deepening of the SO pycnocline changes the global distribution of fluxes between the surface and deep ocean, producing a centennial adjustment signal in the global pycnocline depth.

The response of the sector model is qualitatively consistent with the predictions of the simple global pycnocline model presented in Chapter 2. Stronger wind stress generally causes the ocean to take up more heat from the atmosphere and the deepens the global pycnocline. The equilibration of the SO with the rest of the ocean occurs on multi-decadal to centennial timescales, longer than the available observational record. Finally, the sensitivity of the global pycnocline to changing wind stress decreases as the nonlinearity of the eddies increases (assuming that explicitly resolved eddies are more "nonlinear" than their parameterized counterparts, where nonlinearity loosely refers to the sensitivity of the eddies to some local dynamical variable, such as the pycnocline depth). Despite its simplicity, the simple pycnocline model from Chapter 2 is useful for understanding the long-term adjustment processes.

Many of our results are inconsistent with a strict interpretation of the eddy saturation hypothesis. The additional energy imparted to the flow via stronger wind stress ends up in both the mean flow (as measured by the circumpolar transport) and in the eddy kinetic energy. However, note that the circumpolar transport increase is around $65 \%$ in the coarse resolution experiment and about $35 \%$ in the eddy-permitting runs. Decreasing the horizontal 
resolution below $1 / 6^{\circ}$ may further reduce (or practically eliminate) the sensitivity of the mean transport to wind stress. As such, simulations at higher resolution are needed.

Our results also suggest that the eddy compensation hypothesis is not an especially strong constraint. The residual circulation, eddy diffusivity, and isopycnal slope all display some sensitivity to wind stress on interannual, decadal, and centennial timescales. The adjustment process is rate-limited by communication between the Southern Ocean and the global ocean through the pycnocline, so it may take many centuries to millennia for the SO to

full equilibrate to a sustained increase in wind stress. The long-term adjustment mechanism described here may be relevant when studying glacial cycles or other processes on geologic timescales. It may also be relevant for global climate. If the overturning circulation is likely to respond to a sustained increase in wind stress, then the strength of the SO carbon sink will likely be altered on multi-decadal timescales. Our results are consistent with those of Meredith et al. (2012), who show using scaling arguments and numerical simulations that the ocean's overturning circulation will likely change substantially in the coming decades and centuries under decadally varying wind stress and eddy fields. More realistic, centennial-scale simulations and continued monitoring of the Southern Ocean density structure are needed in order to better quantify the sensitivity of SO mean circulation and eddy fields to changing wind stress.

\subsection{MODELING APPROACH}

In an effort to obtain simpler (but still dynamically relevant) results, we neglected many sources of complexity that are present in the real ocean. Our results are robust within our set of experiments, but our model behavior should be compared with more realistic experiments in a model hierarchy in order to determine the effect of factors such as sea ice, salinity, surface freshwater fluxes, realistic bathymetry, icebergs, and mesoscale variability on the wind-driven adjustment problem. 


\subsubsection{Eddy parameterization}

Global scale, eddy-permitting climate models remain computationally expensive. They only become more so as new fields and sets of equations are added (e.g. biogeochemistry, sea ice, salinity). As such, running a modern climate model on the scale of multiple centuries to millennia remains a major undertaking. Such long-term runs must parameterize the effect of eddies on the mean buoyancy and momentum distributions. Unfortunately, experience with many numerical simulations (including the ones described here) suggests that current eddy parameterization schemes do not correctly represent the mean effect of eddies on the mean flow, especially not on decadal or longer timescales.

A possible solution, though a bit of an ad-hoc one, is to allow the sensitivity of the eddy bolus velocity to isopycnal slope to be of higher order, following the generalized $n$ approach discussed in Chapter 2:

$$
\mathbf{K}_{\mathbf{T}}=\kappa_{a}\left[\begin{array}{ccc}
0 & 0 & -s_{x}^{n} \\
0 & 0 & -s_{y}^{n} \\
s_{x}^{n} & s_{y}^{n} & 0
\end{array}\right],
$$

where $n$ is a dimensionless index that allows us to adjust the sensitivity of eddy transport to the isopycnal slope. The case $n=1$ roughly corresponds to the Gent-McWilliams scheme (Gent and Mcwilliams, 1990), and the case $n=2$ roughly corresponds to the extension proposed by Visbeck et al. (1997). The value of $n$ and its possible functional dependence on wind stress $n=n(\tau)$ could be constrained by sets of eddy-permitting runs. In order to implement the above parameterization of $K_{T}$, we need to define the appropriate scale for the isopycnal slope $s$. Specifically, we need to understand the typical lengths over which the eddy diffusivity affects and is affected by the large-scale structure. This is somewhat analogous to the problem of setting vertical mixing lengths in a scheme like KPP. The above scheme (equation 7.2.1) allows for the possibility of eddy saturation at high values of $n$. Another approach to parameterization is to solve a prognostic equation for eddy kinetic energy (EKE) 
and use it to derive the isopycnal eddy diffusivity (Eden and Greatbatch, 2008). This approach is grounded in the observed increase in EKE with wind stress (Abernathey et al., 2011) and also exhibits the potential for saturation behavior.

\subsubsection{Variable resolution}

The sector model is useful for studying interhemispheric adjustment problems such as the one discussed in this work. Unfortunately, it is computationally expensive to run at eddy permitting resolution in its current configuration, since the computational grid is uniform in longitude-latitude. The model spends a significant amount of processing power explicitly simulating the northern hemisphere, which affords the Southern Ocean a fairly realistic northern boundary condition (i.e. dynamic stratification that can feed back on local changes in SO density structure) but arguably wastes processing power. It may be possible to reduce the horizontal resolution in the northern hemisphere, sacrificing some degree of accuracy in favor of computational speed. Ideally, this would be accomplished by a discretization scheme where the horizontal resolution changes slowly with latitude in order to discourage the formation of computational modes where the grid changes size. For example, we may run the Southern Ocean at $1 / 6^{\circ}$ in the horizontal and smoothly transition to $2^{\circ}$ in the Northern

Hemisphere. For studies of sub-decadal scale circulation, it is likely sufficient to restore the stratification to a given profile at the northern boundary, since interhemispheric adjustment is of decadal order and above.

\subsection{FUTURE WORK}

The results presented here are by no means conclusive. Here we briefly discuss some possible lines of future investigation. 


\subsubsection{Automatic differentiation}

In this work we performed basic sensitivity analysis using pairs of control/experimental simulations. An alternative approach is to use automatic differentiation, which involves automatically generating code to calculate partial derivatives from a fully nonlinear prognostic code (Adcroft et al., 2010). This can be accomplished using an adjoint model compiler, for example. We can potentially use this approach to generate spatial maps of the sensitivity of slope, diffusivity, and the residual circulation to changing wind stress (e.g. $\partial K / \partial \tau$, linearized about the basic state of the model).

\subsubsection{Latitude of wind forcing}

The recent decadal trend in the SAM indicates both a spin-up and a poleward shift of westerly winds. In this work we only changed the magnitude of the winds but not its position. It could be instructive to perform step response and periodic forcing experiments where the variable quantity is the latitude of maximum wind stress forcing. Nevertheless, we found that it is possible to change the path of the circumpolar current by simply changing the magnitude of the wind stress and not its position, at least in those regions where the flow is not strongly constrained by the bathymetry. As such, the path of the circumpolar current is complex and set by a variety of factors.

\subsubsection{Bathymetry and surface forcing}

The sector model is highly idealized, which is useful for the kind of study presented here. However, it may be instructive to further develop the hierarchy of models by adding several sources of complexity. For example, we might run a (still idealized) version of the sector model with more than one interhemispheric basin to study the inter-basin adjustment problem. Going a few steps beyond that, we could use fully realistic bathymetry. Results 
from section 6.2 suggest that the structure of the ocean basin can drastically alter the sensitivity of the circulation to changing wind stress, so a detailed study of bathymetric effects (including a fully realistic case) will be crucial for understanding the future evolution of the carbon sink. It will also be important to employ more realistic wind and buoyancy flux fields in order to "bridge the gap" between idealized studies and more realistic simulations.

\subsubsection{Cryosphere}

A significant fraction of the Southern Ocean is covered with seasonally varying sea ice. High sea ice coverage raises albedo, suppresses oceanic convection, and hinders the flux of heat across the ocean surface. Though sea ice and its variability likely have a significant

impact on SO dynamics, this component of the Southern Ocean system is often neglected in basic theories. Calving icebergs provide a freshwater flux into the Southern Ocean and may also have some regional-scale dynamical impact. Incorporating sea ice and icebergs into basic dynamical frameworks is an important goal and should be an interesting area of study in the future.

\subsubsection{Mesoscale jets}

In Chapter 5, we briefly discussed the existence of alternating quasi-zonal jets in the gyre and channel regions of the sector model. Quasi-zonal jets have been directly measured in the ocean (Hogg and Brechnerowens (1999), van Sebille et al. (2011)), detected in satellite altimetry data (Maximenko et al., 2008), and simulated in numerical models (Nakano and Hasumi, 2005). Recently, Wang et al. (2012) explored the possibility that such quasi-zonal jets are formed at the eastern boundary of basins via the instability of eastern boundary currents. In the future, we wish to better understand the dynamics of the zonal jets that occur in the eddy permitting sector model. This can be accomplished in part by performing careful scale analysis (to see if such features are indeed robustly consistent with the Rhines 
scale), examining the energy spectrum of the jets, and comparing numerical simulation results with simple models that include wind forcing, bathymetry, and turbulent dissipation (Nadiga, 2006).

\subsubsection{Buoyancy forcing}

Much of the available literature on SO sensitivity deals with changes in the wind stress. However, numerical studies suggest that the overturning circulation is likely very sensitive to buoyancy and freshwater forcing as well, especially on glacial timescales (Morrison et al., 2011). The step response approach may be a profitable one in this area. For example, we could start with the control profile discussed in section 3.3 and instantaneously switch to a warmer profile constructed by adding the control profile to a linear function of latitude. Projections from recent numerical simulations suggest an appropriate choice for the endpoints of the linear perturbation: $2^{\circ}$ at the southern end of the sector domain and $4^{\circ}$ at the northern edge, reflecting a difference in surface temperatures comparing 1980-1999 and 2080-2099. The additional flux-driven warming should deepen the pycnocline and eventually cause globalscale changes in flux between the upper and lower ocean.

\subsubsection{Carbon cycle}

We hope in the near future to address the evolution of the Southern Ocean carbon sink in the coming decades and centuries. If the overturning circulation strengthens and thereby increases the upwelling of DIC-rich waters into the surface ocean, the effectiveness of the carbon sink may be reduced. However, the biogeochemistry of the Southern Ocean provides a dynamic set of feedbacks that complicate our efforts to make such simple predictions. The spatial pattern of air-sea carbon flux is also complex. Preliminary results from a sector model

run with a simple biogeochemistry model suggest that the Southern Ocean sink for natural $\mathrm{CO}_{2}$ may actually increase under stronger wind stress (Lovenduski et al., 2011). This can 
occur, for example, if the increase in high-latitude outgassing is overwhelmed by an increase in mid-latitude uptake. High-resolution modeling of biogeochemical cycling will remain a fruitful area of investigation for the foreseeable future. 


\section{REFERENCES}

Abernathey, R., J. Marshall, and D. Ferreira, 2011: The dependence of Southern Ocean meridional overturning on wind stress. Journal of Physical Oceanography, 41 (12), 22612278, doi:10.1175/JPO-D-11-023.1.

Adcroft, A., et al., 2010: MITgcm user manual. mitgcm.org.

Allison, L., H. Johnson, and D. P. Marshall, 2011: Spin-up and adjustment of the Antarctic Circumpolar Current and global pycnocline. Journal of Marine Research, 69 (2-3), 167189.

Andrews, D. and M. McIntyre, 1976: Planetary waves in horizontal and vertical shear: The generalized Eliassen-Palm relation and the mean zonal acceleration. Journal of the Atmospheric Sciences, 33 (11), 2031-2048.

Baker-Yeboah, S., D. R. Watts, and D. A. Byrne, 2009: Measurements of sea surface height variability in the Eastern South Atlantic from pressure sensor-equipped inverted echo sounders: Baroclinic and barotropic components. J. Atmos. Oceanic Technol., 26 (12), 2593-2609, doi:10.1175/2009JTECHO659.1.

Böning, C., A. Dispert, M. Visbeck, and S. Rintoul, 2008: The response of the Antarctic Circumpolar Current to recent climate change. Nature Geoscience, 1, 864-869.

Cessi, P. and P. Otheguy, 2003: Oceanic teleconnections: Remote response to decadal wind forcing. Journal of Physical Oceanography, 33 (8), 1604-1617.

Chelton, D. B. and M. G. Schlax, 1996: Global observations of oceanic Rossby waves. Science, 272, 234-238.

Dee, D. and S. Uppala, 2009: Variational bias correction of satellite radiance data in the ERA interim reanalysis. Quarterly Journal of the Royal Meteorological Society, 135, 1830-1841.

Eden, C. and R. Greatbatch, 2008: Towards a mesoscale eddy closure. Ocean Modelling, 20, $223-239$.

Farneti, R., T. L. Delworth, A. J. Rosati, S. M. Griffies, and F. Zeng, 2010: The role of mesoscale eddies in the rectification of the Southern Ocean response to climate change. Journal of Physical Oceanography, 40 (7), 1539-1557, doi:10.1175/2010JPO4353.1.

Ferrari, R. and M. Nikurashin, 2010: Suppression of eddy diffusivity across jets in the Southern Ocean. Journal of Physical Oceanography, 40, 1501-1519, doi:10.1175/2010JPO4278.1.

Fyfe, J. and O. Saenko, 2005: Human-induced change in the Antarctic Circumpolar Current. J Climate, 18, 3068-3073. 
Gent, P., 2011: The Gent-McWilliams parameterization: 20/20 hindsight. Ocean Modelling, 39 (1), 2-9.

Gent, P. and J. Mcwilliams, 1990: Isopycnal mixing in ocean circulation models. Journal of Physical Oceanography, 20, 150-155.

Gille, S., 2002: Warming of the Southern Ocean since the 1950s. Science, 295, 1275-1277, doi:10.1126/science.1065863.

Gille, S., 2003: Float observations of the Southern Ocean. part i: Estimating mean fields, bottom velocities, and topographic steering. Journal of Physical Oceanography, 33 (6), 1167-1181.

Gille, S., 2008: Decadal-scale temperature trends in the Southern Hemisphere ocean. $J$ Climate, 21, 4749-4765, doi:10.1175/2008JCLI2131.1.

Gille, S., E. Metzger, and R. Tokmakian, 2004: Seafloor topography and ocean circulation. Oceanography, 17 (1), 47-54.

Gille, S., D. Stevens, R. Tokmakian, and K. Heywood, 2001: Antarctic Circumpolar Current response to zonally averaged winds. Journal of Geophysical Research, 106 (C2), 27432759 .

Gnanadesikan, A., 1999: A simple predictive model for the structure of the oceanic pycnocline. Science, 283, 2077-2079.

Gordon, A., E. Molinelli, and T. Baker, 1978: Large-scale relative dynamic topography of the Southern Ocean. Journal of Geophysical Research, 83 (C6), 3023-3032.

Griffies, S., 1998: The Gent-McWilliams skew flux. Journal of Physical Oceanography, 28, 831-841.

Hallberg, R. and A. Gnanadesikan, 2001: An exploration of the role of transient eddies in determining the transport of a zonally reentrant current. Journal of Physical Oceanography, 31, 3312-3330.

Hallberg, R. and A. Gnanadesikan, 2006: The role of eddies in determining the structure and response of the wind-driven Southern Hemisphere overturning: Results from the Modeling Eddies in the Southern Ocean (MESO) project. Journal of Physical Oceanography, 36, $2232-2252$.

Harris, J. W. and H. Stocker, 1998: Handbook of mathematics and computational science. Springer.

Held, I., 2005: The gap between simulation and understanding in climate modeling. Bulletin of the American Meteorological Society, 86 (11), 1609-1614.

Hogg, A. and J. Blundell, 2006: Interdecadal variability of the Southern Ocean. Journal of Physical Oceanography, 36, 1626-1645. 
Hogg, A. and W. Brechnerowens, 1999: Direct measurement of the deep circulation within the Brazil Basin. Deep Sea Research Part II: Topical Studies in Oceanography, 46, 335353, doi:10.1016/S0967-0645(98)00097-6.

Hovmöller, E., 1949: The trough and ridge diagram. Tellus, 1 (2), 62-66.

Ito, T. and J. Marshall, 2008: Control of lower-limb overturning circulation in the Southern Ocean by diapycnal mixing and mesoscale eddy transfer. Journal of Physical Oceanography, 38, 2832-2845.

Ito, T., M. Woloszyn, and M. Mazloff, 2010: Anthropogenic carbon dioxide transport in the Southern Ocean driven by Ekman flow. Nature, 463, 80, doi:10.1038/nature08687.

Jones, D. C., T. Ito, and N. S. Lovenduski, 2011: The transient response of the Southern Ocean pycnocline to changing atmospheric winds. Geophysical Research Letters, 38 (15), L15 604, doi:10.1029/2011GL048145.

Kalnay, E., et al., 1996: The NCEP/NCAR 40-year reanalysis project. Bulletin of the American Meteorological Society, 77 (3), 437-471.

Kang, Y., J. Price, and L. Magaard, 1982: On stable and unstable Rossby waves in non-zonal oceanic shear flow. Journal of Physical Oceanography, 12 (6), 528-537.

Karsten, R., H. Jones, and J. Marshall, 2002: The role of eddy transfer in setting the stratification and transport of a circumpolar current. Journal of Physical Oceanography, $32,39-54$.

Karsten, R. and J. Marshall, 2002: Testing theories of the vertical stratification of the ACC against observations. Dynamics of Atmospheres and Oceans, 36, 233-246.

Killworth, P., 1992: An equivalent-barotropic mode in the Fine Resolution Antarctic Model. Journal of Physical Oceanography, 22 (11), 1379-1387.

Köhl, A., D. Dommenget, K. Ueyoshi, and D. Stammer, 2007: The Global ECCO 1952 to 2001 ocean synthesis. National Oceanogr. Partnership Program, Washington, D. C.

Krupitsky, A., V. Kamenkovich, N. Naik, and M. Cane, 1996: A linear equivalent barotropic model of the Antarctic Circumpolar Current with realistic coastlines and bottom topography. Journal of Physical Oceanography, 26 (9), 1803-1824.

LaCasce, J., 2000: Floats and f/H. Journal of Marine Research, 58, 61-95.

Large, W., J. McWilliams, and S. Doney, 1994: Oceanic vertical mixing: A review and a model with a nonlocal boundary layer parameterization. Reviews of Geophysics, 32, 363-403.

Large, W. and S. Yeager, 2009: The global climatology of an interannually varying air-sea flux data set. Climate Dynamics, 33, 341-364, doi:10.1007/s00382-008-0441-3. 
LeQuéré, C., et al., 2007: Saturation of the Southern Ocean CO2 sink due to recent climate change. Science, 316 (5832), 1735.

Lorenz, E., 1956: Empirical orthogonal functions and statistical weather prediction. Scientific Report No. 1, Statistical Forecasting Project, Air Force Research Laboratories, Office of Aerospace Research, USAF, Bedford, MA.

Lovenduski, N. and T. Ito, 2009: The future evolution of the Southern Ocean CO2 sink. Journal of Marine Research, 67, 597-617.

Lovenduski, N., T. Ito, and D. Jones, 2011: The impact of increased wind stress on the Southern Ocean carbon distribution: Results from a high-resolution ocean model. World Climate Research Programme Open Science Conference, Denver, CO.

Lovenduski, N. S., N. Gruber, and S. C. Doney, 2008: Toward a mechanistic understanding of the decadal trends in the Southern Ocean carbon sink. Global Biogeochem. Cycles, 22 (3), GB3016, doi:10.1029/2007GB003139.

Marshall, D., 1995a: Influence of topography on the large-scale ocean circulation. Journal of Physical Oceanography, 25 (7), 1622-1635.

Marshall, D., 1995b: Topographic steering of the Antarctic Circumpolar Current. Journal of Physical Oceanography, 25 (7), 1636-1650.

Marshall, G., 2003: Trends in the Southern Annular Mode from observations and reanalyses. J Climate, 16, 4134-4143.

Marshall, J., A. Adcroft, C. Hill, and L. Perelman, 1997a: A finite-volume, incompressible Navier Stokes model for studies of the ocean on parallel computers. Journal of Geophysical Research, 102 (C3), 5753-5766.

Marshall, J., C. Hill, L. Perelman, and A. Adcroft, 1997b: Hydrostatic, quasi-hydrostatic, and nonhydrostatic ocean modeling. Journal of Geophysical Research, 102 (C3), 57335752 .

Marshall, J., H. Jones, R. Karsten, and R. Wardle, 2001: Can eddies set ocean stratification? Journal of Physical Oceanography, 32, 26-38.

Marshall, J. and T. Radko, 2003: Residual-mean solutions for the Antarctic Circumpolar Current and its associated overturning circulation. Journal of Physical Oceanography, 33, $2341-2354$.

Marshall, J. and K. Speer, 2012: Closure of the meridional overturning circulation through Southern Ocean upwelling. Nature Geoscience, 5 (3), 171-180, doi:10.1038/ngeo1391.

Matthews, A. and M. Meredith, 2004: Variability of Antarctic Circumpolar transport and the Southern annular mode associated with the madden-julian oscillation. Geophysical Research Letters, 31 (L24312), doi:10.1029/2004GL021666. 
Maximenko, N., O. Melnichenko, P. Niiler, and H. Sasaki, 2008: Stationary mesoscale jet-like features in the ocean. Geophysical Research Letters, 35, 8603, doi:10.1029/2008GL033267.

Mayewski, P., et al., 2009: State of the Antarctic and Southern Ocean climate system. Reviews of Geophysics, 47.

Meredith, M. and A. Hogg, 2006: Circumpolar response of Southern Ocean eddy activity to a change in the Southern Annular Mode. Geophysical Research Letters, 33 (16), L16 608.

Meredith, M. P., A. C. N. Garabato, A. M. Hogg, and R. Farneti, 2012: Sensitivity of the overturning circulation in the Southern Ocean to decadal changes in wind forcing. $J$ Climate, 25 (1), 99-110, doi:10.1175/2011JCLI4204.1.

Metzl, N., 2009: Decadal increase of oceanic carbon dioxide in Southern Indian Ocean surface waters (1991-2007). Deep Sea Research Part II, 56, 607-619.

Miller, R. L., G. A. Schmidt, and D. T. Shindell, 2006: Forced annular variations in the 20th century Intergovernmental Panel on Climate Change Fourth Assessment Report models. Journal of Geophysical Research, 111 (D18), D18 101, doi:doi:10.1029/2005JD006323.

Moore, J. K., M. R. Abbott, and J. G. Richman, 1999: Location and dynamics of the Antarctic Polar Front from satellite sea surface temperature data. Journal of Geophysical Research, 104 (C2), 3059-3073.

Morrison, A., A. Hogg, and M. L. Ward, 2011: Sensitivity of the Southern Ocean overturning circulation to surface buoyancy forcing. Geophysical Research Letters, 38 (L14602), doi: 10.1029/2011GL048031.

Munk, W. and E. Palmén, 1951: Note on the dynamics of the Antarctic Circumpolar Current. Tellus, 3 (1), 53-55.

Nadiga, B., 2006: On zonal jets in oceans. Geophysical Research Letters, 33, L10 601, doi: 10.1029/2006GL025865.

Nakano, H. and H. Hasumi, 2005: A series of zonal jets embedded in the broad zonal flows in the Pacific obtained in eddy-permitting ocean general circulation models. Journal of Physical Oceanography, 35, 474, doi:10.1175/JPO2698.1.

Nauw, J. and H. Dijkstra, 2001: The origin of low-frequency variability of double-gyre winddriven flows. Journal of Marine Research, 59, 567-597.

Onogi, K., J. Tsutsui, and H. Koide, 2007: The JRA-25 reanalysis. Journal of the Meteorological Society of Japan, 85 (3), 369-432.

Orsi, A., T. Whitworth, and W. Nowlin, 1995: On the meridional extent and fronts of the Antarctic Circumpolar Current. Deep Sea Research Part I, 42 (5), 641-673.

Rhines, P., 1975: Waves and turbulence on a beta-plane. Journal of Fluid Mechanics, 69 (03), 417-443. 
Rintoul, S., C. Hughes, and D. Olbers, 2001: The Antarctic Circumpolar Current system. International geophysics series, 271-302.

Roemmich, D., J. Gilson, and R. Davis, 2007: Decadal spinup of the South Pacific subtropical gyre. Journal of Physical Oceanography, 37, 162-173.

Russell, J., K. Dixon, A. Gnanadesikan, R. Stouffer, and J. Toggweiler, 2006: The Southern Hemisphere westerlies in a warming world: Propping open the door to the deep ocean. $J$ Climate, 19, 6382-6390.

Sabine, C., R. Feely, N. Gruber, R. Key, and K. Lee, 2004: The oceanic sink for anthropogenic CO2. Science, 305 (367), 367-371, doi:10.1126/science.1097403.

Salmon, R., 1998: Lectures on geophysical fluid dynamics. Oxford University Press.

Sarmiento, J., R. Slater, R. Barber, and L. Bopp, 2004: Response of ocean ecosystems to climate warming. Global Biogeochem. Cycles, 18 (GB3003), doi:10.1029/2003GB002134.

Sokolov, S. and S. Rintoul, 2009: Circumpolar structure and distribution of the Antarctic Circumpolar Current fronts: 1. mean circumpolar paths. Journal of Geophysical Research, 114 (C11018), doi:10.1029/2008JC005108.

Spall, M., 2000: Generation of strong mesoscale eddies by weak ocean gyres. Journal of Marine Research, 58, 97-116.

Speer, K., S. Rintoul, and B. Sloyan, 2000: The diabatic Deacon cell. Journal of Physical Oceanography, 30 (12), 3212-3222.

Straub, D., 1993: On the transport and angular momentum balance of channel models of the Antarctic Circumpolar Current. Journal of Physical Oceanography, 23, 776-782.

Thompson, A., 2008: The atmospheric ocean: eddies and jets in the Antarctic Circumpolar Current. Philosophical Transactions of the Royal Society A, 366, 4529-4541, doi:10.1098/ rsta.2008.0196.

Thompson, A., P. Haynes, and C. Wilson, 2010: Rapid Southern Ocean front transitions in an eddy-resolving ocean GCM. Geophysical Research Letters, 37, L23 602, doi:10.1029/ 2010 GL045386.

Thompson, A. and K. J. Richards, 2011: Low frequency variability of Southern Ocean jets. Journal of Geophysical Research, 116 (C9), C09 022, doi:10.1029/2010JC006749.

Thompson, D. W. J. and S. Solomon, 2002: Interpretation of recent Southern Hemisphere climate change. Science, 296 (296), 895-899, doi:10.1126/science.1069270.

Toggweiler, J. and B. Samuels, 1995: Effect of Drake Passage on the global thermohaline circulation. Deep Sea Research Part I, 42 (4), 477-500.

Treguier, A. M., J. L. Sommer, J. M. Molines, and B. D. Cuevas, 2010: Response of the Southern Ocean to the Southern Annular Mode: Interannual variability and multidecadal trend. Journal of Physical Oceanography, 40 (7), 1659-1668, doi:10.1175/2010JPO4364.1. 
Uppala, S., P. Kållberg, and A. Simmons, 2005: The ERA40 reanalysis. Quarterly Journal of the Royal Meteorological Society, 131 (612), 2961-3012, doi:10.1256/qj.04.176.

Vallis, G., 2006: Atmospheric and oceanic fluid dynamics: fundamentals and large-scale circulation. Cambridge University Press.

van Sebille, E., I. Kamenkovich, and J. K. Willis, 2011: Quasi-zonal jets in 3-D Argo data of the northeast Atlantic. Geophysical Research Letters, 38, 2606, doi:10.1029/ 2010GL046267.

Visbeck, M., J. Marshall, and T. Haine, 1997: Specification of eddy transfer coefficients in coarse-resolution ocean circulation models. Journal of Physical Oceanography, 27, 381402.

Völker, C., 1999: Momentum balance in zonal flows and resonance of baroclinic Rossby waves. Journal of Physical Oceanography, 29, 1666-1681.

Wang, J., M. A. Spall, G. R. Flierl, and P. Malanotte-Rizzoli, 2012: A new mechanism for the generation of quasi-zonal jets in the ocean. Geophysical Research Letters, 39 (10), L10 601, doi:10.1029/2012GL051861.

White, W. and R. Peterson, 1996: An Antarctic circumpolar wave in surface pressure, wind, temperature and sea-ice extent. Nature, 380 (6576), 699-702.

Wolff, J., E. Maier-Reimer, and D. Olbers, 1991: Wind-driven flow over topography in a zonal -plane channel: A quasi-geostrophic model of the Antarctic Circumpolar Current. Journal of Physical Oceanography, 21 (2), 236-264.

Wunsch, C., 1998: The work done by the wind on the oceanic general circulation. Journal of Physical Oceanography, 28, 2332-2340.

Zhang, B. and J. M. Klinck, 2008: The effect of Antarctic Circumpolar Current transport on the frontal variability in Drake Passage. Dynamics of Atmospheres and Oceans, 45 (3-4, SI), 208-228, doi:10.1016/j.dynatmoce.2008.05.002. 